\title{
Magnetic resonance imaging of cervical cancer
}

\author{
Citation for published version (APA):
}

Mongula, J. (2020). Magnetic resonance imaging of cervical cancer: prediction of parametrial invasion and treatment response. [Doctoral Thesis, Maastricht University]. Maastricht University. https://doi.org/10.26481/dis.20201008jm

Document status and date:

Published: 01/01/2020

DOI:

10.26481/dis.20201008jm

Document Version:

Publisher's PDF, also known as Version of record

\section{Please check the document version of this publication:}

- A submitted manuscript is the version of the article upon submission and before peer-review. There can be important differences between the submitted version and the official published version of record.

People interested in the research are advised to contact the author for the final version of the publication, or visit the DOI to the publisher's website.

- The final author version and the galley proof are versions of the publication after peer review.

- The final published version features the final layout of the paper including the volume, issue and page numbers.

Link to publication

\footnotetext{
General rights rights.

- You may freely distribute the URL identifying the publication in the public portal. please follow below link for the End User Agreement:

www.umlib.nl/taverne-license

Take down policy

If you believe that this document breaches copyright please contact us at:

repository@maastrichtuniversity.nl

providing details and we will investigate your claim.
}

Copyright and moral rights for the publications made accessible in the public portal are retained by the authors and/or other copyright owners and it is a condition of accessing publications that users recognise and abide by the legal requirements associated with these

- Users may download and print one copy of any publication from the public portal for the purpose of private study or research.

- You may not further distribute the material or use it for any profit-making activity or commercial gain

If the publication is distributed under the terms of Article $25 \mathrm{fa}$ of the Dutch Copyright Act, indicated by the "Taverne" license above, 


\section{MAGNETIC}

\section{RESONANCE}

IMAGING OF

CERVICAL

CANCER:

PREDICTION OF

PARAMETRIAL INVASION

AND TREATMENT RESPONSE

Jordy Mongula 


\section{MAGNETIC RESONANCE IMAGING OF}

CERVICAL CANCER:

PREDICTION OF PARAMETRIAL INVASION AND TREATMENT RESPONSE 


\section{MAGNETIC RESONANCE IMAGING OF CERVICAL CANCER:}

PREDICTION OF PARAMETRIAL INVASION AND TREATMENT RESPONSE

PROEFSCHRIFT

ISBN: $\quad 978-94-6402-242-1$

Author: J.E. Mongula

Cover design: Ilse Modder | www.ilsemodder.nl

(Cover)Lay-out: Ilse Modder | www.ilsemodder.nl

Printing: Gildeprint Enschede | www.gildeprint.nl

PROEFSCHRIFT
ter verkrijging van de graad van doctor aan de Universiteit Maastricht,
op gezag van Rector Magnificius, Prof. dr. Rianne M. Letschert,
volgens het besluit van het college van Decanen,
in het openbaar te verdedigen op donderdag 8 oktober om 14.00 uur

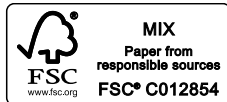

The publication of this thesis was financially supported by:

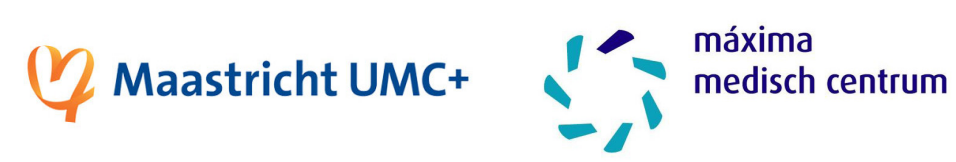

door

Jordy Mongula

Copyright $\odot 2020$ J.E. Mongula.

All rights reserved. No part of this thesis may be reproduced, stored or transmitted in any

form or by any means without the permission of the author. The copyright articles of

the articles that have been published had been transferred to the respective publishers. 


\section{PROMOTOR}

Prof. dr. R.F.P.M. Kruitwagen

\section{COPROMOTORES}

Dr. B.F.M. Slangen

Dr. C. Mihl

\section{BEOORDELINGSCOMMISSIE}

Prof. dr. J.E. Wildberger (voorzitter)

Prof. dr. R. Bekkers

Prof. dr. V. Vandecaveye (Universitair Ziekenhuis Leuven, België)

Dr. R.A. Nout (Erasmus Universitair Medisch Centrum)

Dr. S. Lambrechts

\section{CONTENTS}

Chapter 1

PART I

Chapter 2

Chapter 3

PART II

Chapter 4

Chapter 5

Chapter 6

Chapter 7

Chapter 8

Appendices

\section{MRI in early stage cervical carcinoma, prediction of} parametrial invasion

The additional role of MRI and diffusion weighted imaging for assessment of parametrial invasion of cervical carcinoma. Clin Radiol. 2019;74:790-6

Evaluation of various Apparent Diffusion Coefficient measurement techniques in pre-operative staging of early cervical carcinoma. Eur J Radiol. 2019:118:101-6

MR and PET imaging for predicting response for

locally advanced cervical carcinoma

Predictive criteria for MRI-based evaluation of response

both during and after radiotherapy for cervical cancer. $J$ Contemp Brachytherapy. 2016:8:181-8.

Consecutive magnetic resonance imaging during brachytherapy for cervical carcinoma: predictive value of volume measurements with respect to persistent disease and prognosis. Radiat Oncol. 2015;10:252.

${ }^{18} \mathrm{~F}-\mathrm{FDG}$ Positron emission tomography - Magnetic resonance imaging (PET-MRI) for response assessment after chemo-radiation therapy of cervical carcinoma: an initial experience. EJNMMI Research. 2018;2:8

Summary

Valorisatie

Abbrevations

Publications and presentations

Dankwoord

Curriculum Vitae 


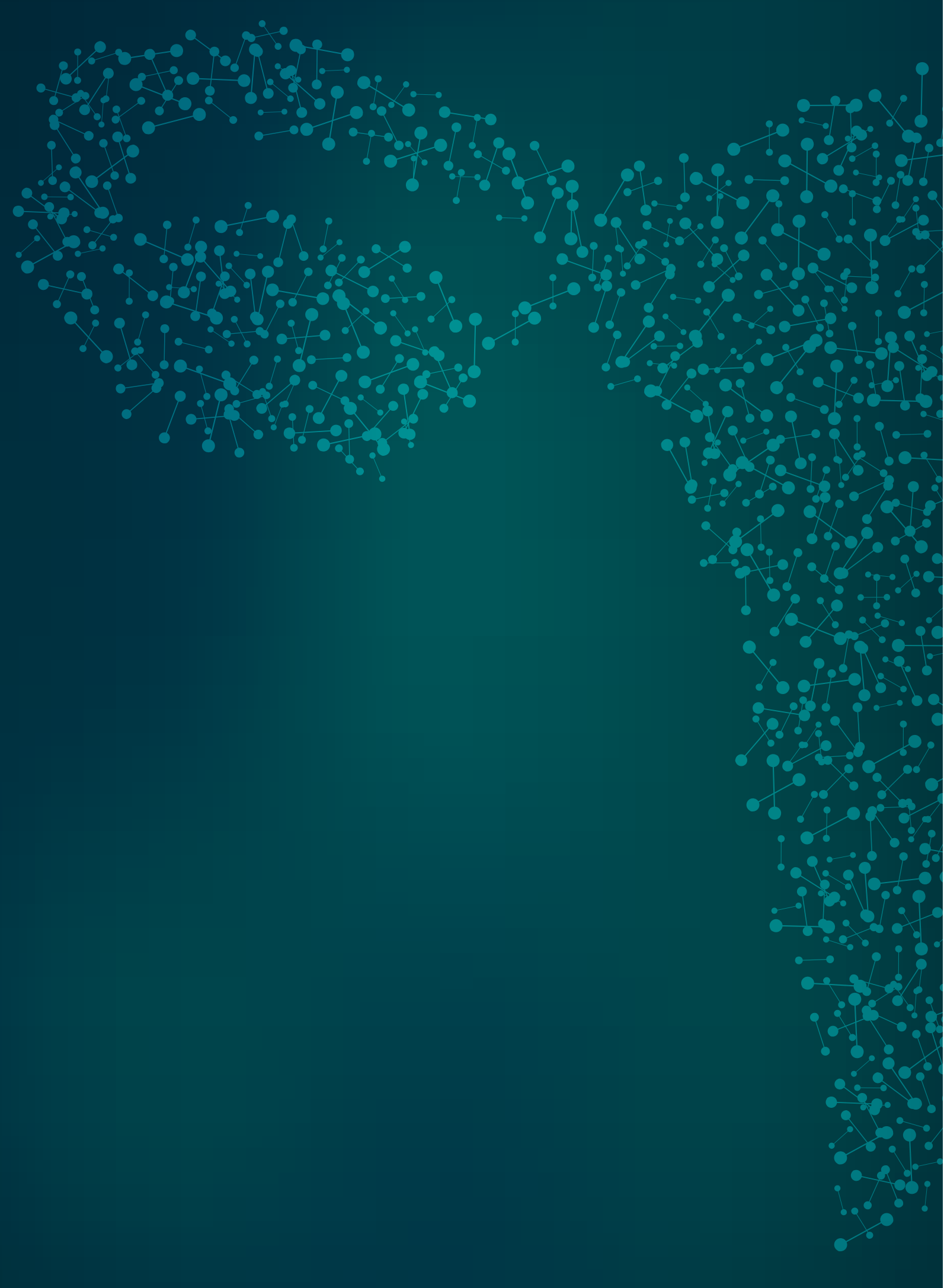

GENERAL INTRODUCTION AND AIMS OF THE THESIS 


\section{CERVICAL CANCER OVERVIEW}

Almost all cervical cancers are caused by persistent infections of high risk types of the human papilloma virus (HPV). ${ }^{(1)}$ HPV hampers normal cell control mechanisms, promotes cell divisions, and prevents apoptosis. ${ }^{(1)}$ Moreover, it has several mechanisms to avoid detection by the immune system. As a consequence, DNA damage is able to accumulate resulting in pre-invasive and eventually invasive cervical cancer. ${ }^{(2)}$

To prevent cervical cancer, primary and secondary prevention are of importance. Primary prevention aims at preventing infection of HPV by vaccination of young female adolescents. In HPV-naive females the bivalent (HPV 16 and 18) vaccine is effective in the prevention of HPV infection in approximately $90 \%$ of the vaccinated females. ${ }^{(1)}$ Moreover, vaccination significantly reduces the risk of pre-invasive cervical cancer lesions both in phase III trials and in population studies. ${ }^{(3,4)}$ Vaccination was implemented for Dutch female adolescents in 2009. Despite a 'catch up' campaign, overall vaccination coverage has plateaued between $46-61 \%{ }^{(5)}$

Secondary prevention aims at detecting pre-invasive cervical lesions; treating highrisk lesions and preventing cervical cancer. HPV-based screening has shown to be superior to cytology based screening. ${ }^{(6)}$ In 2016 HPV-based screening has become standard of practice in the Netherlands, however the participation degree remains low at an average of 57\% (2017). (7) Secondary screening programs are still needed for unvaccinated females including the population born before the start of the HPV vaccination. ${ }^{(8)}$ Also, the currently used vaccines only prevent infection with hrHPV type 16 and 18 , preventing only approximately $70 \%$ of cervical cancers. ${ }^{(2)}$

In spite of extensive screening programs for pre-malignancy, cervical cancer is the third most common malignancy among women worldwide. ${ }^{(9)}$ Without any further interventions, the annual number of women globally affected is expected to rise to 710.000 in 2030. ${ }^{(10)}$

\section{DIAGNOSIS AND STAGING}

Confirmation of the diagnosis of cervical cancer requires a biopsy of the cervix. After a cervical cancer diagnosis, staging provides important information about the extent of cancer in the body and choice of treatment. The main purpose of staging is to provide standard terminology that allows comparison of patients between centers; and to assign patients and their tumors to prognostic groups requiring specific treatments. ${ }^{111}$ For worldwide reproducibility, the staging is based on clinical findings with abdominal, vaginal and rectal examination. ${ }^{(12)}$

TABLE 1. FIGO 2014 stages

I tumor confined to cervix uter

IA1 Microscopic < 3 mm invasion depth

IA2 Microscopic 3-5 $\mathrm{mm}$ invasion depth, $<7 \mathrm{~mm}$ wide

$\mid \mathrm{B} 1<4 \mathrm{~cm}$ diameter

II Extension beyond cervix uter

HA $1<4 \mathrm{~cm}$ diameter with extension in upper $2 / 3$ of the vagin IIA $>4 \mathrm{~cm}$ diameter with extension in upper $2 / 3$ of the vagina IIB Extension in adjacent parametrium

III Extension to pelvic wall or lower vagina IIIA Extension into lower 1/3 of the vagina

IIIB Extension to pelvic side wall or compromising ureter with hydronephrosis

IV Extension to bladder or rectum IVA Extension to mucosa of bladder or rectum IVB Metastases outside the true pelvis Cervical cancer is classified using the FIGO staging systems, which are determined by the International Federation of Gynaecology fiche end of 2018 the FIGO introduced an updated staging system for Cervical Cancer.(11) As all our studies are 


\section{TREATMENT}

Treatment of cervical cancer is tailored to the tumor stage, comorbidities and age, and to the wishes of the patient regarding fertility and management options. The main treatment options are surgery, radiation, or radiation given with chemotherapy (concurrent chemoradiation, CRT). ${ }^{133}$ 14) CRT consists of concurrent platinum-based chemotherapy with external beam radiation therapy and internal brachytherapy. Figure 1 shows the FIGO stages and the modes of treatment.

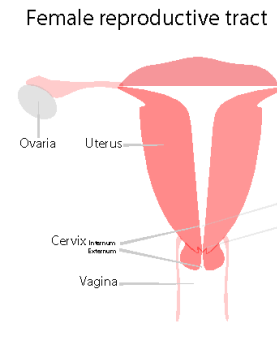

Mode of Treatment

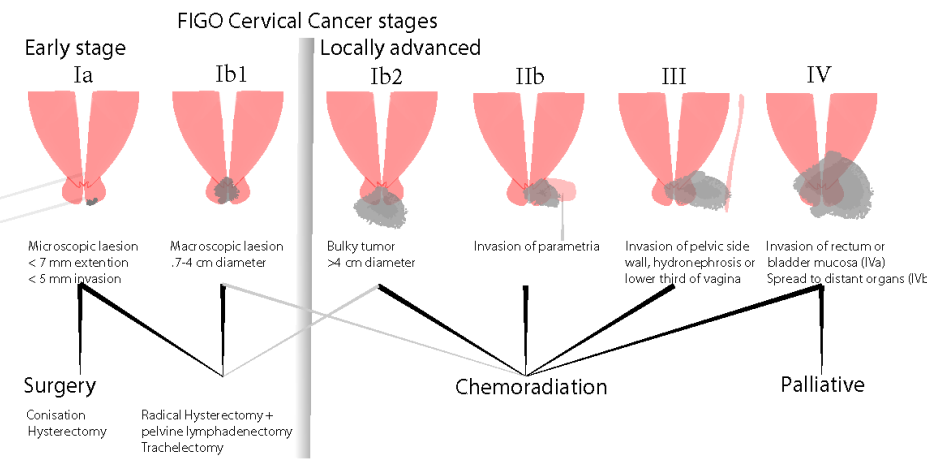

FIGURE 1. Cervical cancer FIGO stages and modes of treatment.

Figo IIA (upper two third of the vagina) is not shown in the figure, treatment depends on the size of the tumor.

\section{FIGO IA}

FIGO stage IA cervical cancer is divided in FIGO stage IA1 and IA2. (table 1). For stage IA1 tumors and FIGO stage IA2 tumors without lymph-vascular space invasion, a conisation or simple hysterectomy is sufficient. Pelvic lymphadenectomy is not advised as lymph node metastasis is less than $1 \%{ }^{(2)}$

For FIGO $1 \mathrm{~A} 2$ tumors with lymph-vascular space invasion, risk of lymph node metastasis is higher and a pelvic lymphadenectomy is advised. Controversy exists whether a simple or radical hysterectomy has to be performed.

\section{FIGO IB1}

Standard treatment consists of radical hysterectomy with pelvic lymphadenectomy or CRT. Factors influencing treatment choice are comorbidity, age and patient preference. In premenopausal patients radiotherapy will lead to menopause and sexual changes. ${ }^{(15)}$
This has to be discussed in counselling. For women with small $(<2 \mathrm{~cm})$ tumors who want to maintain fertility, radical trachelectomy with pelvic lymph node dissection is an option. ${ }^{\left({ }^{16)}\right)}$ In case of unfavourable tumor characteristics after surgery (parametrial invasion, positive resection margins, or a combination of deep stromal invasion, tumor > $4 \mathrm{~cm}$ and/or lymph vascular space invasion), adjuvant RT or even CRT is still advised. ${ }^{(17,18)}$ Also, in case of regional lymph node metastases (15-20\%), adjuvant CRT is advised. This has to be taken into account at counselling, to prevent surgery and adjuvant CRT or RT, with increased long term side effects. ${ }^{(19)}$

\section{FIGO IB2}

For the bulky FIGO IB2 cervical carcinoma, upfront CRT is the preferred treatment as in case of surgery a majority of patients will prove to have unfavourable tumor characteristics and/or lymph node metastases for which adjuvant RT or CRT is recommended. ${ }^{(11)}$ Combining both treatment-modalities results in more morbidity, higher costs and less quality of life with equal survival when compared to CRT alone. ${ }^{19)}$ If lymph node involvement is not expected (or excluded by previous pelvic lymphadenectomy) radical surgery is considered as an option. ${ }^{(11)}$

\section{FIGO IIA}

For FIGO IIA cervical carcinoma treatment mainly depends on the size of the tumor and the possibility to create a 'tumor free' vaginal cuff around the cervical cancer. FIGO IIA1 $<4 \mathrm{~cm}$ is usually treated by surgery. ${ }^{(11)}$ For FIGO IIA2, similar to FIGO IB2 disease, upfront CRT is the preferred treatment as in case of surgery a majority of patients will prove to have unfavourable tumor characteristics and/or lymph node metastases for which adjuvant RT or CRT is recommended. ${ }^{(11)}$

\section{FIGO IIB - IVB}

For the same reasons as in stage IB2, for the higher FIGO stages IIB-IVA upfront CRT is the preferred treatment. ${ }^{(20-22)} \mathrm{As}$ these patients have a higher risk of irradical surgery, surgery alone is unlikely to be curative. (2) The addition of CRT after radical surgery is not recommended due to the risk of (chronic) morbidity. ${ }^{(2)}$ In case of disease spread beyond the pelvis (FIGO IVB), this stage of cervical cancer is usually not considered curable. Palliative treatment options include radiation therapy and/or chemotherapy. 


\section{PROGNOSIS}

The prognosis depends on the FIGO stage (Figure 1, Table 1), combined with unfavourable tumor characteristics (LVI, deep stromal invasion, and large tumor size) and/or the presence of lymph node metastasis. ${ }^{(2,17,18)}$ Lymph node-negative stage IB disease has a 5 year survival of $88 \%$, compared to $73 \%$ for node-positive FIGO IB disease despite additional radiation treatment. ${ }^{(23)}$ Moreover, for FIGO IB1 patients, unfavourable tumor characteristics (LVSI, deep stromal invasion, large tumor size) are associated with a comparable decline in survival, these patients also benefit from additional treatment ${ }^{(17,18)}$ Even worse, in case of postoperative recognition of parametrial invasion, the 5 year survival declines with $20-30 \% .{ }^{(18)}$ This highlights the necessity for additional (C)RT in this group. ${ }^{(18)}$

For locally advanced cervical cancer (FIGO IB2-IVA with the exception of FIGO IIA1), prognosis has significantly increased due to the addition of concurrent platinumbased chemotherapy and the integration of MRI based three-dimensional internal brachytherapy. ${ }^{(13,24)}$ For FIGO IIB overall 3 year survival rate is around $80 \%$, for FIGO III $45 \%$, and FIGO IV 33\%. ${ }^{(24)}$

Despite both CRT and surgical treatments and adjuvant treatments, approximately 10$40 \%$ of FIGO IB-IVA cervical cancer patients will have persistent or recurrent disease. ${ }^{(25-}$

\section{AIMS OF THIS THESIS}

To investigate the accuracy of MRI in staging in early stage cervical cancer, and evaluation of treatment response after CRT.

\section{STAGING IN EARLY STAGE CERVICAL CANCER: PARAMETRIALINVASION}

Accurate staging to allocate patients to the correct treatment and reduce multimodality treatment is essential. Parametrial invasion is the discriminating factor between stage IB and IIB disease in which FIGO IB1 is preferably treated by surgery and FIGO IIB by CRT. (12) Parametrial invasion is traditionally determined by gynaecological examination. Gynaecological examination (palpation of the parametria by recto-vaginal toucher) only has a sensitivity of $40 \% .{ }^{(10)}$ The consequence of this low sensitivity is that $50-60 \%$ of the patients with histologic parametrial invasion will be missed with gynaecological examination. Up to $25 \%$ of these patients, will develop local recurrent disease without additional treatment. As a result, 5-year survival is decreased with 20-30\% ${ }^{(18)}$ Therefore, to improve survival of these patients, adjuvant CRT after surgery is recommended. This will lead to increased morbidity when compared to primary CRT.(18, 29, 30) Moreover, reducing multimodality treatment through more accurate staging is associated with less costs. ${ }^{(19,31)}$

\section{EVALUATION OF TUMOR RESPONSES DURING OR AFTER CRT}

After CRT it is not clear how to determine complete response. ${ }^{(11,32,33)}$ Hysterectomy after CRT for all patients did not improve overall survival, ${ }^{(34)}$ moreover it increased patient morbidity. ${ }^{(35)}$ It has been suggested that salvage surgery for selected patients with residual disease can improve prognosis ${ }^{(32,36)}$, however others could not confirm this. ${ }^{(37)}$ The latter group did acknowledge that their observed lack of benefit was probably due to the inability to select the right patient.

For patients with loco-regional persistent disease after CRT, salvage surgery is an option with a reasonable 5 year survival of $30-40 \% .{ }^{(27,28,38)}$ The survival rate of surgery after CRT is significantly higher when the persistent disease is confined to the cervix. ${ }^{27}$ However, both severe morbidity and mortality $(10 \%)$ of this intervention are high. ${ }^{28,38)}$ Therefore, selection is crucial, also because in case of the simultaneous presence of distant metastases surgery will prove to be futile.

For recurrent disease after surgery or in case of isolated recurrence outside the previous radiation field, CRT is an option to try to salvage these patients. ${ }^{(11)}$ For example, isolated para-aortic lymph node recurrence after CRT has a relatively high response rate up to $78 \% .{ }^{(39)}$

To enable for such a stratification of treatments, it is necessary to accurately evaluate tumor responses during or after CRT, preferable by a non-invasive method. The detection of residual tumor by gynecological examination is limited by false positive and false negative results due to radiation induced fibrosis. Histological biopsies however, are no solution. Sampling errors may explain why small microscopic tumor sites are easily missed, especially when suspected lesions are located outside the cervix or vagina. ${ }^{(32,40)}$ Moreover, the clinical relevance of isolated tumor cells in a biopsy specimen is debatable as the occurrence of microscopic residual disease after completion surgery for CRT shows an almost comparable survival compared to patients with no histological residual disease. ${ }^{(34,41)}$ This can be explained by the ongoing regression of tumor after CRT.

In conclusion, accurate staging to allocate patients to the correct treatment and reduce multimodality treatment is essential. Furthermore, accurate evaluation of tumor 
responses during or after CRT, preferably by a non-invasive method is expected to increase survival while decreasing futile salvage surgery. For both, MRI as a diagnostic tool could be of additional value.

\section{MAGNETIC RESONANCE IMAGING}

In recent years, MRI has increasingly been incorporated in cancer staging, treatment planning and evaluation of response after treatment. MRI is able to portray the distribution of hydrogen nuclei in different tissues in the body by re-emission of the energy from radiofrequency pulses. ${ }^{(42-45)} \mathrm{MRI}$ is able to discriminate between tissues based on their hydrogen content, facilitating the visualization of tissues with normal and pathologic morphology.

T1 weighted (T1W) images depend on the time needed to restore the magnetization of the hydrogen nuclei; they typically show high signal intensity for blood and fat and low intensity for clear fluids. The distinction between different tissues and tumor of T2 weighted (T2W) images depends on the dephasing of the exited hydrogen nuclei. ${ }^{(42)}$ More recently, advanced imaging techniques as diffusion weighted MRI (DWI) have been proposed. ${ }^{(46)} \mathrm{DWI}$ is a technique based on microscopic water diffusion properties. If no cellular boundaries exist water molecules are able to move random (Brownian motion). In vivo boundaries such as tissue cells and cellular membranes hamper free diffusion of water. Increased boundaries by for example malignant tumors with high cellularity will restrict this free diffusion. (Figure $2 A, B$, and $C$ respectively) DWI is able to assess diffusion characteristics of the tissue of interest. ${ }^{(47)}$

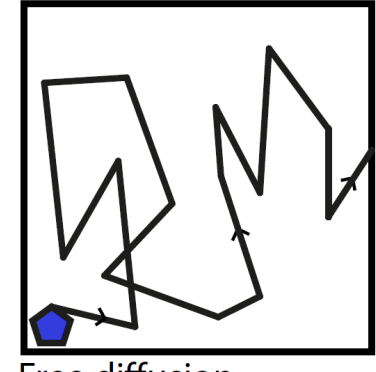

Free diffusion

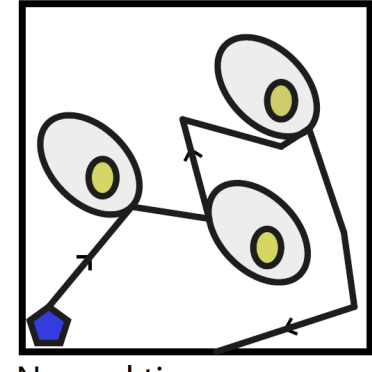

Normal tissue

Normal diffusion

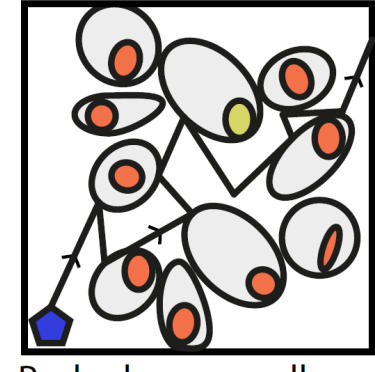

Packed cancer cells Restricted diffusion
FIGURE 2. Principle of diffusion weighted imaging

DWI is obtained by two opposing symmetrical T2W gradients. The B-value corresponds with the timeframe between these opposing gradients reflecting the time for mobilization of water. Mobilized water will not return to its resting state after the 
opposing gradient, showing low signal intensity on the DW-images. In particular, the tissue of interest such as malignant tumors tend to increase their cellularity, this is accompanied by a limited movement of water and a high signal intensity on the DWimages. ${ }^{(48)}$ Quantitative assessment of DWI is performed with the Apparent Diffusion Coefficient (ADC). From the generated diffusion images with different $B$-values, an ADC map is generated. The ADC is diffusion measured within a voxel; a lower ADC reflects more diffusion restriction and therefore tissue cellularity and membrane integrity. ${ }^{(49)}$

\section{MAGNETIC RESONANCE IMAGING IN CERVICAL CANCER - STAGING}

MRI has the potential to assess the local extent of a cervical tumor due to its excellent soft tissue contrast. ${ }^{(50)} \mathrm{T} 2 \mathrm{~W}$ sequences are the preferred sequences to detect cervical cancer both before and after treatment as cervical cancer has a higher signal intensity compared to normal cervical stroma and other adjacent tissue. ${ }^{(43,51)} \mathrm{T} 2 \mathrm{~W}$ sequences are also used for MRI guided brachytherapy, applicator based distortions and artefacts are minimal for $\mathrm{T} 2 \mathrm{~W}$ sequences. ${ }^{(52)} \mathrm{T} 1 \mathrm{~W}$ sequences are primarily used to assess possible lymph node involvement.

Recently, the updated FIGO guideline (2018) supports the use of MRI for the staging of cervical cancer. Until now, staging was based on clinical examination. ${ }^{(12)}$ A recent systematic review compared clinical examination with MRI in cervical cancer. ${ }^{(53)}$ The latter proved to be superior to clinical examination for the evaluation of parametrial invasion and for the evaluation of advanced disease. MRI had a pooled sensitivity of $84 \%$, compared to clinical examination having a sensitivity of only $40 \%$ for the evaluation of parametrial invasion, with comparable specificity $(92-93 \%) .{ }^{(53,54)}$ However, regardless of a more strict selection, $13-25 \%$ of parametrial invasion is missed, even with MRI. ${ }^{(53,55)}$

On MRI, absence of parametrial invasion is most often illustrated by a regular hypointense cervical stromal ring, without signs of disruption. ${ }^{(56,57)}$ When the cervical stromal ring is disrupted, the difference between a subtle invading cervical tumor and pressure of the mass with a thin boundary and/or biological reaction in the adjacent parametrium without parametrium invasion is difficult. Therefore, due to tumor growth, pressure, edema and the biological reaction of the surrounding cervical tissue, assessing parametrial invasion on conventional T2W MRI is prone to false positive results up to $50 \%{ }^{\left({ }^{(4)}\right)} \mathrm{DWI}$ bears the potential to overcome this problem. The biological reaction of the adjacent tissue is not expected to show diffusion restriction in contrast to malignant cervical cancer. ${ }^{(58)}$
The potential of DWI for the evaluation of parametrial invasion has been highlighted in two retrospective single institution trials. ${ }^{(59,60)}$ Two prospective trials showed some potential of DWI for the evaluation of parametrial invasion however due to the small (sub)set of patients with DWI they could not draw definitive conclusions. ${ }^{(50,61,62)}$

The main focus of DWI in cervical cancer has been on assessing its derived quantitative parameter ADC. The ADC is correlated with pathologic features as tissue cellularity, the differentiation grade of the tumor and possible LVSI. ${ }^{(63-65)}$ Therefore, ADC could correlate with the malignant potential of the tumor. To date, studies on ADC showed a lower $A D C(<0.85-0.90)$ to correlate with a higher chance of parametrial involvement. ${ }^{(66,67)}$ The results for assessing other risk factors for disease recurrence such as LVSI and lymph node metastases are still conflicting ${ }^{(67,68)}$ But more importantly, mean ADC correlates with risk of recurrence and survival in three retrospective studies. $\left.{ }^{67,}, 69,70\right)$ However, multiple authors admit there are limitations in identifying the Region Of Interest (ROI) for measuring the ADC. ${ }^{(46,66)}$ As a consequence corresponding cut-off values for the mentioned risk factors are not clear. Methods for measuring ADC are not uniform, in most studies ADC is depicted by a single or multiple small ROI's. ${ }^{(66,67)}$ The need to select the appropriate $\mathrm{ROI}$ and the different types of ROIs induce a variability between and within studies. Therefore, these methods could be inferior compared to total tumor analyses as tumor heterogeneity and therefore possible tumor aggressiveness might be underestimated. ${ }^{(71)}$ To date, it remains unclear if three dimensional total tumor ADC is superior to two dimensional single ROI ADC measurements.

\section{MAGNETIC RESONANCE IMAGING IN ADVANCED CERVICAL CANCER - EVALUATION OF TREATMENT RESPONSE}

MRI is often used to assess pelvic and cervical tumors after radiation. $(33,40,57,72,73)$ However, it is difficult to assess MRI after radiation. The irradiated tumor bed is often replaced by fibrotic tissue; differentiating fibrotic from residual tumor tissue is difficult. ${ }^{(33,}$ ${ }^{72,74)}$ Conventional T2W sequences lack the power to adequately differentiate between radiation induced changes and active residual tumor after CRT. Both are able to show isointense signal intensity on T2W MRI imaging. ${ }^{(33)}$ Besides substantial uncertainty (up to $35 \%$ ) due to radiation induced changes, a rather low specificity and high false positive rate is reported. ${ }^{(33,40)} \mathrm{MRI}$ is also performed to facilitate the treatment planning of the CRT. ${ }^{(20-22)}$ As a result, this MR guided (brachy) radiation therapy leads to individual patient treatment with more accurate dose administration to the target volume, increasing local complete response. ${ }^{(21,24)}$ Tumor volume assessment on repeated MRIs during radiotherapy treatment can be used to predict tumor response to radiotherapy. ${ }^{(75-78)}$ Tumor volumetry for predicting treatment response on MRI with 


\section{GENERAL AIMS}

\section{POSITRON EMISSION TOMOGRAPHY IN ADVANCED CERVICAL CANCER -}

\section{EVALUATION OF TREATMENT RESPONSE}

In recent years Fluorodeoxyglucose-positron emission tomography (FDG-PET) has increasingly been incorporated in cancer staging, treatment planning and after treatment. PET is based on the potential of reflecting metabolic and proliferative activity of tumor cells by using radioactive labeled molecules, mostly 18-fluorodeoxyglucose (18F-FDG). This glucose derivate reflects malignant metabolic activity due to upregulation of GLUT receptors and glucose use by tumor cells, the intracellular concentration of FDG-6-phosphate reflects metabolic activity.

FDG-PET might overcome the false positive rate of conventional T2W MRI for residual tumor after radiation. FDG-PET has shown to be able to predict tumor response and metastasis after treatment. ${ }^{(39,79,80)}$ In general, FDG-PET is combined with low-dose computed tomography (CT) images. The spatial resolution of these CT images is rather poor as compared to MRI. As a consequence, if local treatment failure is suspected based on PET-CT, additional MRI is necessary for adequate treatment planning. ${ }^{.81}$

The combination of the MRI with functional FDG-PET imaging could reduce false positive results of MRI by allowing for differentiation between residual tumor and radiation induced fibrotic tissue. ${ }^{(82,83)}$ FDG uptake is not expected in fibrotic tissue in comparison to residual tumor.

\section{PART 1}

1. To evaluate the diagnostic performance of DWI in addition to regular T2W sequences for the assessment of parametrial invasion.

2. To compare different $A D C$ measurement techniques and to evaluate mean $A D C$ as a parameter for predicting parametrial and lymph node involvement

\section{PART 2}

3. To evaluate the ability of pelvic MRI to detect residual tumor after radiation and to identify imaging criteria predictive for residual tumor.

4. To evaluate tumor volume (regression) during radiation to discriminate between patients with a complete response and those with treatment failure.

5. To assess the ability of PET-MRI for the evaluation of treatment response. 


\section{REFERENCE}

1. Crosbie EJ, Einstein MH, Franceschi S, Kitchener HC. Human papillomavirus and cervical cancer. Lancet. 2013;382(9895):889-99.

2. Cohen PA, Jhingran A, Oaknin A, Denny L. Cervical cancer. Lancet. 2019;393(10167):169-82.

3. Group FIS. Quadrivalent vaccine against human papillomavirus to prevent high-grade cervical lesions. N Engl J Med. 2007;356(19):1915-27

4. Brotherton JM, Fridman M, May CL, Chappell G, Saville AM, Gertig DM. Early effect of the HPV vaccination programme on cervical abnormalities in Victoria, Australia: an ecological study. Lancet. 2011;377(9783):2085-

5. RIVM. Vaccinatiegraad en jaarverslag Rijksvaccinatieprogramma Nederland 2018. https://wwwrivmnl/ bibliotheek/rapporten/2019-0015pdf.

6. Ronco G, Giorgi-Rossi P, Carozzi F, Confortini M, Dalla Palma P, Del Mistro A, et al. Efficacy of human papillomavirus testing for the detection of invasive cervical cancers and cervical intraepithelial neoplasia: a randomised controlled trial. Lancet Oncol. 2010;11(3):249-57.

7. RIVM. Factsheet Bevolkingsonderzoek baarmoederhalskanker in 2018. https://wwwrivmnl/sites/default/ files/2019-08/Factsheet\%20bevolkingsonderzoek\%20baarmoederhalskanker\%202018pdf. 2017.

8. Qendri V, Schurink-Van 't Klooster TM, Bogaards JA, Berkhof J. Ten years of HPV vaccination in the Netherlands: current evidence and future challenges in HPV-related disease prevention. Expert Rev Vaccines. 2018:17(12):1093-104

9. Ogilvie G, Nakisige C, Huh WK, Mehrotra R, Franco EL, Jeronimo J. Optimizing secondary prevention of cervical cancer: Recent advances and future challenges. Int J Gynaecol Obstet. 2017;138 Suppl 1:15-9.

10. Ginsburg O, Bray F, Coleman MP, Vanderpuye V, Eniu A, Kotha SR, et al. The global burden of women's cancers: a grand challenge in global health. Lancet. 2017;389(10071):847-60

11. Cibula D, Potter R, Planchamp F, Avall-Lundqvist E, Fischerova D, Haie Meder C, et al. The European Society of Gynaecological Oncology/European Society for Radiotherapy and Oncology/European Society of Pathology guidelines for the management of patients with cervical cancer. Radiother Oncol. 2018;127(3):404-16.

12. Oncology FCoG. FIGO staging for carcinoma of the vulva, cervix, and corpus uteri. Int J Gynaecol Obstet. 2014;:125(2):97-8

13. Green J, Kirwan J, Tierney J, Vale C, Symonds P, Fresco L, et al. Concomitant chemotherapy and radiation therapy for cancer of the uterine cervix. Cochrane Database Syst Rev. 2005(3):CD002225.

14. Salicru SR, de la Torre JF, Gil-Moreno A. The surgical management of early-stage cervical cancer. Curr Opin Obstet Gynecol. 2013;25(4):312-9.

15. Donovan KA, Taliaferro LA, Alvarez EM, Jacobsen PB, Roetzheim RG, Wenham RM. Sexual health in women treated for cervical cancer: characteristics and correlates. Gynecologic oncology. 2007:104(2):428-34.

16. Plante M, Gregoire J, Renaud MC, Roy M. The vaginal radical trachelectomy: an update of a series of 125 cases and 106 pregnancies. Gynecol Oncol. 2011;121(2):290-7.
17. Sedlis A, Bundy BN, Rotman MZ, Lentz SS, Muderspach LI, Zaino RJ. A randomized trial of pelvic radiation therapy versus no further therapy in selected patients with stage IB carcinoma of the cervix after radical hysterectomy and pelvic lymphadenectomy: A Gynecologic Oncology Group Study. Gynecol Oncol. 1999;73(2):177-83

18. Peters WA, 3rd, Liu PY, Barrett RJ, 2nd, Stock RJ, Monk BJ, Berek JS, et al. Concurrent chemotherapy and pelvic radiation therapy compared with pelvic radiation therapy alone as adjuvant therapy after radical surgery in high-risk early-stage cancer of the cervix. J Clin Oncol. 2000;18(8):1606-13.

19. Katanyoo K, Praditsitthikorn N, Tangjitgamol S, Manusirivithaya S, Supawattanabodee B. Cost-utility analysis of treatments for stage IB cervical cancer. J Gynecol Oncol. 2014;25(2):97-104.

20. Eifel PJ, Winter K, Morris M, Levenback C, Grigsby PW, Cooper J, et al. Pelvic irradiation with concurrent chemotherapy versus pelvic and para-aortic irradiation for high-risk cervical cancer: an update of radiation therapy oncology group trial (RTOG) 90-01. J Clin Oncol. 2004;22(5):872-80.

21. Harkenrider MM, Alite F, Silva SR, Small W, Jr. Image-Based Brachytherapy for the Treatment of Cervical Cancer. Int J Radiat Oncol Biol Phys. 2015:92(4):921-34.

22. Sturdza A, Potter R, Fokdal LU, Haie-Meder C, Tan LT, Mazeron R, et al. Image guided brachytherapy in locally advanced cervical cancer: Improved pelvic control and survival in RetroEMBRACE, a multicenter cohort study. Radiother Oncol. 2016;120(3):428-33.

23. Lee $\mathrm{YN}$, Wang $\mathrm{KL}$, Lin $\mathrm{MH}$, Liu CH, Wang KG, Lan CC, et al. Radical hysterectomy with pelvic lymph node dissection for treatment of cervical cancer: a clinical review of 954 cases. Gynecol Oncol. 1989;32(2):135-

24. Potter R, Georg P, Dimopoulos JC, Grimm M, Berger D, Nesvacil N, et al. Clinical outcome of protocol based image (MRI) guided adaptive brachytherapy combined with 3D conformal radiotherapy with or without chemotherapy in patients with locally advanced cervical cancer. Radiotherapy and oncology : journal of the European Society for Therapeutic Radiology and Oncology. 2011:100(1):116-23.

25. Elit L, Fyles AW, Devries MC, Oliver TK, Fung-Kee-Fung M, Gynecology Cancer Disease Site G. Follow-up or women after treatment for cervical cancer: a systematic review. Gynecol Oncol. 2009:114(3):528-35.

26. Fagundes H, Perez CA, Grigsby PW, Lockett MA. Distant metastases after irradiation alone in carcinoma of the uterine cervix. Int J Radiat Oncol Biol Phys. 1992;24(2):197-204.

27. Hong JH, Tsai CS, Lai CH, Chang TC, Wang CC, Chou HH, et al. Recurrent squamous cell carcinoma of cervix after definitive radiotherapy. Int J Radiat Oncol Biol Phys. 2004;60(1):249-57.

28. Kim TH, Kim MH, Kim BJ, Park SI, Ryu SY, Cho CK. Prognostic Importance of the Site of Recurrence in Patients With Metastatic Recurrent Cervical Cancer. Int J Radiat Oncol Biol Phys. 2017;98(5):1124-31.

29. Landoni F, Colombo A, Milani R, Placa F, Zanagnolo V, Mangioni C. Randomized study between radical surgery and radiotherapy for the treatment of stage IB-IIA cervical cancer: 20-year update. J Gynecol Oncol. 2017;28(3):e34

30. Landoni F, Maneo A, Colombo A, Placa F, Milani R, Perego P, et al. Randomised study of radical surgery versus radiotherapy for stage Ib-Ila cervical cancer. Lancet. 1997;350(9077):535-40

31. Pandharipande PV, Choy G, del Carmen MG, Gazelle GS, Russell AH, Lee SI. MRI and PET/CT for triaging 
stage IB clinically operable cervical cancer to appropriate therapy: decision analysis to assess patient outcomes. AJR Am J Roentgenol. 2009;192(3):802-14.

32. Nijhuis ER, van der Zee AG, in 't Hout BA, Boomgaard JJ, de Hullu JA, Pras E, et al. Gynecologic examination and cervical biopsies after (chemo) radiation for cervical cancer to identify patients eligible for salvage surgery. International journal of radiation oncology, biology, physics. 2006;66(3):699-705

33. Vincens E, Balleyguier C, Rey A, Uzan C, Zareski E, Gouy S, et al. Accuracy of magnetic resonance imaging in predicting residual disease in patients treated for stage IB2/II cervical carcinoma with chemoradiation therapy : correlation of radiologic findings with surgicopathologic results. Cancer. 2008;113(8):2158-65.

34. Keys HM, Bundy BN, Stehman FB, Okagaki T, Gallup DG, Burnett AF, et al. Radiation therapy with and without extrafascial hysterectomy for bulky stage IB cervical carcinoma: a randomized trial of the Gynecologic Oncology Group. Gynecol Oncol. 2003;89(3):343-53.

35. Touboul C, Uzan C, Mauguen A, Gouy S, Rey A, Pautier P, et al. Prognostic factors and morbidities after completion surgery in patients undergoing initial chemoradiation therapy for locally advanced cervical cancer. Oncologist. 2010;15(4):405-15

36. Houvenaeghel G, Lelievre L, Buttarelli M, Jacquemier J, Carcopino X, Viens P, et al. Contribution of surgery in patients with bulky residual disease after chemoradiation for advanced cervical carcinoma. Eur J Surg Oncol. 2007;33(4):498-503

37. Gosset M, Chargari C, Bentivegna E, Leary A, Genestie C, Maulard A, et al. Should We Cease to Perform Salvage Hysterectomy After Chemoradiation and Brachytherapy in Locally Advanced Cervical Cancer? Anticancer Res. 2019:39(6):2919-26

38. Peiretti M, Zapardiel I, Zanagnolo V, Landoni F, Morrow CP, Maggioni A. Management of recurrent cervical cancer: a review of the literature. Surg Oncol. 2012;21(2)::59-66.

39. Siva S, Deb S, Young RJ, Hicks RJ, Callahan J, Bressel M, et al. (1)(8)F-FDG PET/CT following chemoradiation of uterine cervix cancer provides powerful prognostic stratification independent of HPV status: a prospective cohort of 105 women with mature survival data. Eur J Nucl Med Mol Imaging. 2015;42(12):1825-32.

40. Hatano K, Sekiya Y, Araki H, Sakai M, Togawa T, Narita Y, et al. Evaluation of the therapeutic effect of radiotherapy on cervical cancer using magnetic resonance imaging. International journal of radiation oncology, biology, physics. 1999;45(3):639-44

41. Ferrandina G, Margariti PA, Smaniotto D, Petrillo M, Salerno MG, Fagotti A, et al. Long-term analysis of clinical outcome and complications in locally advanced cervical cancer patients administered concomitant chemoradiation followed by radical surgery. Gynecol Oncol. 2010;119(3):404-10.

42. Armstrong P, Keevil SF. Magnetic resonance imaging--1: Basic principles of image production. BMJ. 1991;303(6793):35-40.

43. Follen M, Levenback CF, lyer RB, Grigsby PW, Boss EA, Delpassand ES, et al. Imaging in cervical cancer. Cancer. 2003:98(9 Suppl):2028-38.

44. Bragg DG, Hricak H. Imaging in gynecologic malignancies. Cancer. 1993;71(4 Suppl):1648-51.

45. Zand KR, Reinhold C, Abe H, Maheshwari S, Mohamed A, Upegui D. Magnetic resonance imaging of the cervix. Cancer Imaging. 2007:7:69-76.
46. Harry VN, Semple SI, Parkin DE, Gilbert FJ. Use of new imaging techniques to predict tumour response to therapy. Lancet Oncol. 2010;11(1):92-102.

47. Qayyum A. Diffusion-weighted imaging in the abdomen and pelvis: concepts and applications. Radiographics. 2009:29(6):1797-810

48. Surov A, Meyer HJ, Wienke A. Correlation between apparent diffusion coefficient (ADC) and cellularity is different in several tumors: a meta-analysis. Oncotarget. 2017;8(35):59492-9.

49. Sala E, Rockall A, Rangarajan D, Kubik-Huch RA. The role of dynamic contrast-enhanced and diffusion weighted magnetic resonance imaging in the female pelvis. Eur J Radiol. 2010;76(3):367-85.

50. Balleyguier C, Sala E, Da Cunha T, Bergman A, Brkljacic B, Danza F, et al. Staging of uterine cervical cancer with MRI: guidelines of the European Society of Urogenital Radiology. Eur Radiol. 2011;21(5):1102-10.

51. Sala E, Rockall AG, Freeman SJ, Mitchell DG, Reinhold C. The added role of MR imaging in treatment stratification of patients with gynecologic malignancies: what the radiologist needs to know. Radiology. 2013;266(3):717-40

52. Beddy P, Rangarajan RD, Sala E. Role of MRI in intracavitary brachytherapy for cervical cancer: what the radiologist needs to know. AJR Am J Roentgenol. 2011;196(3):W341-7.

53. Thomeer MG, Gerestein C, Spronk S, van Doorn HC, van der Ham E, Hunink MG. Clinical examination versus magnetic resonance imaging in the pretreatment staging of cervical carcinoma: systematic review and meta-analysis. Eur Radiol. 2013:23(7):2005-18

54. Jolly S US, Bhatla N, Johnston C, Maturen K. Improving Global Outcomes in Cervical Cancer: The Time Has Come for International Federation of Gynecology and Obstetrics Staging to Formally Incorporate Advanced Imaging. Journal of Global Oncology. 2017:1-6.

55. Kato T, Takashima A, Kasamatsu T, Nakamura K, Mizusawa J, Nakanishi T, et al. Clinical tumor diameter and prognosis of patients with FIGO stage IB1 cervical cancer (JCOG0806-A). Gynecol Oncol. 2015;137(1):34-9.

56. Shweel MA, Abdel-Gawad EA, Abdel-Gawad EA, Abdelghany HS, Abdel-Rahman AM, Ibrahim EM. Uterine cervical malignancy: diagnostic accuracy of MRI with histopathologic correlation. J Clin Imaging Sci. 2012;2:42.

57. Hricak H, Lacey CG, Sandles LG, Chang YC, Winkler ML, Stern JL. Invasive cervical carcinoma: comparison of MR imaging and surgical findings. Radiology. 1988;166(3):623-31

58. Charles-Edwards EM, Messiou C, Morgan VA, De Silva SS, McWhinney NA, Katesmark M, et al. Diffusionweighted imaging in cervical cancer with an endovaginal technique: potential value for improving tumor detection in stage la and Ib1 disease. Radiology. 2008;249(2):541-50.

59. Park JJ, Kim CK, Park SY, Park BK. Parametrial invasion in cervical cancer: fused T2-weighted imaging and high-b-value diffusion-weighted imaging with background body signal suppression at $3 \mathrm{~T}$. Radiology. 2015;274(3):734-41.

60. Qu JR, Qin L, Li X, Luo JP, Li J, Zhang HK, et al. Predicting Parametrial Invasion in Cervical Carcinoma (Stages IB1, IB2, and IIA): Diagnostic Accuracy of T2-Weighted Imaging Combined With DWI at 3 T. AJR Am J Roentgenol. 2018;210(3):677-84

61. Exner M, Kuhn A, Stumpp P, Hockel M, Horn LC, Kahn T, et al. Value of diffusion-weighted MRI in diagnosis 
of uterine cervical cancer: a prospective study evaluating the benefits of DWI compared to conventional MR sequences in a 3 T environment. Acta Radiol. 2016;57(7):869-77.

62. Bourgioti C, Chatoupis K, Rodolakis A, Antoniou A, Tzavara C, Koutoulidis V, et al. Incremental prognostic value of MRI in the staging of early cervical cancer: a prospective study and review of the literature. Clin Imaging. 2016:40(1):72-8.

63. Payne GS, Schmidt M, Morgan VA, Giles S, Bridges J, Ind T, et al. Evaluation of magnetic resonance diffusion and spectroscopy measurements as predictive biomarkers in stage 1 cervical cancer. Gynecol Oncol. 2010;116(2):246-52.

64. Downey K, Riches SF, Morgan VA, Giles SL, Attygalle AD, Ind TE, et al. Relationship between imaging biomarkers of stage I cervical cancer and poor-prognosis histologic features: quantitative histogram analysis of diffusion-weighted MR images. AJR Am J Roentgenol. 2013;200(2):314-20.

65. Karunya RJ, Tharani P, John S, Kumar RM, Das S. Role of Functional Magnetic Resonance Imaging Derived Parameters as Imaging Biomarkers and Correlation with Clinicopathological Features in Carcinoma of Uterine Cervix. J Clin Diagn Res. 2017;11(8):XC06-XC11.

66. Park JJ, Kim CK, Park SY, Park BK, Kim B. Value of diffusion-weighted imaging in predicting parametrial invasion in stage IA2-IIA cervical cancer. Eur Radiol. 2014;24(5):1081-8.

67. Nakamura K, Joja I, Nagasaka T, Fukushima C, Kusumoto T, Seki N, et al. The mean apparent diffusion coefficient value (ADCmean) on primary cervical cancer is a predictive marker for disease recurrence. Gynecol Oncol. 2012;127(3):478-83

68. Kuang F, Ren J, Zhong Q, Liyuan F, Huan Y, Chen Z. The value of apparent diffusion coefficient in the assessment of cervical cancer. Eur Radiol. 2013;23(4):1050-8

69. Zhou G, Chen X, Tang F, Zhou J, Wang Y, Wang Z. The Value of Diffusion-Weighted Imaging in Predicting the Prognosis of Stage IB-IIA Cervical Squamous Cell Carcinoma After Radical Hysterectomy. Int J Gynecol Cancer. 2016;26(2):361-6.

70. Micco M, Vargas HA, Burger IA, Kollmeier MA, Goldman DA, Park KJ, et al. Combined pre-treatment MRI and 18F-FDG PET/CT parameters as prognostic biomarkers in patients with cervical cancer. Eur J Radiol. 2014:83(7):1169-76.

71. Schob S, Meyer HJ, Pazaitis N, Schramm D, Bremicker K, Exner M, et al. ADC Histogram Analysis of Cervical Cancer Aids Detecting Lymphatic Metastases-a Preliminary Study. Mol Imaging Biol. 2017.

72. Dresen RC, Beets GL, Rutten HJ, Engelen SM, Lahaye MJ, Vliegen RF, et al. Locally advanced rectal cancer MR imaging for restaging after neoadjuvant radiation therapy with concomitant chemotherapy. Part I. Are we able to predict tumor confined to the rectal wall? Radiology. 2009;252(1):71-80

73. Manfredi R, Maresca G, Smaniotto D, Greggi S, Andrulli D, Rabitti C, et al. Cervical cancer response to neoadjuvant therapy: MR imaging assessment. Radiology. 1998;209(3):819-24.

74. Vliegen RF, Beets GL, Lammering $G$, Dresen RC, Rutten $H J$, Kessels $A G$, et al. Mesorectal fascia invasion after neoadjuvant chemotherapy and radiation therapy for locally advanced rectal cancer: accuracy of MR imaging for prediction. Radiology. 2008;246(2):454-62.

75. Mayr NA, Magnotta VA, Ehrhardt JC, Wheeler JA, Sorosky JI, Wen BC, et al. Usefulness of tumor volumetry by magnetic resonance imaging in assessing response to radiation therapy in carcinoma of the uterine cervix. Int J Radiat Oncol Biol Phys. 1996;35(5):915-24

76. Mayr NA, Wang JZ, Lo SS, Zhang D, Grecula JC, Lu L, et al. Translating response during therapy into ultimate treatment outcome: a personalized 4-dimensional MRI tumor volumetric regression approach in cervical cancer. Int J Radiat Oncol Biol Phys. 2010;76(3):719-27.

77. Wang JZ, Mayr NA, Zhang D, Li K, Grecula JC, Montebello JF, et al. Sequential magnetic resonance imaging of cervical cancer: the predictive value of absolute tumor volume and regression ratio measured before. during, and after radiation therapy. Cancer. 2010;116(21):5093-101.

78. Nam H, Park W, Huh SJ, Bae DS, Kim BG, Lee JH, et al. The prognostic significance of tumor volume regression during radiotherapy and concurrent chemoradiotherapy for cervical cancer using MRI. Gynecol Oncol. 2007:107(2):320-5,

79. Schwarz JK, Siegel BA, Dehdashti F, Grigsby PW. Association of posttherapy positron emission tomography with tumor response and survival in cervical carcinoma. JAMA. 2007;298(19):2289-95.

80. Choi J, Kim HJ, Jeong YH, Lee JH, Cho A, Yun M, et al. The Role of (18) F-FDG PET/CT in Assessing Therapy Response in Cervix Cancer after Concurrent Chemoradiation Therapy. Nucl Med Mol Imaging. 2014:48(2):130-6.

81. Lee SI, Catalano OA, Dehdashti F. Evaluation of gynecologic cancer with MR imaging, 18F-FDG PET/CT, and PET/MR imaging. J Nucl Med. 2015;56(3):436-43.

82. Grueneisen J, Beiderwellen K, Heusch P, Gratz M, Schulze-Hagen A, Heubner M, et al. Simultaneous positron emission tomography/magnetic resonance imaging for whole-body staging in patients with recurrent gynecological malignancies of the pelvis: a comparison to whole-body magnetic resonance imaging alone. Invest Radiol. 2014;49(12):808-15

83. Beiderwellen K, Grueneisen J, Ruhlmann V, Buderath P, Aktas B, Heusch P, et al. [(18)F]FDG PET/MRI vs. PET/ CT for whole-body staging in patients with recurrent malignancies of the female pelvis: initial results. Eur J Nucl Med Mol Imaging. 2015;42(1):56-65. 


\section{PART I}

MRI IN EARLY STAGE CERVICAL CARCINOMA, PREDICTION OF PARAMETRIAL INVASION. 


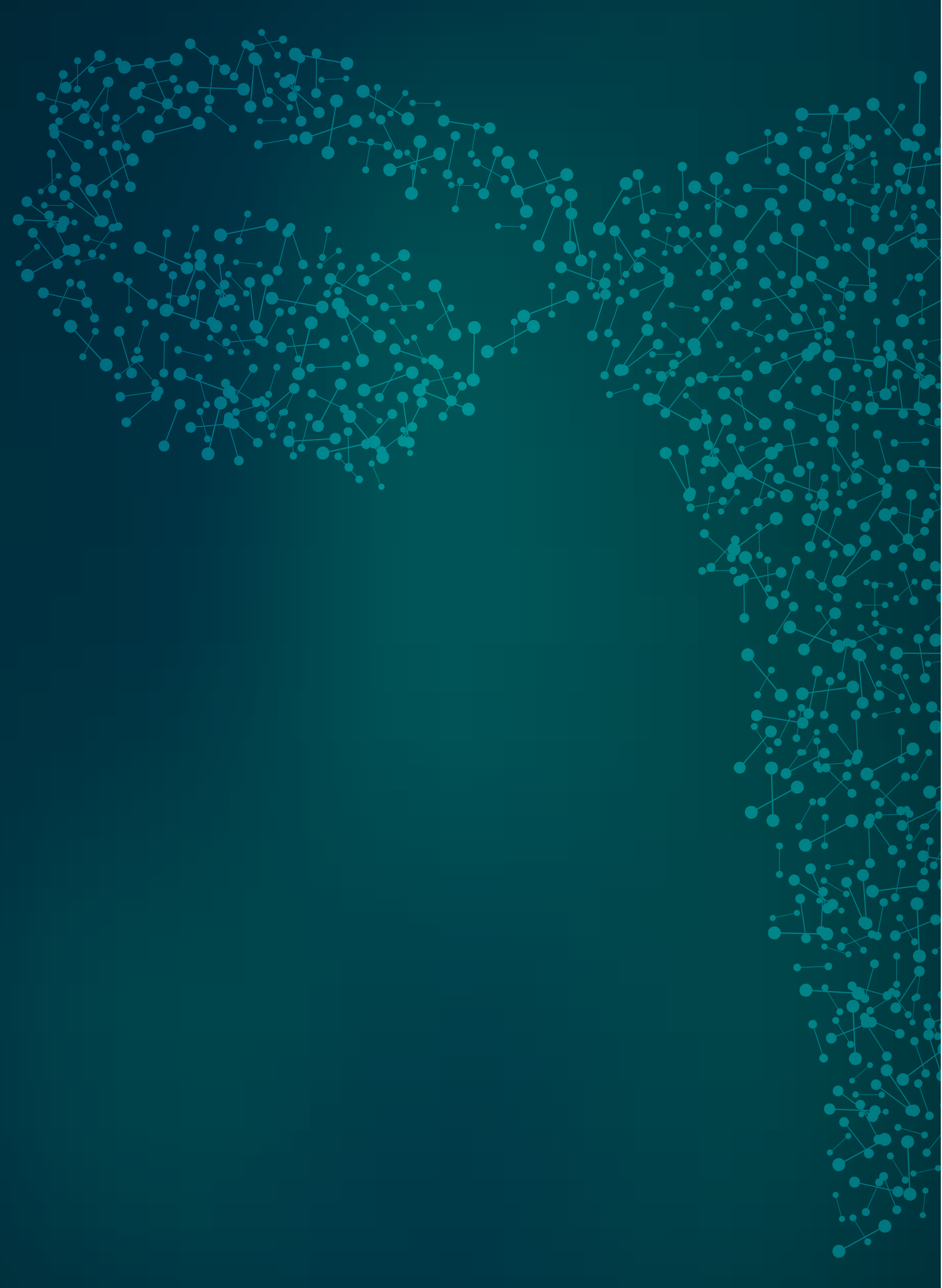

THE ADDITIONAL ROLE OF MRI AND DIFFUSION WEIGHTED IMAGING FOR ASSESSMENT OF PARAMETRIAL INVASION OF CERVICAL CARCINOMA.

Mongula JE Bakers FCH

Mihl C

van Gorp T

Kruitwagen RFPM

Slangen BFM 


\section{ABSTRACT}

\section{OBJECTIVES}

The treatment of cervical cancer is dependent on stage. MRI is increasingly being used to optimize staging to allocate cervical cancer patients to the correct treatment. This study aims to evaluate the diagnostic performance of diffusion weighted MRI (DWI), with and without fusion images, in addition to regular T2-weighted (T2W) sequences for assessment of parametrial invasion.

\section{METHODS}

This prospective cohort included cervical cancer patients who underwent preoperative MRI with T2W and axial DWI sequences prior to radical hysterectomy. Retrospectively, two radiologists independently and blindly scored the likelihood of parametrial invasion by means of a 6 -point confidence scale. Parametrial invasion was determined by surgical-pathologic results. Performance indices for diagnostic tests and receiveroperating-characteristics curve analyses (AUC) were performed. P-values $<0.05$ were considered statistical significant. Ethical board approval was obtained.

\section{RESULTS}

Of 65 included patients, parametrial invasion was found in 8 patients. A statistically significant increase in diagnostic performance for the assessment of parametrial invasion was found when T2W-MRI was fused with DWI (Fusion T2W/DWI), especially decreasing false positive findings: The positive predictive value of parametrial invasion using T2WMRI vs Fusion T2W MRI/DWI increased from 29\% to 50\% in observer 1 and from 23\% to $50 \%$ in observer 2. (AUC 0.80-0.67 vs 0.94-0.94)

\section{CONCLUSIONS}

T2W-MRI fused with DWI show a significant increase in diagnostic performance for the assessment of parametrial invasion in early-stage cervical carcinoma.

\section{HIGHLIGHTS}

- Fusion T2W/DWI is superior for assessing parametrial invasion in cervical cancer

- False positive findings of parametrial invasion decreased with fusion T2W/DWI

- Fusion T2W/DWI could improve staging and sequential allocation to the correct treatment strategy.

\section{INTRODUCTION}

Staging of cervical cancer is performed by clinical examination. (1) The 2014 International Federation of Gynecology and Obstetrics (FIGO) stage of cervical cancer is one of the most important factors in determining treatment options. Parametrial invasion is the discriminating factor between FIGO stage IB and IIB disease. FIGO stage IB disease is commonly treated with radical hysterectomy and pelvic lymphadenectomy whereas stage IIB disease is being treated with chemoradiation. ${ }^{(2)}$ When parametrial invasion, lymph node metastases or a combination of other prognostic unfavorable risk factors are recognized on pathologic examination after surgery, adjuvant chemoradiation is advised. ${ }^{(3)}$ Radiation therapy following a radical hysterectomy is associated with higher morbidity and an increase in costs. ${ }^{(3,4)}$ Therefore, adequate preoperative staging is considered essential. ${ }^{(5)}$

Multiple research groups evaluated the predictive value of Magnetic Resonance Imaging (MRI) for parametrial invasion, a systematic review showed MRI to be superior to clinical examination. ${ }^{(6)}$ Recently, the European Society of Gynaecologic Oncology (ESGO) incorporated pelvic T2Weighted (W) MRI as a mandatory workup tool for cervical cancer staging. ${ }^{(7)}$ Nevertheless, evaluation of parametrial invasion on conventional T2W imaging proves to be difficult, as $13-25 \%$ of parametrial invasion is being missed. ${ }^{(6,8)}$

On MRI, exclusion of parametrial invasion is most often defined by a regular hypointense cervical stromal ring, without signs of disruption. (9, 10) Due to tumor growth, pressure, edema and the biological reaction of the surrounding cervical tissue, assessing parametrial invasion on T2W-MRI is prone to false positive results as the hypointense ring is broken by non-tumorous tissue.(11, 12) Diffusion weighted magnetic resonance imaging (DWI) bears the potential to overcome this problem. DWI reflects cell density by measuring water diffusion. Malignant cervical tissue has a higher cell density compared to normal cervical stroma or parametrium. Peri-tumoral edema does not show diffusion restriction which makes it possible to make the differentiation between peri-tumoral edema and tumor. ${ }^{(13)}$

Studies focusing on DWI for the prediction of parametrial invasion are scarce. The potential increase in diagnostic performance of DWI for the evaluation of parametrial invasion has been highlighted in two retrospective single institution trials. ${ }^{(14,15)}$ One study showed some additional benefit of fusion images (T2Wfused with DWI). Two prospective cohort studies could not draw firm conclusions as only a small part of 
their study populations (20 and 25 patients) had DWI sequences available for the interpretation of parametrial invasion. ${ }^{(16,17)}$

This study aims to evaluate the diagnostic performance of diffusion weighted MRI in addition to T2W MRI sequences for assessment of parametrial invasion. In addition,

\section{MATERIALS AND METHODS}

\section{PATIENTS}

From a prospective cohort of patients with histologically proven primary cervical cancer all the patients between April 2011 and June 2016, planned for radical hysterectomy with pelvic lymphadenectomy were selected. (Figure 1, flowchart) The standard diagnostic work-up of patients with early stage disease in our hospital consisted of a gynecological examination under general anesthesia, chest radiography, and MRI of the pelvis. All patients were discussed in a multi-disciplinary team meeting consisting of a nuclear physician, radiologist, pathologist, gynecologic oncologists, medical oncologist, and radiation oncologist. To prevent unnecessary adjuvant treatments and reflect current daily practice, a strict policy for allocation of patients to surgery was advocated; patient with clear or highly suspected parametrial invasion (FIGO IIB) were allocated to chemoradiation. Ethical approval was given and informed consent for the use of (coded) images was waived by the local ethical committee, as the data was analyzed anonymously in accordance with the Institutional Review Board guidelines (IRB no. 16-4-023).

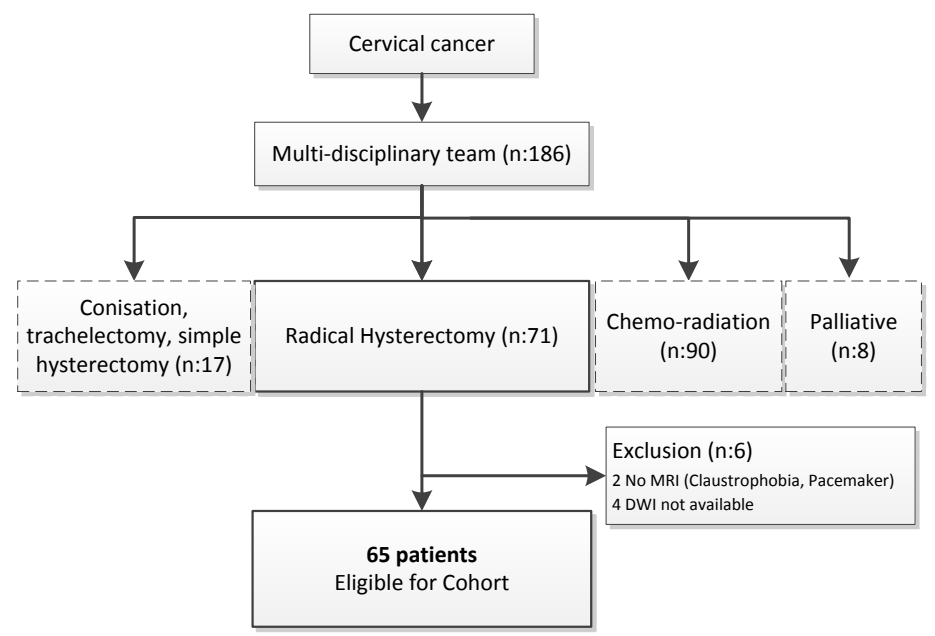

FIGURE 1. Flowchart

\section{MR}

MRI was performed within 8 weeks prior to surgery. All examinations were made on an Intera Achieva; Philips Medical Systems, Best, The Netherlands; or Siemens Magnetom Avanto / Biograph mMR PET-MR, Siemens Healthineers, Erlangen, Germany. 
The majority of the MRI examinations (54\%, n:35) were performed on a 1.5T MRI unit with a phased array surface coil. Patients were placed in a feet-first supine position. The imaging protocol comprised standard 2D T2W fast spin-echo images in three orthogonal directions. The axial and coronal images were angled perpendicular and parallel to the cervical axis, respectively. The remaining MRI examinations (46\%; n:30) were performed using a 3T MRI unit using a similar T2W protocol. For axial DWI a single shot echo planar protocol was performed with b0 as the lowest and b800 $(n=5)$ or b1000 ( $n=60)$ as the highest b-value. ADC maps were automatically generated. Patients received neither bowel preparation nor anti-spasmodic agents during the MRI examinations. The scanning protocol can be found in the Appendix A.

The MR images were retrospectively independently analyzed by two experienced abdominal radiologists (FB and CM with 12 and 8 years of experience in pelvic imaging, respectively) for parametrial invasion of cervical cancer. They were both blinded to the clinical FIGO stage, the pathology of the included patients and the results of the other radiologist or previous reports.

The T2W images alone, T2W combined with the DWI images (T2W/DWI) and the fusion of T2W images with high B-value DWI images (fusion T2W/DWI) were evaluated. For half of the patients first T2W was scored and for the other half T2W/DWI and fusion T2W/DWI was evaluated first. This strategy was used to avoid bias induced by the growing experience of the observer. Second, in order to avoid recall bias, an interval of at least 4 weeks between evaluation of these images was maintained.

The readers were asked to assess the presence of parametrial invasion based on a subjective visual assessment of the images using a semi qualitative 6 -point confidence scale: 0 , definitely no parametrial invasion; 1 , probably no parametrial invasion; 2 , unclear, but likely no parametrial invasion; 3, unclear, but likely parametrial invasion; 4 , probably parametrial invasion; 5 definitely parametrial invasion.

Exclusion of parametrial invasion was considered if the hypointense stromal ring was intact either on T2W or on the fusion images (score 0 or 1). ${ }^{(9)}$ Suspicion of parametrial invasion was raised (score 4 and 5) whenever the stromal ring was disrupted and if tumor signal intensity (nodular or irregular) was seen on the parametrium or bulging in the parametrium. ${ }^{(12)}$ If the stromal ring was disrupted without other signs of parametrial invasion the radiologist scored ( 2 or 3). These same criteria were used for the diffusion and fusion images. The readers used the criteria as a guideline but were free to interpreted the scans based on prior experience. An example is shown in Figure 2.

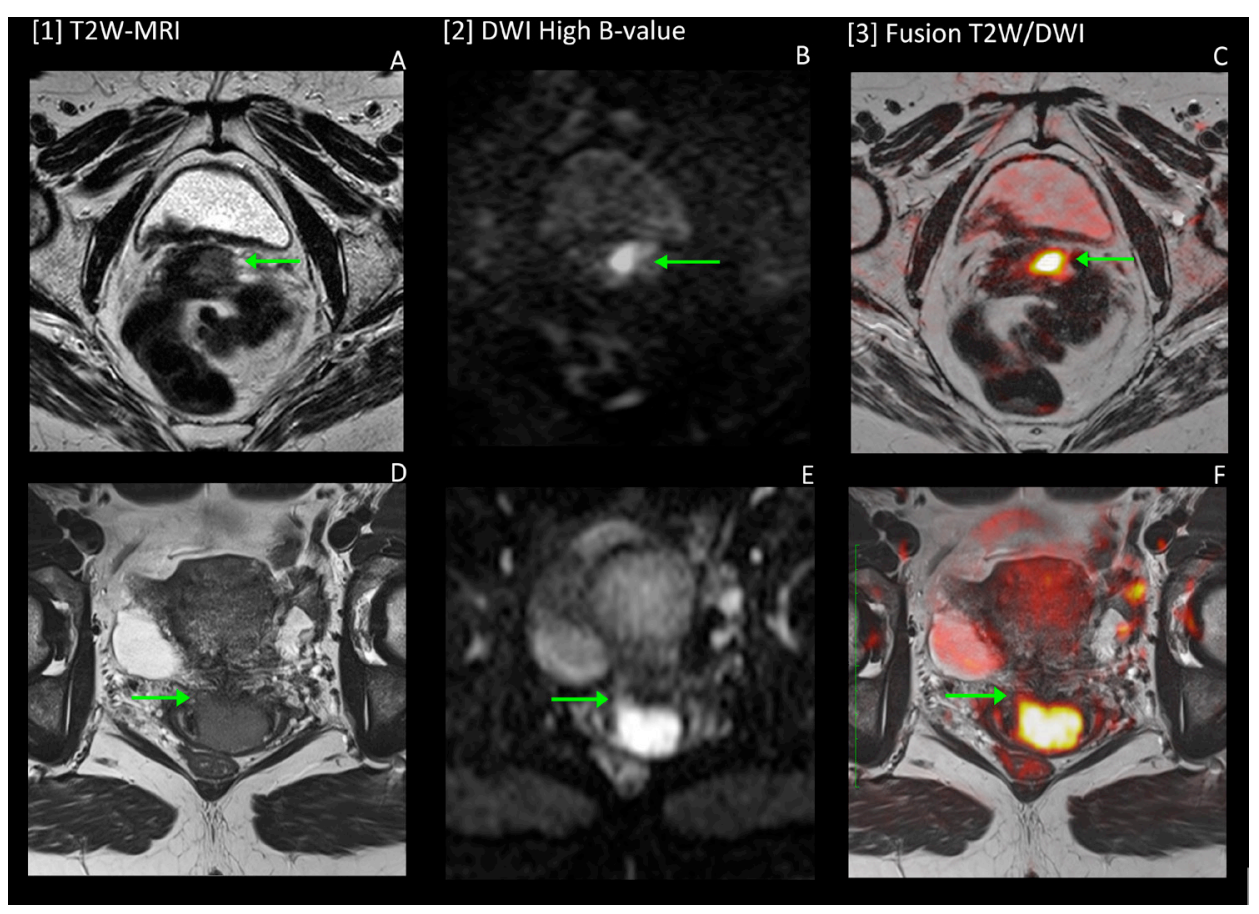

FIGURE 2. T2W MRI, high B-value diffusion and fusion imaging assessing parametrial invasion for cervical carcinoma. Upper pictures (A, B, C): Left side pathology proven parametrial invasion, fusion images (C) increasing certainty. Lower pictures (D, E, F): Right side at pathology no parametrial invasion. T2W image (D) right side stromal ring not clear assessed as possible parametrial involvement. Fusion images (F) downgraded the score to probably no parametrial invasion

For the calculation of performance indices of the diagnostic test, a score of 0-2 was considered as a negative result (no parametrial invasion) and a score of 3-5 was considered as a positive result (parametrial invasion). Additionally, to subjectively evaluate the increase in certainty between T2W/DWI and fusion T2W/DWI, a 5-point Likert scale (observer prevalence rating) was scored.

\section{STANDARD OF REFERENCE}

The reference standard was histological results obtained by an experienced gynecopathologist.

\section{DATA MANAGEMENT AND STATISTICAL ANALYSIS}

Receiver-operating-characteristics $(\mathrm{ROC})$ curves were constructed to determine the diagnostic performance of T2W-MRI, combined T2W/DWI and fusion T2W/DWI for 
Corresponding areas under the ROC curve (AUCs), sensitivity, specificity, and positive and negative predictive values were calculated according to a predefined cut off. Sensitivity and specificity for the different scoring methods were compared using the McNemar test for paired data and predictive values were compared by the method of Leisenring et al. ${ }^{(18)}$ AUCs were compared according to the method described by De Long et al. ${ }^{(19)}$ Interobserver variability was assessed by means of quadratic weighted kappa's: less than 0.20 poor agreement, $0.20-0.40$ fair agreement, $0.40-0.60$ moderate agreement, $0.60-0.80$ good agreement and above 0.81 excellent agreement. ${ }^{(20)}$

Prior to evaluation of the prospective cohort study a sample size calculation was performed. A prevalence of parametrial invasion of $15-25 \%$ (based on literature) was taken into account. ${ }^{(6)}$ Second, an AUC of T2W MRI of $0.75-0.80$ was expected. Approximately 62 patients were needed to show a difference in AUC of $0.10(0.8$ power and alpha 0.05 ). To anticipate $10-15 \%$ exclusions, we needed a prospective cohort of 71 patients.

Statistical analyses were performed using IBM Corp. SPSS Statistics v22.0 (IBM Corp., Armonk, NY), Stata v11.0 (StataCorp LP, Texas) and R (version 3.2.3). All $P$ values were two-sided, and a $P$-value $<0.05$ was considered statistically significant.

\section{POPULATION}

Seventy-one consecutive patients were eligible for this cohort. Two patients were excluded from the cohort as no MRI was performed (claustrophobia or pacemaker) and in four patients no DWI sequences were performed by the referring center. The baseline characteristics of the remaining 65 patients are listed in Table 1.

\begin{tabular}{|c|c|c|}
\hline Age years (range) & & $48(27-82)$ \\
\hline \multirow[t]{5}{*}{ FIGO stage (clinical) } & IA & $3(4 \%)$ \\
\hline & IB1 & $53(82 \%)$ \\
\hline & IB2 & $6(9 \%)$ \\
\hline & $\| \mathrm{A}$ & $2(3 \%)$ \\
\hline & $\| B$ & $1(2 \%)$ \\
\hline \multirow[t]{4}{*}{ Pathology } & scc & $51(78 \%)$ \\
\hline & ADC & $10(15 \%)$ \\
\hline & ASC & $3(5 \%)$ \\
\hline & $\mathrm{ccc}$ & $1(2 \%)$ \\
\hline Time from MRI to operation weeks (range) & & $2.7(0-8)$ \\
\hline Parametrial invasion (histology) & & $8(12.3 \%)$ \\
\hline
\end{tabular}

After surgery, histologic examination identified parametrial invasion in eight patients. In one patient, the procedure was discontinued because of bulky histologicallypositive lymph nodes and obvious parametrial invasion, and the patient was therefore included as positive for parametrial invasion. In one patient FIGO stage IIB disease was suspected, chemo-radiation would have been first choice of treatment but this patient was operated due to excessive tumor blood loss.

\section{DIAGNOSTIC PERFORMANCE FOR ASSESSING PARAMETRIAL INVASION}

Table 2 shows the AUC of the ROC curve for assessment of parametrial invasion for T2W-MRI, T2W/DWI and fusion T2W/DWI evaluation.

Both observers showed a significant increase in AUC from 0.80-0.67 to 0.94-0.94 when the fusion T2W/DWI was used compared to conventional T2W-MRI images $(p=0.03$ and $p<0.01)$. The AUC for combined T2W/DWI compared to either T2W-MRI or fusion T2W/DWI was not significantly different. (Table 2) Intraobserver agreement proved to be good in all evaluation methods (Kappa 0.58-0.65) 
TABLE 2. Interobserver agreement (Kappa) and area under the ROC curve (AUC) for the assessment of parametrial invasion in cervical cancer.

\begin{tabular}{|c|c|c|c|}
\hline & \multirow[t]{2}{*}{ Kappa (SD) } & \multicolumn{2}{|c|}{ AUC (SD) } \\
\hline & & Observer 1 & Observer 2 \\
\hline T2W-MRI & $.57(.12)$ & $.80(.63-.98)$ & $.67(.48-.87)$ \\
\hline Combined T2W/DWI & . 65 (.12) & $.85(.69-1.0)$ & $.85(.67-1.0)$ \\
\hline Fusion T2W/DWI & . 65 (.12) & $.94(.87-1.0)^{*}$ & $.94(.87-1.0)^{*} \mathrm{x} \quad \mathrm{x}$ \\
\hline
\end{tabular}

* $p<0.05$ compared to T2W-MRI. AUC: area under the ROC curve. SD: standard deviation.

Table 3 shows the diagnostic performance indices for T2W-MRI, T2W/DWI and fusion T2W/DWI evaluation. Sensitivity and specificity increased for both observers when using fusion T2W/DWI. For both observers, the positive predictive value increased significantly compared to T2W image evaluation when fusion T2W/DWI images were available (both $p=0.03$ ). No statistically significant differences were found for the other individual parameters. The Likert scale analysis showed that both observers found the additional fusion images useful in $32-34 \%$ of patients.

TABLE 3. Diagnostic performance $(n=65)$ for the assessment of parametrial invasion $(n=8$ on pathologic examination), T2W-MRI versus T2W/DWI and fusion of T2W-MRI with DWI.

\begin{tabular}{lllll}
\hline & \multicolumn{3}{c}{ Diagnostic performance } & \\
& Sensitivity & Specificity & PPV & NPV \\
\hline Observer 1 T2W-MRI & $63(26-89)$ & $79(66-88)$ & $29(11-56)$ & $94(82-98)$ \\
Observer 1 T2W/DWI & $88(47-99)$ & $81(69-90)$ & $37(17-61)$ & $98(88-100)$ \\
Observer 1 Fusion T2W/DWI & $88(47-99)$ & $88(76-95)$ & $50(24-76)^{\star}$ & $98(88-100)$ \\
Observer 2 T2W-MRI & $38(10-74)$ & $82(69-91)$ & $23(6-54)$ & $90(78-96)$ \\
Observer 2 T2W/DWI & $63(26-90)$ & $88(76-95)$ & $42(16-74)$ & $94(83-99)$ \\
Observer 2 Fusion T2W/DWI & $75(36-96)$ & $89(78-96)$ & $50(22-78)^{\star}$ & $96(86-99)$ \\
\hline
\end{tabular}

* p<0.05 compared to T2W MRI PPV: Positive Predictive Value. NPV: Negative Predictive Value

\section{DISCUSSION}

This is the first prospective cohort representing daily clinical practice showing that the fusion of T2W-MRI with DWI results in an increase in diagnostic performance for the assessment of parametrial invasion in clinical early-stage cervical carcinoma. The increase in performance could possibly be explained by more detailed delineation of the cervical cancer with fusion T2W/DWI, making a better discrimination between tumor, pressure induced changes, edema, and/or the biological reaction of the surrounding cervical tissue possible. No significant difference was found between T2W-MRI and combined T2W/DWI without fusion, however a trend in favor of T2W/ DWI was shown for all parameters.

Accurate assessment of parametrial invasion to select the patients that should be allocated to chemoradiation therapy is essential. ${ }^{21,22)}$ If parametrial invasion is recognized after surgery, additional chemoradiation is advised to increase local control and survival, ${ }^{(3)}$ which is associated with more morbidity and higher costs. ${ }^{(4)}$ In the present study, an observed increase in negative and positive predictive values could potentially prevent unnecessary adjuvant treatment after surgery, while increasing the percentage correctly allocated to chemo-radiation.

The present results are in line with two previously published studies on this topic.(14, 15) Park et al. showed in a retrospective study (29\% exclusion due to inadequate MRI) in an Asian population a significant increase of the AUC (.97-.98) particularly in fusion images compared to T2W images. In accordance with our study, the positive predictive value for depicting parametrial invasion increased ${ }^{(14)}$; however, the present study is the first to evaluate this in a prospective cohort.

Interestingly, the difference in the prevalence of parametrial invasion is relatively large; a prevalence of $25 \%$ in the study from Park et al. in comparison to $12 \%$ in the present study. One reason for this difference might be patient selection; $29 \%$ of the patient population in the study of Park et al. ${ }^{(23)}$ had $>\mid B 1$ disease compared to only $15 \%$ in the present cohort, probably due to a more strict allocation of $>\mid \mathrm{B} 1$ disease to chemoradiation by our multi-disciplinary team. As a consequence, the cohort of Park et al. could partly be compromised by a relatively large number of patients with an a priori high suspicion of parametrial invasion, facilitating the diagnostic performance of the test. Compared to the present study, they showed a remarkable higher AUC for conventional T2W-MRI (0.89-0.91 versus $0.80-0.67$ in our population). A recent metaanalysis supports the assumption: a tendency to increased sensitivity was found within 
studies including higher stages cervical cancers. ${ }^{(24)}$ In concordance with the present findings, this systematic review and two recent studies showed a parametrial invasion prevalence of $17 \%$ and $12-13 \%$, respectively. ${ }^{(15,16,24)}$ Compared to the present results, the latter two studies showed a comparable diagnostic performance (AUC 0.76-0.80) for depicting parametrial invasion solely with conventional T2W-MRI.

The only other retrospective study addressing DWI (without fusion) for parametrial invasion found similar results (AUC .91-.95), but their population also consisted for $67 \%$ out of patients with > FIGO IB1 carcinoma's. ${ }^{(15)}$ Besides, in contrast to our study, fusion between the DWI and T2W images was not performed.

Multiple research groups (ConCerv, GOG-278 and SHAPE) currently investigate the feasibility of less radical surgery for low risk cervical cancers. ${ }^{(25-28)} \mathrm{DWI}$ could be useful to identify the 'low risk' patients as the negative predictive value is rather high: $96-98 \%$ in our study. ${ }^{(29,30)}$

The present study has some limitations. First, the study was powered to assess a difference in AUC of 0.10 between conventional and fusion imaging with a prevalence of parametrial invasion of $15-25 \%$. As a consequence, the study was not powered to detect a smaller difference in AUC as seen between combined T2W/DWI and fusion T2W/DWI. In this respect, our study was slightly underpowered, but despite the relatively small number of patients with parametrial invasion a statistical significant increase in AUC was found for fusion T2W/DWI, implying that the positive effect might be even larger than anticipated. Second, as in all cervical cancer studies assessing parametrial invasion, patient selection remains an issue. In retrospect limiting patient selection to FIGO IB1 tumors $(2-4 \mathrm{~cm})$ would have resulted in a more uniform cohort of patients; however, due to the small number of patients this was not an option as the power would not have been sufficient. Another limitation is that higher stages are allocated to chemoradiation therapy, and therefore do not undergo surgery, so the sensitivity of the test would be underestimated. However, as the goal was to address the additional value of DWI this would be the case for both tests. Moreover, a study addressing this issue would be considered non-ethical as this would result in significant triple modality treatment.

Third, due to the nature of the present study, a prospective cohort representing daily practice with introduction of new MRI systems, DWI were performed on both 1.5 and 3T machines. Recently, one meta-analysis showed field strength to be an influencing factor in the assessment of parametrial invasion. ${ }^{(24)}$ As diffusion sequences were subjectively assessed and compared different techniques within the same patient field strength induced bias would not be expected to be of major importance in this study. Moreover, multiple studies showed only small differences across field strengths for abdominal imaging even for quantitative analyses. ${ }^{(31)}$ Due to changes in MRI units, five patients were scanned on a 3 T PET-MRI scanner with a different diffusion protocol with the highest B-value 800 . This protocol was implemented to decrease scanning time. Higher B-values are preferred as the ratio of tumor signal-surroundings ratio is less; however, differences were $<10 \%$ even for quantitative ADC analyses. ${ }^{\left({ }^{32}\right)}$ Therefore, due to the qualitative nature and the relative small difference in B-value, the present results are not expect to be hampered due to the change in protocol. Changes in imaging protocol are common and reflect general practice. In addition, as patients were imaged on units from different vendors, image quality may vary; however, despite the heterogenic population reflecting general daily practice, fusion T2W/DWI imaging increased the diagnostic performance. Therefore, the present study lowers the threshold for implementation in clinical practice.

Treatment of cervical cancer is evolving to favour less radical surgery, neoadjuvant chemotherapy and MRI based brachytherapy. The results of the present study as well as other research groups have shown additional value of MRI in staging of cervical cancer. The present results support the hypothesis that the addition of DWI increases diagnostic performance for the assessment of parametrial invasion even more. When MRI is to be incorporated in a new classification for cervical cancer, diffusion weighted MRI should be considered.

In conclusion, the present results show that conventional T2W-MRI fused with DWI results in an increase in diagnostic performance for the assessment of parametrial invasion in early-stage cervical carcinoma. 


\section{REFERENCE}

1. Jolly S US, Bhatla N, Johnston C, Maturen K. Improving Global Outcomes in Cervical Cancer: The Time Has Come for International Federation of Gynecology and Obstetrics Staging to Formally Incorporate Advanced Imaging. Journal of Global Oncology. 2017:1-6.

2. Salicru SR, de la Torre JF, Gil-Moreno A. The surgical management of early-stage cervical cancer. Curr Opin Obstet Gynecol. 2013;25(4):312-9.

3. Peters WA, 3rd, Liu PY, Barrett RJ, 2nd, Stock RJ, Monk BJ, Berek JS, et al. Concurrent chemotherapy and pelvic radiation therapy compared with pelvic radiation therapy alone as adjuvant therapy after radical surgery in high-risk early-stage cancer of the cervix. J Clin Oncol. 2000;18(8):1606-13.

4. Katanyoo K, Praditsitthikorn N, Tangjitgamol S, Manusirivithaya S, Supawattanabodee B. Cost-utility analysis of treatments for stage IB cervical cancer. J Gynecol Oncol. 2014;25(2):97-104.

5. Pandharipande PV, Choy G, del Carmen MG, Gazelle GS, Russell AH, Lee SI. MRI and PET/CT for triaging stage IB clinically operable cervical cancer to appropriate therapy: decision analysis to assess patient outcomes. AJR Am J Roentgenol. 2009;192(3):802-14

6. Thomeer MG, Gerestein C, Spronk S, van Doorn HC, van der Ham E, Hunink MG. Clinical examination versus magnetic resonance imaging in the pretreatment staging of cervical carcinoma: systematic review and meta-analysis. Eur Radiol. 2013:23(7):2005-18

7. Cibula D, Potter R, Planchamp F, Avall-Lundqvist E, Fischerova D, Haie Meder C, et al. The European Society of Gynaecological Oncology/European Society for Radiotherapy and Oncology/European Society of Pathology guidelines for the management of patients with cervical cancer. Radiother Oncol. 2018;127(3):404-16.

8. Kato T, Takashima A, Kasamatsu T, Nakamura K, Mizusawa J, Nakanishi T, et al. Clinical tumor diameter and prognosis of patients with FIGO stage IB1 cervical cancer (JCOG0806-A). Gynecol Oncol. 2015;137(1):34-

9. Zand KR, Reinhold C, Abe H, Maheshwari S, Mohamed A, Upegui D. Magnetic resonance imaging of the cervix. Cancer Imaging. 2007;7:69-76.

10. Addley H, Moyle P, Freeman S. Diffusion-weighted imaging in gynaecological malignancy. Clin Radiol. 2017;72(11):981-90.

11. Hricak H, Lacey CG, Sandles LG, Chang YC, Winkler ML, Stern JL. Invasive cervical carcinoma: comparison of MR imaging and surgical findings. Radiology. 1988;166(3):623-31.

12. Shweel MA, Abdel-Gawad EA, Abdel-Gawad EA, Abdelghany HS, Abdel-Rahman AM, Ibrahim EM. Uterine cervical malignancy: diagnostic accuracy of MRI with histopathologic correlation. J Clin Imaging Sci. 2012;2:42.

13. Charles-Edwards EM, Messiou C, Morgan VA, De Silva SS, McWhinney NA, Katesmark M, et al. Diffusionweighted imaging in cervical cancer with an endovaginal technique: potential value for improving tumor detection in stage la and lb1 disease. Radiology. 2008;249(2):541-50.

14. Park JJ, Kim CK, Park SY, Park BK. Parametrial invasion in cervical cancer: fused T2-weighted imaging and high-b-value diffusion-weighted imaging with background body signal suppression at $3 \mathrm{~T}$. Radiology. 2015;274(3):734-41.

15. Qu JR, Qin L, Li X, Luo JP, Li J, Zhang HK, et al. Predicting Parametrial Invasion in Cervical Carcinoma (Stages IB1, IB2, and IIA): Diagnostic Accuracy of T2-Weighted Imaging Combined With DWI at 3 T. AJR Am J Roentgenol. 2018;210(3):677-84

16. Bourgioti C, Chatoupis K, Rodolakis A, Antoniou A, Tzavara C, Koutoulidis V, et al. Incremental prognostic value of MRI in the staging of early cervical cancer: a prospective study and review of the literature. Clin Imaging. 2016:40(1):72-8.

17. Downey K, Attygalle AD, Morgan VA, Giles SL, MacDonald A, Davis M, et al. Comparison of optimised endovaginal vs external array coil T2-weighted and diffusion-weighted imaging techniques for detecting suspected early stage (IA/B1) uterine cervical cancer. Eur Radiol. 2016;26(4):941-50.

18. Leisenring W, Alonzo T, Pepe MS. Comparisons of predictive values of binary medical diagnostic tests for paired designs. Biometrics. 2000;56(2):345-51.

19. DeLong ER, DeLong DM, Clarke-Pearson DL. Comparing the areas under two or more correlated receiver operating characteristic curves: a nonparametric approach. Biometrics. 1988;44(3):837-45

20. Viera AJ, Garrett JM. Understanding interobserver agreement: the kappa statistic. Fam Med. 2005:37(5):360-3.

21. Green J, Kirwan J, Tierney J, Vale C, Symonds P, Fresco L, et al. Concomitant chemotherapy and radiation therapy for cancer of the uterine cervix. Cochrane Database Syst Rev. 2005(3):CD002225.

22. Sturdza A, Potter R, Fokdal LU, Haie-Meder C, Tan LT, Mazeron R, et al. Image guided brachytherapy in locally advanced cervical cancer: Improved pelvic control and survival in RetroEMBRACE, a multicenter cohort study. Radiother Oncol. 2016;120(3):428-33.

23. Landoni F, Maneo A, Colombo A, Placa F, Milani R, Perego P, et al. Randomised study of radical surgery versus radiotherapy for stage Ib-lla cervical cancer. Lancet. 1997;350(9077):535-40

24. Woo S, Suh CH, Kim SY, Cho JY, Kim SH. Magnetic resonance imaging for detection of parametrial invasion in cervical cancer: An updated systematic review and meta-analysis of the literature between 2012 and 2016. Eur Radiol. 2017.

25. Lee JY, Youm J, Kim JW, Cho JY, Kim MA, Kim TH, et al. Identifying a low-risk group for parametrial involvement in microscopic Stage IB1 cervical cancer using criteria from ongoing studies and a new MRI criterion. BMC Cancer. 2015;15:167.

26. Crosbie EJ, Einstein MH, Franceschi S, Kitchener HC. Human papillomavirus and cervical cancer. Lancet. 2013;382(9895):889-99.

27. Cohen PA, Jhingran A, Oaknin A, Denny L. Cervical cancer. Lancet. 2019:393(10167):169-82.

28. Group FIS. Quadrivalent vaccine against human papillomavirus to prevent high-grade cervical lesions. N Engl J Med. 2007:356(19):1915-27.

29. Li X, Wang L, Li Y, Song P. The Value of Diffusion-Weighted Imaging in Combination With Conventional Magnetic Resonance Imaging for Improving Tumor Detection for Early Cervical Carcinoma Treated With Fertility-Sparing Surgery. Int J Gynecol Cancer. 2017;27(8):1761-8. 
30. He F, Du J, Chen X, He L. Assessment of Parametrial Involvement in Early Stages Cervical Cancer With Preoperative Magnetic Resonance Imaging. Int J Gynecol Cancer. 2018

31. Rosenkrantz AB, Oei M, Babb JS, Niver BE, Taouli B. Diffusion-weighted imaging of the abdomen at 3.0 Tesla: image quality and apparent diffusion coefficient reproducibility compared with 1.5 Tesla. J Magn Reson Imaging. 2011;33(1):128-35.

32. Gladwish AP, Han K, Foltz WD. Variation in apparent diffusion coefficient measurements among women with locally advanced cervical cancer. Radiother Oncol. 2015;117(3):532-5.

\section{APPENDIX A}

SUPPLEMENTARY TABLE 1. Magnetic Resonance Imaging Protocol

\begin{tabular}{lllllllll}
\hline & TR & TE & ETL & NSA & $\begin{array}{l}\text { Acquisition } \\
\text { size }(\mathrm{mm})\end{array}$ & Slices & $\begin{array}{l}\text { Acquisition } \\
\text { time }(\mathrm{min})\end{array}$ & $\begin{array}{l}\text { Epi factor/ } \\
\text { B-value }\end{array}$ \\
\hline T2W 1.5 T & $3200-3427$ & $100-150$ & $18-24$ & $3-4$ & $0.98 \times 0.98 \times 3-5$ & $24-48$ & $3.15-5.33$ & - \\
T2W 3.0 T* & 7000 & 150 & 28 & 2 & $0.98 \times 0.98 \times 3-4$ & 40 & 3.15 & - \\
DWI 1.5 T & $3200-4127$ & $65-80$ & - & $4-6$ & $1.96 \times 2.5 \times 4-6$ & $32-36$ & $3-4$ & $59, \mathrm{b0}-\mathrm{b} 1000$ \\
DWI 3.0 T* & 2000 & 57 & - & $4-6$ & $2.5 \times 2.5 \times 3$ & $36-42$ & 3.20 & $75, \mathrm{b0}-\mathrm{b} 1000$ \\
\hline
\end{tabular}

* Except for five patients scanned on a 3 T PET-MR with comparable T2W and a DWI protocol with a highest b-value of

800 due to the introduction of a new scanner and subsequent scan protocol. SPAIR fat suppression techniques were used in all patients. TR: repetition time; TE: echo time; ETL: echo train length; NSA: number of signal averages; Epi: echo planar imaging 


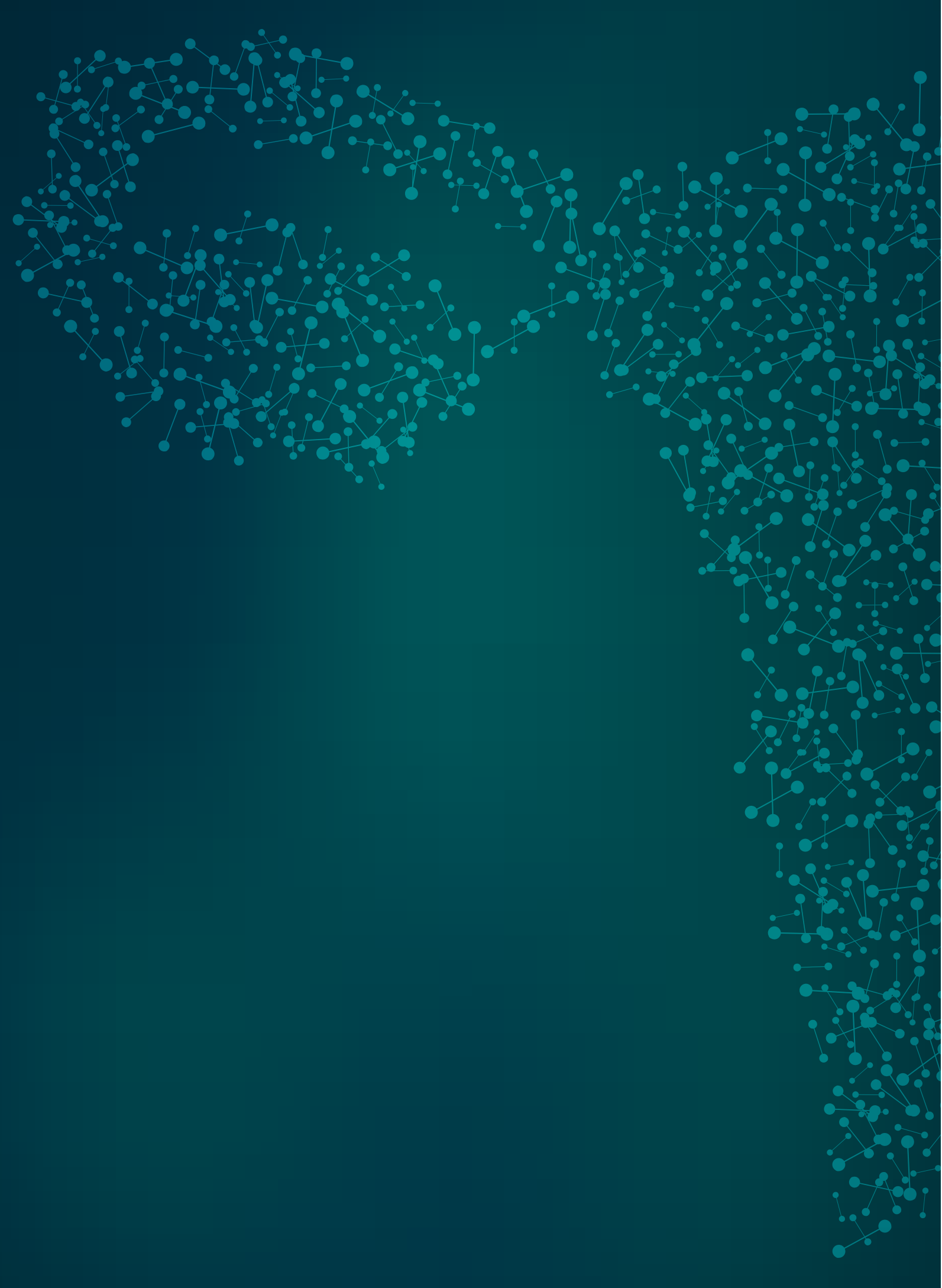

EVALUATION OF VARIOUS APPARENT DIFFUSION COEFFICIENT MEASUREMENT TECHNIQUES IN PRE-OPERATIVE STAGING OF EARLY CERVICAL CARCINOMA.

Mongula JE

Bakers FCH

Slangen BFM

van Kuijk SMJ

Kruitwagen RFPM

Mihl C 


\section{ABSTRACT}

\section{PURPOSE}

Diffusion weighted MRI parameters such as apparent diffusion coefficient (ADC) hold the potential for more reliable staging of cervical cancer. We compared 2D region of interest (ROI) measurement techniques to 3D tumor analysis in the evaluation of different $A D C$ parameters for cervical cancer. Secondly, we aimed to evaluate the utility of ADC for assessing parametrial involvement and/or lymph node metastases.

\section{METHODS}

This prospective patient cohort registered histologically proven cervical cancer patients who underwent pre-operative MRI with T1, T2-weighted, and axial DWI sequences. Retrospectively, two observers independently and blindly scored mean, minimum, and maximum ADC of the cervical tumor using three methods: a) 3D-Tumor analysis b) single freehand ROI at the largest diameter (2D-Slice), and c) single circular ROI (2D-Circle). A third observer (blinded) scored parametrial and lymph node involvement on T1 and T2W sequences. Parametrial and/or pelvic lymph node involvement were determined by surgical-pathologic results. The diagnostic performance of ADC for predicting the latter was evaluated by ROC curve, uni and multivariate analyses.

\section{RESULTS}

Of 58 included patients, parametrial and/or pelvic lymph node involvement was found in 9 and 11 patients, respectively. Mean $A D C\left(A D C_{\text {Mean }}\right)$ was least dependent on the ROI technique, with interobserver variability (ICC: 0.88-0.90) and linear correlation (Pearson's $r$ : 0.95-0.96) across ROI techniques. To the contrary, minimal and maximal $A D C$ were significantly influenced by $2 \mathrm{D}$ single ROI techniques. $A D C_{\text {Men }}$ was shown to be an independent predictor of parametrial (AUC: $0.80-0.86$; OR: 16, 1.4-178) and/or lymph node involvement (AUC: 0.74-0.79; OR: 5.1, 1.1-24).

\section{CONCLUSION}

Single ROI measurements are a reliable method for determining $\mathrm{ADC}_{\text {Mean }}$ in cervical cancer. Second, $\mathrm{ADC}_{\text {Mean }}$ serves as a potential parameter for prediction of parametrial involvement prior to radical hysterectomy.

\section{HIGHLIGHTS}

- $A D C$ measurements are influenced by the used measurement technique

- Mean ADC values can reliable be measured with single ROI measurements

- Minimal and maximal ADC values should be interpreted with caution

- Mean ADC is a promising biomarker for assessing parametrial involvement

50 | PART ॥ CHAPTER 3

\section{INTRODUCTION}

Cervical carcinoma of stage IB1-IIA on the 2014 International Federation of Gynaecology and Obstetrics (FIGO) staging system is generally treated by radical hysterectomy combined with pelvic lymphadenectomy. ${ }^{(1)}$ However, approximately $20 \%$ of parametrial involvement (FIGO stage $\geq \mid \mathrm{IB}$ ) or lymph node metastases are missed at the clinical staging. ${ }^{(2,3)}$ As a consequence, these patients may need additional chemoradiation treatment after surgery, resulting in further complications and higher costs. ${ }^{(4)}$ Recently, the European Society of Gynaecologic Oncology (ESGO) incorporated pelvic T2W-MRI as a mandatory workup tool for cervical cancer staging:(5) however, the role of DWI sequences for pre-operative staging is not clear. ${ }^{(6,7)}$ Studies assessing T2W-MRI parameters for parametrial involvement, found a high negative but a rather low positive predictive value. ${ }^{(7-10)}$ DWI studies on cervical cancer have focused on evaluating the ADC parameter that quantifies diffusion restriction. ${ }^{(11)}$ Low mean ADC $\left(A D C_{\text {Mean }}\right)$ values are associated with an increased incidence of parametrial invasion. (12, 13) Although assessments of other risk factors for disease recurrence such as lymph node metastases may be conflicting, ${ }^{(13,14)} A D C_{\text {Mean }}$ values were associated with survival and risk of recurrence in three retrospective studies. ${ }^{(13,15,16)}$

The optimization of ADC measurements is of great importance. ${ }^{(12,13)}$ In most studies, ADC is measured from the slice with the largest tumor diameter, using either a single circlelike Region of Interest (ROI), ${ }^{(12,17)}$ multiple small circle-like ROIs, ${ }^{(13)}$ or a single freehand $\mathrm{ROI}$ containing all visible tumor on that slice. ${ }^{(16)}$ In addition to two-dimensional (2D) methods, a three-dimensional (3D) method has been used, in which a freehand ROI containing all visible tumor is drawn on all subsequent slices. It has been hypothesized that $3 \mathrm{D}$ analysis is superior to $2 \mathrm{D}$ methods, with a reduction in variability due to the absence of having to select the appropriate 2D-ROI, especially in heterogenic tumors. ${ }^{118,19)}$ However, research in other tumor types has revealed conflicting results regarding observer variability with usage of different $\mathrm{ROI}$ techniques. ${ }^{(20-23)}$ To date, it remains unclear whether 3D-ROI are superior to 2D-ROI in the evaluation of cervical cancer, and as 3D-ROIs are more time consuming, a single representative 2D-ROI may be preferable in clinical practice. ${ }^{(19)}$

Besides the variation in ROI techniques, DWI evaluation was performed by a single observer in most cervical cancer studies, ${ }^{(12,13,16-19)}$ and a variety of ADC parameters $\left(A D C_{\text {Mean' }}\right.$ minimum $A D C_{\text {Min' }}$ and maximum $A D C_{\text {Max }}$ ) have been assessed. The clinical value of $A D C_{\text {Min' }} A D C_{\text {Max }}$ and whether these $A D C$ parameters are reproducible and sufficiently stable to support their use in clinical practice remains to be determined. ${ }^{(13)}$ 
The primary aim of this study was to compare three ADC measurement techniques: a) 3D whole tumor analysis (3D-Tumor), b) a 2D single $\mathrm{ROI}$ at the largest diameter (2D-Slice), and c) a 2D single circular ROI at the largest diameter (2D-Circle). Secondly, we aimed to evaluate the predictive value of the ADC parameters for parametrial and/ or lymph node involvement.

\section{METHODS AND MATERIALS}

\section{PATIENTS}

This cohort study performed between April 2011 and July 2016 prospectively registered patients diagnosed with histologically proven primary cervical cancer and eligible for radical hysterectomy with pelvic lymphadenectomy. The diagnostic workup of patients consisted of a gynecological examination, chest X-ray, and MRI of the pelvis. As our center expected to have a new 3T MRI and 3T PET-MRI scanner installed in the near future, the study design accounted for changes in scan protocols by allowing state of the art MRI with updated protocols. All patients were discussed by a multi-disciplinary team consisting of a nuclear physician, radiologist, pathologist, gynecologic oncologists, medical oncologist, and radiation oncologist. Patients with a history of previous treatment (e.g., radiation and/or chemotherapy) and/or failure to visualize the cervical cancer on T2 and/or DWI were excluded from this study. Ethical approval for the study was given and the requirement for informed consent for the use of (coded) images was waived by the local ethical committee as the data were retrospectively analyzed anonymously in accordance with the Institutional Review Board guidelines (IRB:16-4-023)

\section{MR}

MRI was performed within 8 weeks prior to surgery. The majority of the MRI examinations $(n=33,57 \%)$ were performed on a 1.5 T MRI unit with a phased array surface coil. Patients were placed in a feet-first supine position. The imaging protocol consisted of standard 2D T2W fast spin echo images in three orthogonal directions. The axial and coronal images were angled perpendicular and parallel to the cervical axis, respectively. The remaining MR examinations $(n=25,43 \%)$ were performed on a $3 T$ MRI unit using a similar T2W protocol. A single-shot echo planar protocol was used for the axial DWI, with b-values of 0 and $1000 \mathrm{~s} / \mathrm{mm}^{2}$ (b-values of 0 and $800 \mathrm{~s} / \mathrm{mm}^{2}$ were used in four patients), and ADC maps were automatically generated. In all patients, additional T1W sequences were acquired to facilitate the assessment of pelvic lymph nodes. Patients received neither bowel preparation nor anti-spasmodic agents during the MRI examinations. (Appendix, scan protocol.) All examinations were made on an Intera Achieve; Philips Medical Systems, Best, The Netherlands; or Siemens Magnetom Avanto / Biograph mMR PET-MR, Siemens Healthineers, Erlangen, Germany.

The MR images were retrospectively independently analyzed by two experienced observers (CM 8 and JEM 4 years of experience) who were blinded to the patient information and outcomes. Both observers measured $A D C_{\text {Mean' }} A D C_{\text {Min }}$ and $A D C_{\text {Max }}$ 
values within ROls created using the following three methods (Figure 1):

a) (3D-Tumor) by placing freehand ROls along the border of the suspected tumor (diffusion restricted area) on all slices of the axial ADC maps;

b) (2D-Slice) by placing one freehand $\mathrm{ROI}$ incorporating all visible tumor at the suspected largest tumor diameter on the axial ADC maps:

c) (2D-Circle) by placing a circular $\mathrm{ROI}$ with a surface area of $1 \mathrm{~cm}^{2}$ at the suspected largest tumor diameter, or in the case of a smaller tumor, an ROI as large as possible.

No cystic or necrotic tumor areas were included in the ROIs. All measurements were scored with morphological confirmation of the location of the tumor on axial, coronal, and sagittal T2W images, as well as DWI sequences. The maximum diameter of the cervical tumor were measured on the axial and coronal T2W images. The 3D-Tumor $A D C$ measurements were assessed in consensus by both reviewers as they were used as a reference standard. A third observer (FB, 12 years of experience) scored the likelihood of parametrial involvement on T2W images and lymph node metastases on T2W and T1W images. The imaging criteria for the assessment of parametrial involvement are shown in the Appendix. This observer was blinded to the DWI, ADC, and clinical outcomes. Measurements were performed on a dedicated Osirix v5.9 DICOM system (Pixmeo SARL, Bernex Geneva, Switzerland).

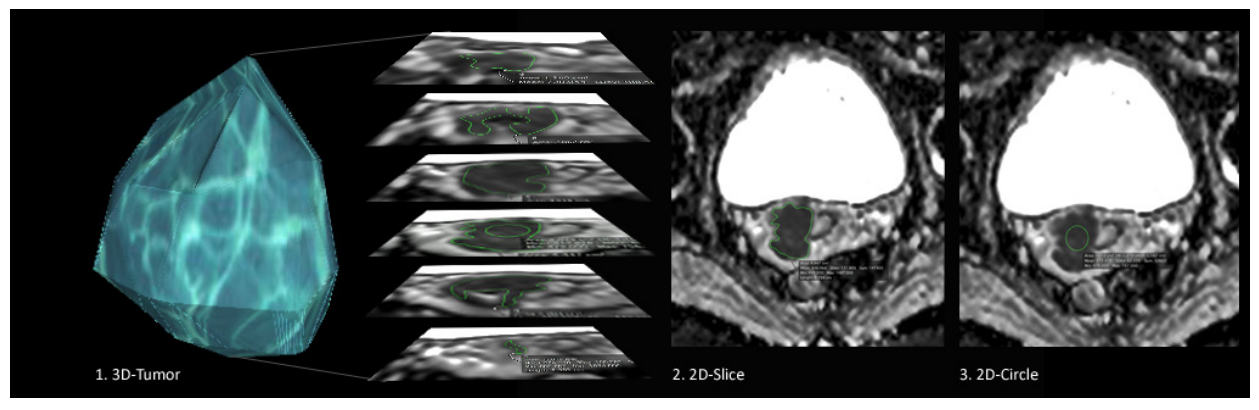

FIGURE 1. ADC measurement techniques for cervical cancer

\section{REFERENCE STANDARD, DATA MANAGEMENT AND STATISTICAL ANALYSIS}

The reference standard was histological results obtained by the pathologist. Baseline characteristics are described as mean $\pm S D$, or median (IQR) for non-normally distributed parameters. Proportions (\%) are used for categorical values. The mean values of the ADC measurement methods were compared with a paired sample $t$-test, and correlations were assessed with Pearson's correlation coefficient.

For all analyses, the results of observer 1 (CM; experienced abdominal radiologist) were used. Interobserver variability was quantified with the intraclass correlation coefficient (ICC). ROC curves were constructed to determine the corresponding AUCs for $A D C_{\text {Mean }}$ values obtained from the ADC measurement methods (3D-Tumor, 2D-Slice, and 2D-Circle), and for determination of parametrial and/or lymph node involvement. The AUCs were compared according to the method described by De Long et al. ${ }^{(24)}$ The optimal ADC cut-off value was determined according to the point nearest to the upper left corner in the ROC curve. Univariable and multivariable logistic regression analyses were performed to calculate odds ratios (OR) for the use of ADC for predicting parametrial and lymph node involvement. Regression analysis was performed to determine the association between ADC measurements, tumor diameter on MRI, and subjective MRI analysis of parametrial involvement and lymph node metastases.

Statistical analyses were performed using SPSS Statistics v20.0 (IBM SPSS Inc., Chicago, III) and Stata v11.0 (StataCorp LP, Texas). P-values less than 0.05 were considered statistically significant. 


\section{RESULTS}

\section{PATIENTS}

Seventy-two consecutive patients were eligible for this study. Fourteen patients were excluded from the cohort because of claustrophobia or pacemaker ( $n=2, n \circ M R I)$, insufficient MRI image quality ( $n=4$, artefacts, no reliable ADC map), lack of DWI sequences ( $n=4$, MRI performed in referral centre), or failure to visualize cancer on the MRI $(n=4)$. A final total of 58 patients were included in this cohort study (Figure 2, Flowchart). Baseline characteristics of the patients are listed in Table 1.

TABLE 1. Baseline characteristics (n: 58 )

\begin{tabular}{ll}
\hline Age years (range) & $47(27-82)$ \\
FIGO stage (clinical) & IA2 $1(2 \%)$ \\
& IB1 $49(84 \%)$ \\
& IB2 $5(9 \%)$ \\
Pathology & IIA $2(3 \%)$ \\
Tumor subtype & $1(2 \%)$ \\
& SCC $45(78 \%)$ \\
& ADC $9(15 \%)$ \\
Parametrial involvement & ASC $3(5 \%)$ \\
Lymph node metastases & CCC $1(2 \%)$ \\
Time from scan to operation weeks (range) & $9(15 \%)$ \\
\hline All variables are described as mean \pm SD or median (IQR) for non-normally distributed parameters. Proportions (\%) were & \\
\hline
\end{tabular}

All variables are described as mean \pm SD or median (IQR) for non-normally distributed parameters. Proportions (\%) were used for categorical values. SCC: squamous cell carcinoma; ADC: adenocarcinoma; ASC: adenosquamous carcinoma; CCC: clear-cell carcinoma

After surgery, histologic examination identified parametrial involvement in 9 patients (7 patients with parametrial invasion and 2 with pathologic parametrial lymph nodes) and pelvic lymph node metastases in 11 patients. In one patient, the surgical procedure was discontinued because of bulky histologically-positive lymph nodes and obvious parametrial involvement, and the patient was therefore included as positive for parametrial involvement.

\section{ADC MEASUREMENT TECHNIQUES}

The $A D C_{\text {Mean }}$ values differed significantly between all three $R O I$ methods $(p<0.01)$. Despite absolute differences, the Pearson's correlation coefficients showed near perfect correlations between all three ROI methods for the $\mathrm{ADC}_{\text {Mean }}$ values (Table 2). The regression analysis indicates a linear relationship for $A D C_{\text {Mean' }}$ with a regression coefficient of 0.92 between 3D-Tumor and 2D-Circle measurements (Figure 3).
The 2D-Tumor techniques for $\mathrm{ADC}_{\text {Min }}$ and $A D C_{\text {max }}$ showed only moderate and poor correlations, respectively, with 3D-Tumor (Table 2). Interobserver variability (Table 2) was excellent for $\mathrm{ADC}_{\text {Mean }}$ and average to good for $\mathrm{ADC}_{\text {Min }}$ and $A D C_{\text {Max }}$ (ICCs $=0.88-0.90$, $0.67-0.70$, and $0.63-0.75$, respectively). Additional analyses showed no significant differences in $A D C_{\text {Mean }}$ between the 1.5 and $3 T$ groups $(p=0.18)$.

\section{ADC AS A PREDICTOR OF PARAMETRIAL INVOLVEMENT OR LYMPH NODE METASTASIS IN CERVICAL CANCER}

The $A D C_{\text {man }}$ parameter showed the highest potential (highest AUCs), which was combined with the lowest interobserver variability (Table 2), and this parameter was therefore evaluated in the univariable and multivariable analyses. For ADC comparable results were found for all ROI techniques. The AUCs of $A D C_{\text {Mean' }} A D C_{\text {Min, }}$ and $A D C_{\operatorname{Max}}$ for predicting parametrial involvement, lymph node metastasis, and the combination of parametrial or lymph node involvement are shown in Table 3.

The univariable analyses showed subjective assessment of the parametrium on T2WMRI to be a significant risk factor for parametrial involvement (Table 4). The univariable and multivariable analyses showed 3D-Tumor $\mathrm{ADC}_{\text {mean }}$ to be an independent predictor of parametrial involvement compared to tumor diameter and subjective assessment of the parametrium on T2W-MRI (OR:16, 1.4-178; Table 4). For either parametrial or lymph node metastasis the ORs of an $\mathrm{ADC}_{\text {Mean }}$ value $<0.94$ was also statistically significant (OR 5.1, 1.1-24; $p<0.05$; Table 5).

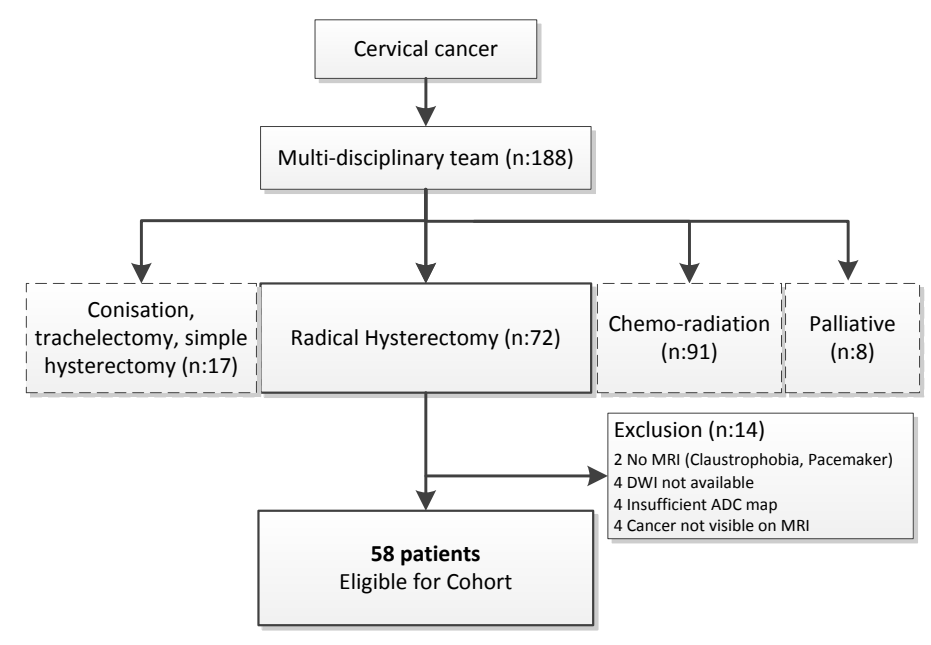

FIGURE 2. Flowchart 
TABLE 2. $A D C_{\text {Mean, }} A D C_{\text {Min, }}$ and $A D C_{\text {Max }}$ values for all three measurement techniques. Interobserver variability (ICC) and Pearson's correlation coefficient (r) between 3D-Tumor and 2D ROI techniques 3D-Tumor 2D-Slice 2D-Circle 2D-Slice $2 \mathrm{D}$-Circle 2D-slice 2D-Circle mean (SD) mean (SD) mean (SD)

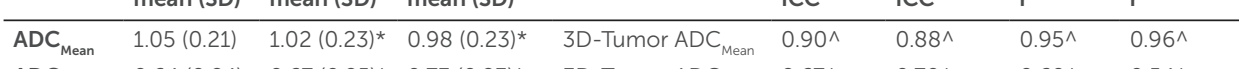

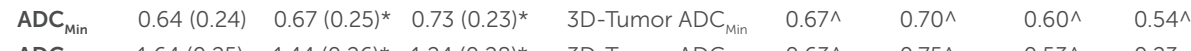

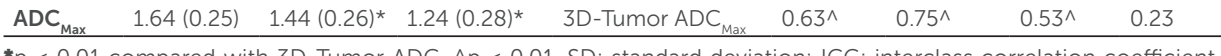

* $p<0.01$ compared with 3D-Tumor ADC. $\wedge p<0.01$. SD: standard deviation; ICC: interclass correlation coefficient, $r$ :

Pearson's correlation coefficient.

TABLE 3. AUC values for $A D C_{\text {Mean' }} A D C_{\text {Max' }}$ and $A D C_{\text {Min }}$ for predicting parametrial, lymph node, and the combination of parametrial and lymph node metastasis

\begin{tabular}{|c|c|c|c|c|c|c|}
\hline ROI Technique & $A D C$ & $\begin{array}{l}\text { Cut-off } \\
\times 10^{-3} \\
\mathrm{~mm} / \mathrm{s}\end{array}$ & $\begin{array}{l}\text { AUC } \\
\text { (SD) }\end{array}$ & $\begin{array}{l}\text { Parametrial } \\
\text { Involvement (n=9) }\end{array}$ & $\begin{array}{l}\text { Lymph Node } \\
\text { Involvement (n=11) }\end{array}$ & $\begin{array}{l}\text { Parametrial or Lymph } \\
\text { Node Involvement } \\
(n=14)\end{array}$ \\
\hline \multirow{3}{*}{ 3D-Tumor } & $\mathrm{ADC}_{\text {Mean }}$ & 0.94 & & $0.81(0.70-0.92)$ * & $0.67(0.51-0.82)$ & $0.74(0.60-0.88) *$ \\
\hline & $A D C_{\max }$ & 1.55 & & $0.52(0.30-0.74)$ & $0.63(0.47-0.80)$ & $0.59(0.41-0.77)$ \\
\hline & $A D C_{\operatorname{Min}}$ & 0.57 & & $0.82(0.71-0.94)$ * & $0.60(0.40-0.80)$ & $0.72(0.57-0.87) * *$ \\
\hline 2D-Slice & $A D C_{\text {Mean }}$ & 0.89 & & $0.80(0.79-0.91)$ * & $0.68(0.52-0.83)$ & $0.74(0.60-0.87)$ * \\
\hline 2D-Circle & $A D C_{\text {Mean }}$ & 0.88 & & $0.86(0.76-0.95)$ * & $0.72(0.57-0.87) * *$ & $0.79(0.67-0.92)$ * \\
\hline
\end{tabular}

${ }^{*} \mathrm{p}<0.01 * *_{\mathrm{p}}<0.05$

TABLE 4. Uni- and multivariate analysis of $3 \mathrm{D}-$ Tumor $\mathrm{ADC}_{\text {Mean }}$ as an independent parameter for predicting parametrial involvement

\begin{tabular}{|c|c|c|c|}
\hline & $A \cup C$ (SD) & Odds ratio (univariate) & Odds ratio (multivariate)^ \\
\hline $\mathrm{ADC}_{\text {Mean }}<0.94 \times 10^{-3} \mathrm{~mm} / \mathrm{s}$ & $0.81(0.70-0.92)^{*}$ & $18.1(2.1-158)^{\star}$ & $16.0(1.4-178)^{\star \star \star}$ \\
\hline FIGO stage $<1 \mathrm{~B}$ & $0.47(0.26-0.68)$ & $0.35(0.03-4.3)$ & \\
\hline PA type (SCC vs. other) & $0.57(0.36-0.79)$ & $2.0(0.4-9.1)$ & - \\
\hline DM tumor T2W-MRI & $0.68(0.50-0.85)$ & $1.4(0.8-2.4)$ & $0.8(0.3-1.8)$ \\
\hline Suspicion of PMI on T2W-MRI & $0.77(0.60-0.93)$ & $1.8(1.1-2.9)^{*}$ & $1.6(0.9-2.7)$ \\
\hline
\end{tabular}

SCC: squamous cell carcinoma, PA: pathology, DM: diameter, PMI: parametrial invasion
TABLE 5. Uni- and multivariate analysis of 3D-Tumor $A D C_{\text {mean }}$ as an independent parameter for parametrial or lymph node involvement

\begin{tabular}{|c|c|c|c|}
\hline & AUC (SD) & Odds ratio (univariate) & Odds ratio (multivariate)^ \\
\hline$A D C_{\text {Meantotal }}<0.94 \times 10^{-3} \mathrm{~mm} / \mathrm{s}$ & $0.74(0.60-0.88)^{\star}$ & $6.0(1.6-22)^{\star}$ & $5.1(1.1-24)^{\star *}$ \\
\hline FIGO stage $<1 \mathrm{~B}$ & $0.49(0.31-0.66)$ & $0.6(0.1-7.4)$ & \\
\hline PA type (PCC vs. other) & $0.59(0.41-0.77)$ & $2.5(0.7-9.5)$ & \\
\hline DM tumor MRI & $0.62(0.47-0.77)$ & $1.3(0.8-2.1)$ & $1.0(0.5-1.9)$ \\
\hline Suspicion of PMI T2W-MRI & $0.74(0.59-0.88)^{\star}$ & $2.4(1.3-4.3)^{*}$ & $1.5(0.9-2.4)$ \\
\hline Suspicion of lymph node MRI & $0.47(0.30-0.64)$ & $0.9(0.5-15)$ & $0.7(0.4-13)$ \\
\hline
\end{tabular}

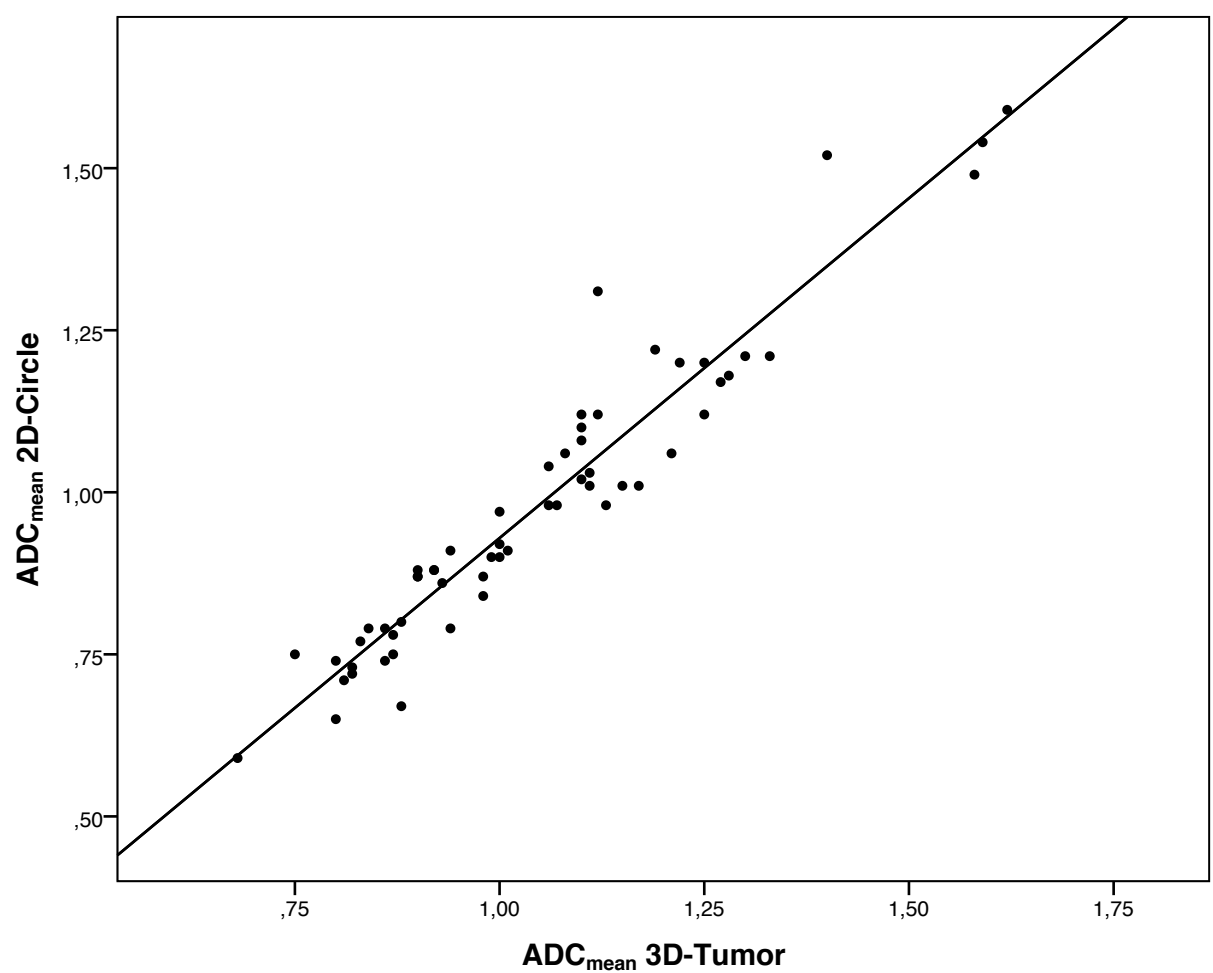

FIGURE 3. Scatterplot of $A D C_{\text {mean }}$ values from 3D-Tumor and 2D-Circle ROIs, including regression analysis 


\section{DISCUSSION}

Our results support the use of a 2D-ROI drawn at the slice of the largest tumor diameter for the measurement of $A D C_{\text {Mean }}$. Moreover, our results suggest $A D C_{\text {Mean }}$ as an independent predictor for parametrial involvement and/or lymph node metastases in early stage cervical carcinoma.

Of the three $A D C$ parameters evaluated, the $A D C_{\text {Mean }}$ value showed the lowest interobserver variability. As the linear associations between $3 \mathrm{D}$ and $2 \mathrm{D}$ techniques were high; the $2 \mathrm{D}$ measurements were able to represent whole tumor $A D C_{\text {Mean }}$. To allow for comparisons between studies using different ROI techniques, correction factors to adjust for the linear correlation should be used, which should help justify the use of the less time-consuming options.

For $A D C_{\text {Min }}$ and $A D C_{\text {Max' }}$, the differences between the ROI techniques were significant, without a strong linear agreement. Therefore, these parameters should be interpreted with caution. The discrepancies between the techniques could be explained by single ADC hotspots within the tumor, which were shown by 3D-tumor analysis but missed in the single ROI methods. An alternative method to potentially overcome this problem is histogram analysis to further characterize the diffusion properties of cervical cancer. Moreover, with the exclusion of outlying values, more reliable and reproducible measurements should be obtainable. ${ }^{(18)}$ While histogram analysis is an evolving method for analyzing DWI, 3D delineation of the whole tumor, as performed in a limited number of studies, remains time consuming. ${ }^{(25-27)}$

In our study, 3D-tumor analyses were performed by two readers in consensus because interobserver variability was suggested to be the biggest source of error concerning ADC measurements in other cancer types. Gladwish et al. evaluated ADC prior to radiation treatment and found a difference of $10 \%$ between 2D and 3D techniques, with good interobserver variability. ${ }^{(28)}$ The size of a circle-like $\mathrm{ROI}$ is important in this respect, ${ }^{(29)}$ with breast and rectal carcinoma studies suggesting that a small ROI contributes to greater variability in ADC in comparison with a freehand ROI delineating as much as possible of the visible tumor. ${ }^{(19,22,30}$

To date, the correlation between lymph node metastases and $A D C_{\text {Mean }}$ shows a substantial variety in different retrospective studies ${ }^{(14)}$; however, the majority of studies found a correlation. ${ }^{(13,16)}$ The correlation of $A D C_{\text {Mean }}$ is stronger and more uniform for parametrial involvement albeit in retrospective trials ${ }^{(13)}$; another research group showed that a combination of $\mathrm{ADC}_{\text {Mean }}$ and subjective analysis of the parametrium on $\mathrm{T} 2 \mathrm{~W}$ MRI increased AUC for predicting parametrial invasion. They used circle-like ROIs and recognized this to be a potential limitation of their retrospective study. In addition to this they did not assess interobserver variability. ${ }^{(12)}$ The relatively large proportion of IIB carcinoma in most studies hampers extrapolation to clinical practice, as these patients are generally treated with chemo-radiation therapy. Moreover, this patient selection could have increased the sensitivity of the test, whereas in our opinion, ADC would be of particular additional value in patients without clear parametrial invasion on T2W$\mathrm{MRI}$ and/or clinical examination. Woo et al. recently questioned the additional value of $A D C_{\text {Mean }}$ for the assessment of parametrial invasion when it was used as an adjunct to subjective T2W-MRI of the parametrium. However, because of the retrospective nature of their study, it might not have had sufficient power to justify subgroup analysis. ${ }^{(31)}$ They hypothesized that the correlation between $A D C_{\text {Mean }}$ and parametrial invasion was predominantly caused by MRI invisible tumors. We recognise that this might have influenced $A D C_{\text {Mean }}$ and therefore excluded MRI invisible tumors prior to analysis.

Our study has some limitations. DWI was performed on both 1.5 and 3T. Theoretically, major differences in ADC caused by differences in field strength are not expected. Ex vivo phantom models have indicated some small differences; however, in vivo literature indicates no significant differences in the ADC values of cervical cancer nor abdominal organs between 1.5 and 3T.(32) Our analyses showed no significant differences in $A D C_{\text {Mean }}$ between the 1.5 and $3 T$ groups, as supported by other research groups. ${ }^{(31)}$ Moreover, our study represents common general practice, where introduction of more advanced techniques and scanners is inevitable. Second, due to the introduction of new scanning protocols, imaging parameters varied to some extent. Current recommendations for DWI to optimize signal-to-noise ratio and decrease artefacts were followed. ${ }^{(33)}$ The changes in our protocol and the possible subsequent effect on $A D C$ values were previously evaluated by other research groups. ${ }^{(34-36)}$ These protocol changes had a small effect on the ADC value comparable with ADC variability as seen between vendors and single-system day-day repeatability (2,3-6,3\%). ${ }^{35)}$ However, as we assessed $\mathrm{ROI}$ measurement techniques within the same patient and protocol, these differences most likely have not affected these results. For the clinical data, the differences in field strength, scan protocol and B-value could have had some effect on the ADC values. However, these differences most likely have not have hampered our interpretation of the results. As there is little existing evidence for an optimal cutoff value, a predefined cut-off value was not defined prior to analysis of the dataset. However, for circle-like ROls, the cut-off values mentioned in all prior studies are comparable with our suggested cut-off: $0.85-0.90 \times 10^{-3} \mathrm{~mm} / \mathrm{s}$. For values within this 


\section{CONCLUSION}

This study shows that $A D C_{\text {Mean }}$ is an independent predictor of parametrial involvement. Single $\mathrm{ROI}$ measurements are a reliable method for determining $A D C_{\text {men }}$ in cervical cancer. Combining $A D C_{\text {Mean }}$ with T2W-MRI and clinical risk factors could result in more reliable pre-treatment staging, less adjuvant treatments, and therefore reduced patient burden and cost.
1. A. Baalbergen, Y. Veenstra, L. Stalpers, Primary surgery versus primary radiotherapy with or without chemotherapy for early adenocarcinoma of the uterine cervix, Cochrane Database Syst Rev (1) (2013) CD006248.

2. S.R. Salicru, J.F. de la Torre, A. Gil-Moreno, The surgical management of early-stage cervical cancer, Curr Opin Obstet Gynecol 25(4) (2013) 312-9.

3. M. Rotman, A. Sedlis, M.R. Piedmonte, B. Bundy, S.S. Lentz, L.I. Muderspach, R.J. Zaino, A phase III randomized trial of postoperative pelvic irradiation in Stage IB cervical carcinoma with poor prognostic features: follow-up of a gynecologic oncology group study, Int J Radiat Oncol Biol Phys 65(1) (2006) $169-76$.

4. K. Katanyoo, N. Praditsitthikorn, S. Tangjitgamol, S. Manusirivithaya, B. Supawattanabodee, Cost-utility analysis of treatments for stage IB cervical cancer, J Gynecol Oncol 25(2) (2014) 97-104

5. D. Cibula, R. Potter, F. Planchamp, E. Avall-Lundqvist, D. Fischerova, C. Haie Meder, C. Kohler, F. Landoni, S. Lax, J.C. Lindegaard, U. Mahantshetty, P. Mathevet, W.G. McCluggage, M. McCormack, R. Naik, R. Nout, S. Pignata, J. Ponce, D. Querleu, F. Raspagliesi, A. Rodolakis, K. Tamussino, P. Wimberger, M.R. Raspollini, The European Society of Gynaecological Oncology/European Society for Radiotherapy and Oncology/ European Society of Pathology guidelines for the management of patients with cervical cancer, Radiother Oncol 127(3) (2018) 404-416.

6. M.G. Thomeer, C. Gerestein, S. Spronk, H.C. van Doorn, E. van der Ham, M.G. Hunink, Clinical examination versus magnetic resonance imaging in the pretreatment staging of cervical carcinoma: systematic review and meta-analysis, Eur Radiol 23(7) (2013) 2005-18

7. S. Woo, C.H. Suh, S.Y. Kim, J.Y. Cho, S.H. Kim, Magnetic resonance imaging for detection of parametrial invasion in cervical cancer: An updated systematic review and meta-analysis of the literature between 2012 and 2016, Eur Radiol (2017)

8. J.Y. Lee, J. Youm, J.W. Kim, J.Y. Cho, M.A. Kim, T.H. Kim, D.H. Suh, M.C. Lim, N.H. Park, Y.S. Song, Identifying a low-risk group for parametrial involvement in microscopic Stage IB1 cervical cancer using criteria from ongoing studies and a new MRI criterion, BMC Cancer 15 (2015) 167.

9. C. Bourgioti, K. Chatoupis, A. Rodolakis, A. Antoniou, C. Tzavara, V. Koutoulidis, L.A. Moulopoulos, Incremental prognostic value of MRI in the staging of early cervical cancer: a prospective study and review of the literature, Clin Imaging 40(1) (2016) 72-8.

10. T.W. Kong, J. Kim, J.H. Son, S.W. Kang, J. Paek, M. Chun, S.J. Chang, H.S. Ryu, Preoperative nomogram for prediction of microscopic parametrial infiltration in patients with FIGO stage IB cervical cancer treated with radical hysterectomy, Gynecol Oncol 142(1) (2016) 109-14

11. S. Punwani, Diffusion weighted imaging of female pelvic cancers: concepts and clinical applications, Eur J Radiol 78(1) (2011) 21-9.

12. J.J. Park, C.K. Kim, S.Y. Park, B.K. Park, B. Kim, Value of diffusion-weighted imaging in predicting parametrial invasion in stage IA2-IIA cervical cancer, Eur Radiol 24(5) (2014) 1081-8. 
13. K. Nakamura, I. Joja, T. Nagasaka, C. Fukushima, T. Kusumoto, N. Seki, A. Hongo, J. Kodama, Y. Hiramatsu, The mean apparent diffusion coefficient value ( $A D C$ mean) on primary cervical cancer is a predictive marker for disease recurrence, Gynecol Oncol 127(3) (2012) 478-83.

14. F. Kuang, J. Ren, Q. Zhong, F. Liyuan, Y. Huan, Z. Chen, The value of apparent diffusion coefficient in the assessment of cervical cancer, Eur Radiol 23(4) (2013) 1050-8.

15. G. Zhou, X. Chen, F. Tang, J. Zhou, Y. Wang, Z. Wang, The Value of Diffusion-Weighted Imaging in Predicting the Prognosis of Stage IB-IIA Cervical Squamous Cell Carcinoma After Radical Hysterectomy, Int J Gynecol Cancer 26(2) (2016) 361-6.

16. M. Micco, H.A. Vargas, I.A. Burger, M.A. Kollmeier, D.A. Goldman, K.J. Park, N.R. Abu-Rustum, H. Hricak, E. Sala, Combined pre-treatment MRI and 18F-FDG PET/CT parameters as prognostic biomarkers in patients with cervical cancer, Eur J Radiol 83(7) (2014) 1169-76.

17. R.J. Karunya, P. Tharani, S. John, R.M. Kumar, S. Das, Role of Functional Magnetic Resonance Imaging Derived Parameters as Imaging Biomarkers and Correlation with Clinicopathological Features in Carcinoma of Uterine Cervix, J Clin Diagn Res 11(8) (2017) XC06-XC11.

18. S. Schob, H.J. Meyer, N. Pazaitis, D. Schramm, K. Bremicker, M. Exner, A.K. Hohn, N. Garnov, A. Surov, ADC Histogram Analysis of Cervical Cancer Aids Detecting Lymphatic Metastases-a Preliminary Study, Mol Imaging Biol (2017)

19. H. Bickel, K. Pinker, S. Polanec, H. Magometschnigg, G. Wengert, C. Spick, W. Bogner, Z. Bago-Horvath, T.H. Helbich, P. Baltzer, Diffusion-weighted imaging of breast lesions: Region-of-interest placement and different ADC parameters influence apparent diffusion coefficient values, Eur Radiol 27(5) (2017) 18831892

20. T. Tamada, C. Huang, J.M. Ream, M. Taffel, S.S. Taneja, A.B. Rosenkrantz, Apparent Diffusion Coefficient Values of Prostate Cancer: Comparison of 2D and 3D ROIs, AJR Am J Roentgenol 210(1) (2018) 113-117.

21. C. Inoue, S. Fujii, S. Kaneda, T. Fukunaga, T. Kaminou, J. Kigawa, T. Harada, T. Ogawa, Apparent diffusion coefficient (ADC) measurement in endometrial carcinoma: effect of region of interest methods on ADC values, J Magn Reson Imaging 40(1) (2014) 157-61.

22. D.M. Lambregts, G.L. Beets, M. Maas, L. Curvo-Semedo, A.G. Kessels, T. Thywissen, R.G. Beets-Tan, Tumour ADC measurements in rectal cancer: effect of ROI methods on ADC values and interobserver variability, Eur Radiol 21(12) (2011) 2567-74.

23. N. Mukuda, S. Fujii, C. Inoue, T. Fukunaga, Y. Tanabe, H. Itamochi, T. Ogawa, Apparent diffusion coefficient (ADC) measurement in ovarian tumor: Effect of region-of-interest methods on ADC values and diagnostic ability, J Magn Reson Imaging 43(3) (2016) 720-5.

24. E.R. DeLong, D.M. DeLong, D.L. Clarke-Pearson, Comparing the areas under two or more correlated receiver operating characteristic curves: a nonparametric approach, Biometrics 44(3) (1988) 837-45.

25. K. Downey, S.F. Riches, V.A. Morgan, S.L. Giles, A.D. Attygalle, T.E. Ind, D.P. Barton, J.H. Shepherd, N.M. deSouza, Relationship between imaging biomarkers of stage I cervical cancer and poor-prognosis histologic features: quantitative histogram analysis of diffusion-weighted MR images, AJR Am J Roentgenol 200(2) (2013) 314-20
26. Y. Guan, H. Shi, Y. Chen, S. Liu, W. Li, Z. Jiang, H. Wang, J. He, Z. Zhou, Y. Ge, Whole-Lesion Histogram Analysis of Apparent Diffusion Coefficient for the Assessment of Cervical Cancer, J Comput Assist Tomogr 40(2) (2016) 212-7.

27. Y. Lin, H. Li, Z. Chen, P. Ni, Q. Zhong, H. Huang, K. Sandrasegaran, Correlation of histogram analysis of apparent diffusion coefficient with uterine cervical pathologic finding, AJR Am J Roentgenol 204(5) (2015) 1125-31.

28. A. Gladwish, M. Milosevic, A. Fyles, J. Xie, J. Halankar, U. Metser, H. Jiang, N. Becker, W. Levin, L. Manchul, W. Foltz, K. Han, Association of Apparent Diffusion Coefficient with Disease Recurrence in Patients with Locally Advanced Cervical Cancer Treated with Radical Chemotherapy and Radiation Therapy, Radiology 279(1) (2016) 158-66

29. A.P. Gladwish, K. Han, W.D. Foltz, Variation in apparent diffusion coefficient measurements among women with locally advanced cervical cancer, Radiother Oncol 117(3) (2015) 532-5.

30. E. Giannotti, S. Waugh, L. Priba, Z. Davis, E. Crowe, S. Vinnicombe, Assessment and quantification of sources of variability in breast apparent diffusion coefficient (ADC) measurements at diffusion weighted imaging, Eur J Radiol 84(9) (2015) 1729-36.

31. S. Woo, S.Y. Kim, J.Y. Cho, S.H. Kim, Apparent diffusion coefficient for prediction of parametrial invasion in cervical cancer: a critical evaluation based on stratification to a Likert scale using T2-weighted imaging, Radiol Med (2017)

32. A.B. Rosenkrantz, M. Oei, J.S. Babb, B.E. Niver, B. Taouli, Diffusion-weighted imaging of the abdomen at 3.0 Tesla: image quality and apparent diffusion coefficient reproducibility compared with 1.5 Tesla, J Magn Reson Imaging 33(1) (2011) 128-35.

33. A.R. Padhani, G. Liu, D.M. Koh, TL. Chenevert, H.C. Thoeny, T. Takahara, A. Dzik-Jurasz, B.D. Ross, M. Van Cauteren, D. Collins, D.A. Hammoud, G.J. Rustin, B. Taouli, P.L. Choyke, Diffusion-weighted magnetic resonance imaging as a cancer biomarker: consensus and recommendations, Neoplasia 11(2) (2009) 102-25.

34. A. Celik, Effect of imaging parameters on the accuracy of apparent diffusion coefficient and optimization strategies, Diagn Interv Radiol 22(1) (2016) 101-7.

35. M.M. Jafar, A. Parsai, M.E. Miquel, Diffusion-weighted magnetic resonance imaging in cancer: Reported apparent diffusion coefficients, in-vitro and in-vivo reproducibility, World J Radiol 8(1) (2016) 21-49.

36. A. Wetter, F. Nensa, C. Lipponer, N. Guberina, T. Olbricht, M. Schenck, T.W. Schlosser, M. Gratz, T.C. Lauenstein, High and ultra-high b-value diffusion-weighted imaging in prostate cancer: a quantitative analysis, Acta Radiol 56(8) (2015) 1009-15. 


\section{PART II}

MR AND PET IMAGING FOR PREDICTING RESPONSE FOR LOCALLY ADVANCED CERVICAL CARCINOMA 


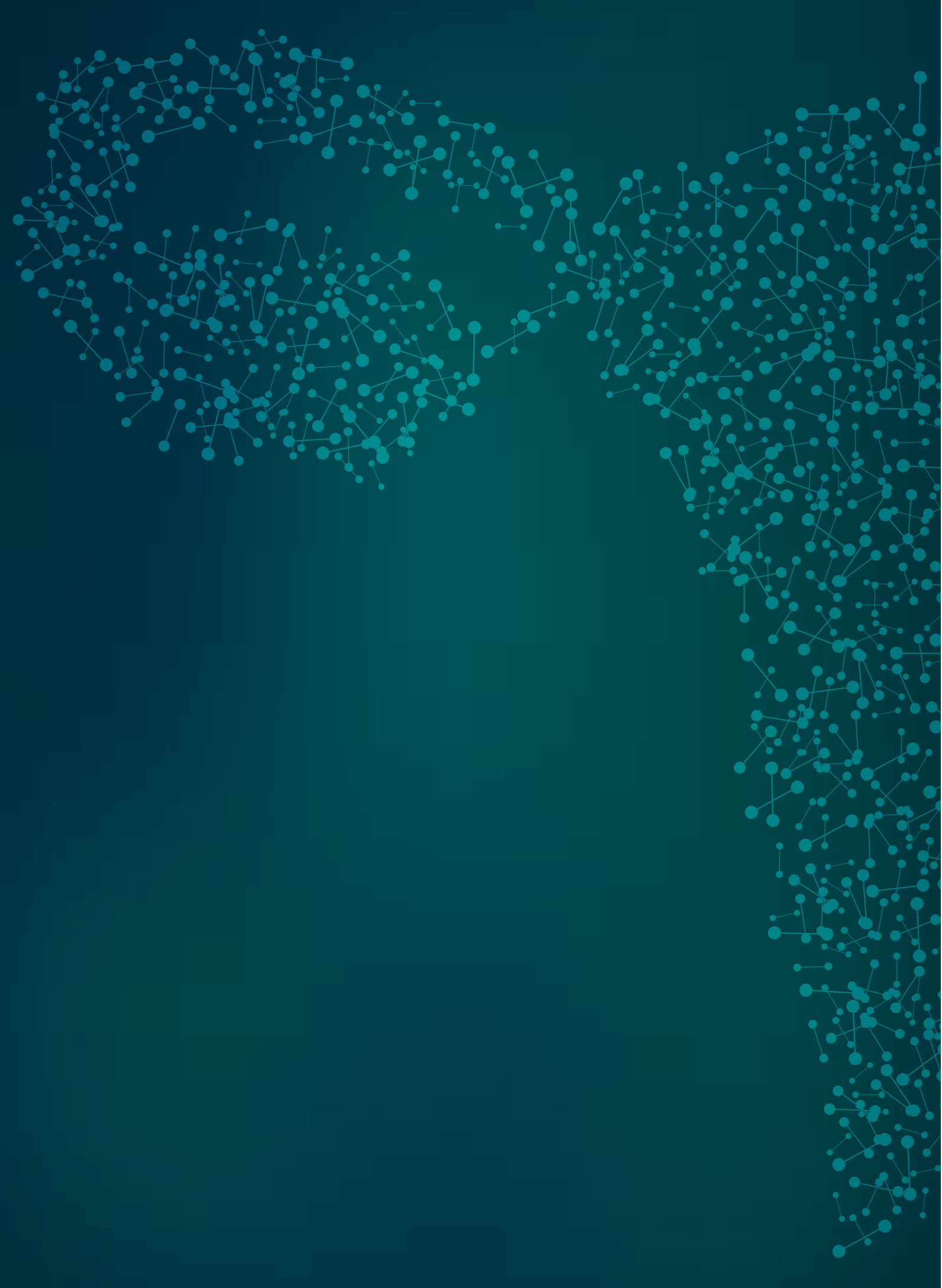

PREDICTIVE CRITERIA FOR MRI-BASED EVALUATION OF RESPONSE BOTH DURING AND AFTER RADIOTHERAPY FOR CERVICAL CANCER.

Mongula J

Slangen BFM,

Lambregts DMJ

Bakers FCH

Mahesh S

Lutgens LCHW

van Gorp T

Vliegen $\mathrm{R}$

Kruitwagen RFPM

Beets-Tan RGH

J Contemp Brachytherapy. 2016;8:181-8. 


\section{ABSTRACT}

\section{OBJECTIVE}

For cervical carcinoma, the presence of persistent disease after radiotherapy (RT) is a significant predictor for survival. To date, no standard protocol is available to evaluate response. This study was performed to assess MRI to evaluate presence of local residual disease during and after RT for FIGO stage IB1-IVA cervical cancer.

\section{METHODS}

Forty-two patients were included. Patients underwent MRI before external beam RT, at final intracavitary brachytherapy (BCT) and 2-3 months after completion of RT. Two blinded radiologists (observer 1: experienced/observer 2: less experienced) scored the likelihood of residual tumor. MRI was evaluated by means of (a) 'subjective' visual evaluation of T2 weighted MRI images and (b) 'objective' visual evaluation of T2 weighted MRI images according to predefined imaging criteria.

\section{RESULTS}

Seven patients had residual disease. Area under the receiver operating characteristics curve (AUC) for 'subjective' visual assessment was $0.79 / 0.75$ (observer 1/observer 2) after RT and 0.75/0.43 at final BCT. The combined 'objective' MRI criteria (isointense, nodular and irregular) resulted in improved prediction of residual tumor (AUCs of 0.91/0.85 after RT). For the less experienced observer, the MRI criteria set significantly improved prediction of residual tumor compared to 'subjective' visual assessment. Observer dependency decreased, kappa of 0.41 compared to 0.84 for the MRI criteria set after RT.

\section{CONCLUSION}

Compared to 'subjective' visual assessment, predefined 'objective' MRI criteria increase diagnostic performance and decrease observer dependency for assessing residual tumor after RT in cervical cancer.

\section{PURPOSE}

Cervical cancer is the third most common cancer worldwide and has an annual mortality rate of 270,000 . Approximately $30 \%$ of patients die due to persistent or recurrent disease after therapy. Recurrence mainly occurs during the first 2 years after treatment $\left(60-90 \%\right.$ of cases). ${ }^{(1-3)}$

The presence of persistent disease after radiotherapy is a significant predictor of patient survival. Although surgery has been suggested as an adjuvant therapy for cervical carcinoma patients after radiotherapy (RT), it is clear that it does not seem to improve overall survival. ${ }^{(4,5)}$

Moreover, the addition of salvage surgery to RT may increase both morbidity and cost. Therefore, salvage surgery should be reserved for selected cases with residual tumor. ${ }^{16}$. 7) To allow for such treatment stratification, it is necessary to accurately evaluate tumor responses after RT. However, no standard protocol for evaluating tumor response after RT is available. Gynecologic examination combined with biopsies several weeks after RT is often performed routinely. However, it is difficult to recognize small tumors within areas of radiation-induced fibrosis in suspicious cases. ${ }^{(4)}$ Furthermore, sampling errors may explain why, even with random biopsies, small microscopic tumor sites are easily missed, especially when suspected lesions are located outside the cervix or vagina. More accurate and (preferably) less invasive diagnostic modalities are required to allow a more reliable identification of patients who require salvage surgery to improve survival.

Magnetic resonance imaging (MRI) is often used to assess the responses of various tumors to RT including pelvic and cervical tumors. ${ }^{(8-12)}$ However, it is unclear which MR criteria are diagnostic for residual tumor tissue after RT. After treatment, the irradiated tumor bed often is replaced by fibrotic tissue. The problem with MRI is that differentiating fibrotic tissue from residual tumor tissue is difficult; for example, MRI of rectal cancer results in a false-positive rate of $50 \% .{ }^{(8,13)}$ The aims of the present study were to evaluate the ability of pelvic MRI to detect residual tumor after RT, to identify and validate objective imaging criteria predictive for residual tumor, and to assess their performance. 


\section{MATERIAL AND METHODS}

\section{PATIENTS}

Data for 42 patients with primary cervical cancer (International Federation of Gynecology and Obstetrics (FIGO) stage $\geq \mid \mathrm{b} 1$ ) who were referred to our gynecologic oncological center (Maastricht University Medical Center, Maastricht, The Netherlands) for radiation therapy between May 2004 and August 2010 were analyzed retrospectively. The inclusion criteria were as follows: (a) histologically-proven primary cervical carcinoma; (b) availability of pre- and post-treatment pelvic MRIs; and (c) treatment with external beam radiation therapy (EBRT) (46.0-50.4 Gy) and high-doserate brachytherapy $(B C T)$ with or without chemotherapy $(C T)$ or hyperthermia $(H T)$, Initially, BCT comprised 2-3 fractions for prescribing a total dose between 17-21 Gy. However, this has been replaced with MR-image-guided intra-cavitary plus interstitial BCT, which comprises 3-4 fractions of 7 Gy according to GEC-ESTRO guidelines. BCT was delivered over two or three sessions, with a 1 week interval between each session. The first BCT session was usually scheduled during the $5^{\text {th }}$ week of EBRT. Typically, the overall treatment time was $6-7$ weeks.

The baseline characteristics of the patients are listed in Table 1. Patients with distant metastasis at the time of presentation were excluded. The retrospective nature of the study meant that ethics board approval was not required (not subject to the Medical Research Involving Human Subjects Act).

MR

The majority of the MRI examinations (64\%) were performed using a 1.5-Tesla MRI unit (Intera Achieve; Philips Medical Systems, Best, The Netherlands; or Siemens Magnetom Avanto, Erlangen, Germany) and with a phased array surface coil. Patients were placed in a feet-first supine position. The imaging protocol comprised standard 2-dimensional T2W fast spin-echo images in three orthogonal directions (TR/TE (3200-3427)/(100-150 msec), 90-150 flip angle, 18-24 echo train length, 3-4 NSA, $0.98 \times 0.98 \times(3.00-5.00) \mathrm{mm}$ acquisition voxel size, 24-48 slices, 3.15-5.33 $\mathrm{min}$ acquisition time). The axial and coronal images were angled perpendicular and parallel to the cervical axis, respectively. The remaining MR examinations (36\%; $44 \%$ of which comprised examinations performed during BCT) were performed using a 3.0-Tesla MRI unit using a similar protocol (T2W FSE (TR/TE (7000/150), 28 echo train length, 2 NSA, $0.98 \times 0.98 \times(3.00-4.00) \mathrm{mm}$ acquisition voxel size, 40 slices, 3.15 min acquisition time). Patients received neither bowel preparation nor anti-spasmodic agents during the MRI examinations. MRI was performed at different time points: (a) before the onset of external beam radiation treatment; (b) immediately before the final BCT application; and (c) at a median 9 weeks (range 4-51 weeks) after the completion of radiotherapy.

\begin{tabular}{ll} 
TABLE 1. Baseline characteristics ( $\mathrm{n}=42)$ & \\
\hline Age median (range) & 53 years (31-81) \\
Histology & $33(79 \%)$ \\
Squamous cell carcinoma & $7(17 \%)$ \\
Adenocarcinoma & $2(4 \%)$ \\
Adenosquamous carcinoma & \\
FIGO & $2(5 \%)$ \\
I Ib1 & $6(14 \%)$ \\
II & $8(19 \%)$ \\
Ib1 & $17(40 \%)$ \\
Ib2 & $1(2 \%)$ \\
III & $4(10 \%)$ \\
Ib1 & \\
Ib2 & $4(10 \%)$ \\
IV & \\
IVa & $2(5 \%)$ \\
Therapy & $28(67 \%)$ \\
Radiotherapy & $12(28 \%)$ \\
Chemo-radiotherapy & 9 weeks (4-51) \\
Hyperthermia-radiotherapy & 24 months (13-81) \\
Time between last radiotherapy and MRI Median (range) \\
Follow up Median (range)
\end{tabular}

FIGO - International Federation of Gynecology and Obstetrics, MRI - Magnetic Resonance Imaging

\section{IMAGE EVALUATION}

The MR images were independently scored by a senior radiologist (FB; reader 1 ) and a junior radiologist (SM; reader 2), who have 7 and 2 years, respectively, experience in pelvic MR imaging. The two readers were only aware of the initial FIGO stage, but were blinded to each other's interpretation of the results and the follow-up data. First, the readers were asked to assess the presence of residual tumor based on a 'subjective' visual assessment of the T2W images using the following confidence level scores: 0 , definitely no residual tumor; 1 , probably no residual tumor; 2 , possibly residual tumor: 3 , probably residual tumor; 4 , definitely residual tumor. The readers were not given any instructions or asked to search for certain criteria, and were free to interpreted the scans based on prior experience. Subsequently, both readers were asked to evaluate each of the following imaging criteria (using a five-point confidence level score: (a) the presence of an isointense mass (isointense compared with the initial tumor [mostly 
hyperintense lesions at the time of presentation]): (b) the presence of a hypointense (fibrotic) mass; (c) the shape of the tumor (nodular or non-nodular); (d) the aspect of the border of the cervix (regular or irregular); (e) signal homogeneity or heterogeneity; and (f) the shape of the cervix (normal or deformed). The different imaging criteria are shown in Figure 1. All interpretations were performed using MR images obtained after radiotherapy and, if available $(n=29 / 42)$, on MR images obtained immediately before the final BCT application. The pre-treatment images were at the reader's disposal. If MRI was performed immediately before the final BCT, these images were also at the disposal of the reader. The readers were blinded to all other clinical outcomes and imaging.

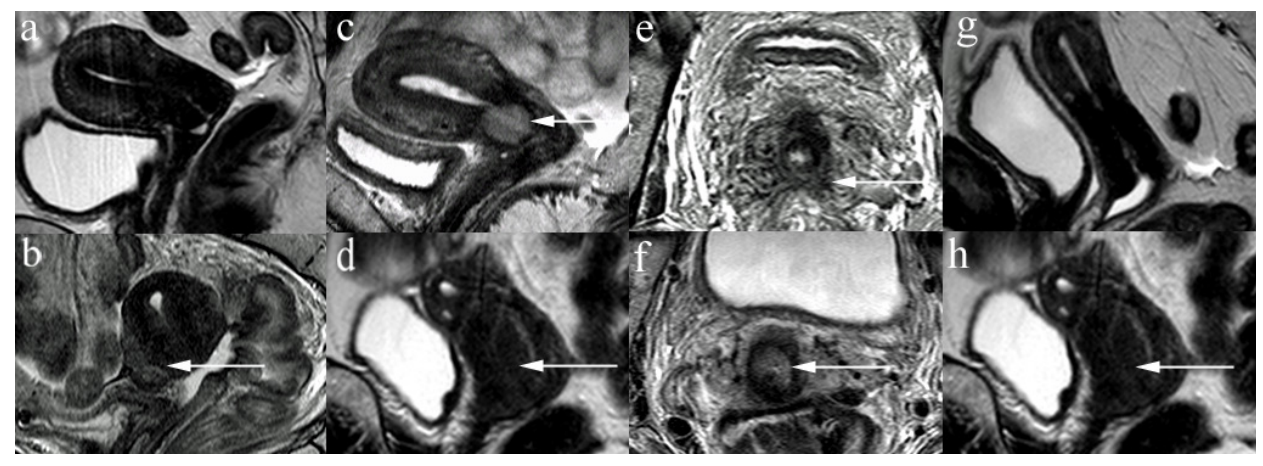

FIGURE 1. Sagittal (a-d, g-h) and axial (e-f) T2-weighted images of different patients with a cervical carcinoma after radiotherapy.

The patients in $\mathbf{a}, \mathbf{d}, \mathbf{e}, \mathbf{g}$ and $\mathbf{h}$ had no residual tumor. To the contrary: the patients in $\mathbf{b}, \mathbf{c}$ and $\mathbf{f}$ showed residual tumor. The images illustrate the imaging criteria used to assess the presence of residual tumor. $\mathbf{a} / \mathbf{b}$. The first criteria was the signal intensity of the cervix after treatment, which was scored as hypointense without any signs of an isointense tumor mass (a) or as a persistent isointense signal intensity area, indicative of residual tumor (white arrow in $\mathbf{b}$ ). $\mathbf{c} / \mathbf{d}$. The second criterion was the homogeneity of the signal intensity in the cervix, which was scored as either homogeneous (like the homogeneously isointense lesion indicated by the white arrow in c) or heterogeneous, like the partly isointense and partly hypointense lesion indicated by the arrow in $\mathrm{d}$. e/f. The third and fourth criterion were the aspect of the cervical border and shape (nodular vs non-nodular) of a (partly) isointense laesion. The cervical border was scored as irregular (e) or regular (f). The shape was scored as either non-nodular (e) or nodular (f) $g / h$. The final criterion was the presence or absence of persistent deformation of the cervix after treatment. The cervical shape was scored either as normalized ( $\mathbf{g})$ or persistently deformed $(\mathbf{h})$.

\section{STANDARD OF REFERENCE}

The presence or absence of local residual tumor (in the cervix and/or vagina, parametrium, bladder, and rectum) was determined by: (1) histopathology after surgical resection ( $n=6)$; or (2) post-treatment gynecologic examination (under anesthesia) 3 months after the completion of RT, either with $(n=21)$ or without a biopsy (not clinically indicated $\mathrm{n}=15$ patients), combined with at least 12 months documented follow-up. Follow-up comprised a gynecologic examination every 3 months, which was conducted by a gynecologic or radiation oncologist. Local residual tumor and/or metastasis was defined as the presence of residual tumor (identified pathologically) or a growing mass on consecutive images, along with an increase in the level of tumor markers in the serum (Squamous-cell carcinoma (SCC) or Carcinoembryonic antigen CEA). (14)

\section{STATISTICAL ANALYSIS}

Statistical analyses were performed using SPSS Statistics, v18.0 (SPSS Inc, Chicago, Ill.) and Stata v11.0 (StataCorp LP, Texas) software. Interobserver variations were assessed by means of weighted kappa statistics, with quadratic kappa weighting. Each of the $\mathrm{MRI}$ criteria were compared and then combined to determine the best combination. Receiver operating characteristics (ROC) curves were constructed to evaluate diagnostic performance for (a) the 'subjective' T2W visual response assessment, (b) the 'objective' imaging criteria, and (c) the combined MRI criteria. The areas under the ROC curve (AUC), sensitivity, specificity, and positive- and negative-predictive value were calculated. At the beginning of the study, the decision was made to dichotomize the confidence level scores between 2 and 3 to yield a relatively high sensitivity for residual tumor. The sensitivity and specificity of the different scoring methods were compared using the Mcnemar test (for paired data) or the Chi-squared test (for unpaired data). The AUCs were compared using to the method described by De Long et al. P values $<0.05$ were considered statistically significant. ${ }^{115}$ 


\section{RESULTS}

\section{PATIENT AND TREATMENT CHARACTERISTICS}

After radiation treatment, seven patients still had local residual tumor; five of these patients also had concurrent distant metastases. Eight patients had distant metastases only, median time to metastases 15 months (range 3-31 months). Twenty-seven patients had no evidence of disease after a median follow-up of 24 months (range 1381 months). The two patients with local residual tumor but without distant metastasis underwent salvage surgery.

\section{DIAGNOSTIC PERFORMANCE FOR ASSESSMENT OF RESPONSE}

Figure 2 and Tables 2 and 3 show the diagnostic performance of both readers based on the 'subjective' visual response, the 'objective' imaging criteria, and the combined MRI criteria. The interobserver agreement for the individual imaging criteria is shown in Tables 2, 3 and 4

\section{'SUBJECTIVE' VISUAL ASSESSMENT AFTER COMPLETION OF RT}

For reader 1 (the more experienced), the 'subjective' visual assessment after radiotherapy resulted in an AUC of 0.79 , sensitivity of $71 \%$, and specificity of $86 \%$. For the junior reader (reader 2), the results were $0.75,86 \%$, and $46 \%$, respectively. Interobserver agreement for the 'subjective' visual response assessment after treatment was moderate $(\mathrm{K}=$ 0.41). In 29 patients, additional MR images taken immediately before the final BCT application were available and at the disposal of the reader. Using this MRI, the AUC for reader 1 regarding assessment of the tumor response after completion of RT was 0.99. In the group with 13 patients who did not have this MRI, the AUC was $0.57(p=0.02)$. The corresponding AUCs for reader 2 were 0.90 and $0.60(p=0.08)$.

\section{'OBJECTIVE' IMAGING CRITERIA AFTER COMPLETION OF RT}

A hypointense mass and signal homogeneity showed a low AUC 0.49-0.63. An isointense mass, a nodular shape, and an irregular border yielded AUCs of 0.79, 0.790.83 , and $0.82-0.87$, respectively, when each was used as a stand-alone criterion. Combining all three criteria yielded an AUC of 0.85-0.91. For both readers, using the combined criteria resulted in an AUC higher than that obtained when using 'subjective' visual assessment (AUC 0.91 vs. AUC 0.79, p=0.15 for reader 1; and AUC 0.85 vs. AUC $0.75, p=0.02$ for reader 2 ). For the less experienced observer, the specificity improved from $46 \%$ to $80 \%(p<0.01)$. Interobserver agreement regarding the individual imaging criteria ranged from $\mathrm{k}=0.12$ to $\mathrm{k}=0.91$. The $\mathrm{k}$ value for abnormal cervical shape was moderate (0.41). The $\mathrm{k}$ value for the combined criteria set (isointense mass, a nodular
As shown in table 4 there were no major, nor substantle differences between both observers for the MR criteria set. Both moderate differences correspond to two cases with no evidence of local residual disease after follow-up in which the less experienced reader scored an isointense mass in contrast to the experienced reader.

\section{RESPONSE ASSESSMENT DURING BRACHYTHERAPY}

MR images taken immediately before the final BCT were available for 29/42 patients. The 'subjective' (visual) assessment of MR images taken immediately before the final $B C T$ resulted in an AUC of 0.75 , sensitivity of $75 \%$, and specificity of $36 \%$ for reader 1 , and $0.43,100 \%$, and $8 \%$, respectively, for reader 2 . Each of the three 'objective' imaging criteria on images taken immediately before the final BCT resulted in AUCs of 0.51-0.82. Only abnormal shape and a hypointense mass showed a higher AUC than the subjective assessment when used as stand-alone criteria. Interobserver agreement was rather low $(0.29$ and 0.46$)$. Only the $\kappa$ value for isointense mass $(0.88)$ reached an acceptable level.

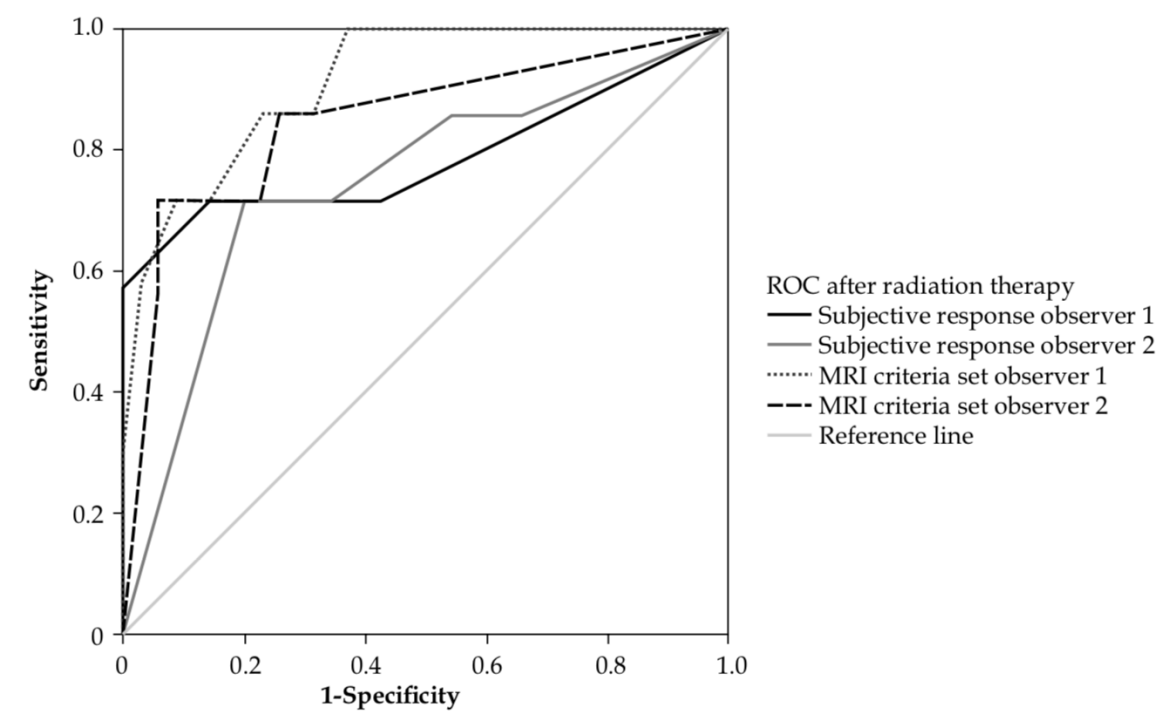

FIGURE 2. Reciever operating curve for magnetic resonance imaging (MRI) analysis after radiotherapy, observer 1 and 2 

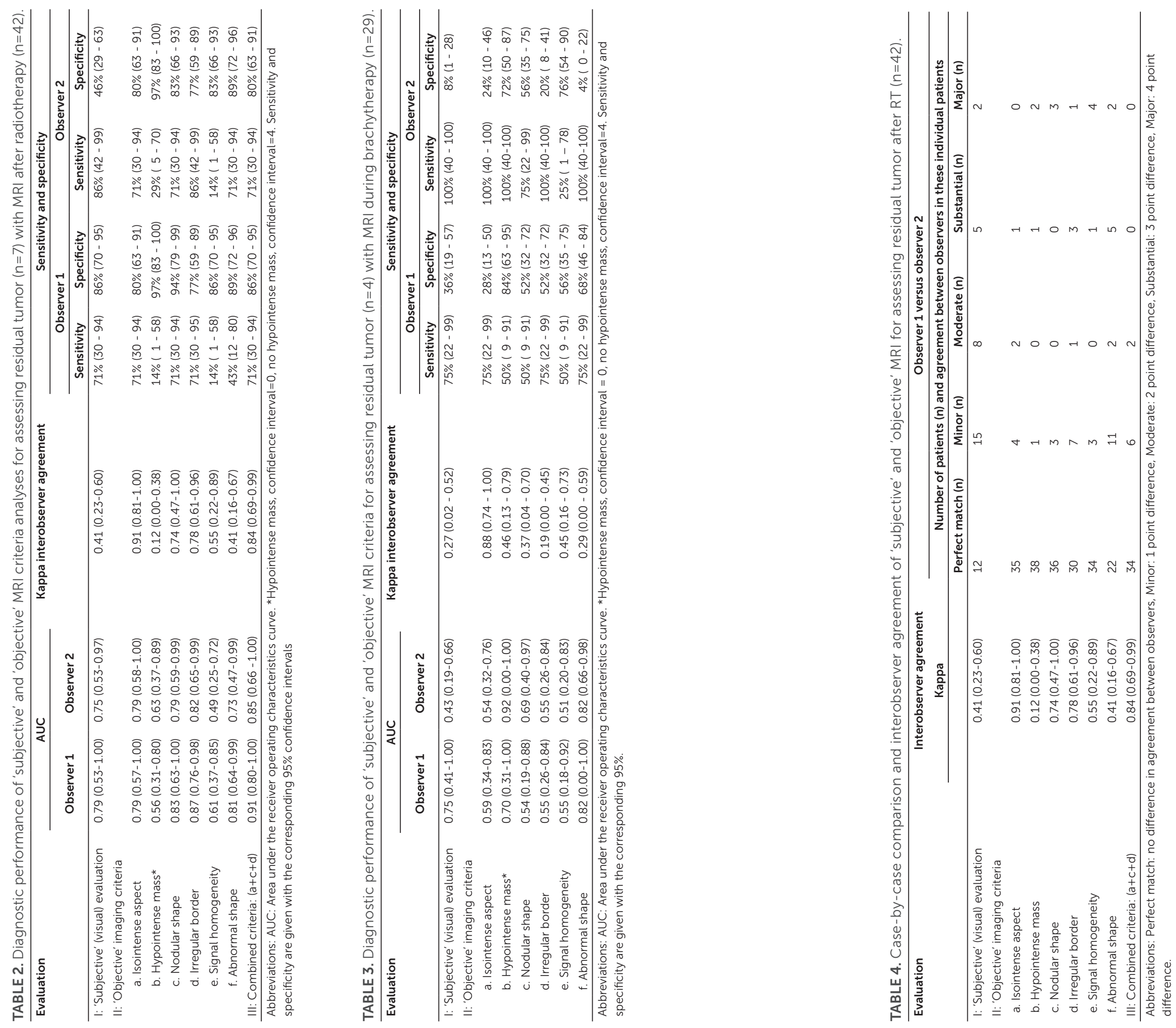


\section{DISCUSSION}

The present study examined the utility of MRI performed during and after RT (either with or without $\mathrm{CT}$ or $\mathrm{HT}$ ) for detecting residual tumor. We also attempted to identify imaging criteria predictive for residual tumor and assessed their accuracy.

'Subjective' visual assessment of residual disease on post-RT MRI resulted in a reasonable AUC of 0.79 for the more experienced reader and an AUC of 0.75 for the junior reader. The interobserver agreement was poor. However, the use of combined 'objective' criteria (isointense mass, nodular shape, and irregular border) to assess residual disease after RT led to a much better performance, and increased the specificity parameter for the less experienced reader. Moreover, the interobserver agreement improved to excellent when the assessment was performed using three 'objective' criteria.

This is the first report focusing on observer performance when interpreting MRI scans with the aim of identifying residual disease after RT. The results show that using 'objective' criteria improved the performance of both readers. This is due to the introduction of a systematic scoring system, in which certain criteria have to be scored separately. Experienced readers may unconsciously use similar criteria; this may explain why the predictive value for the less expert reader improved to a greater extent than that of the more experienced reader when using the objective criteria rather than the subjective assessment method. However, the experienced reader also improved when using the objective criteria.

The MRI criteria were derived from the literature in conjunction with an independent expert pelvic radiologist. Morphologic visual T2W assessment of cervical tumors is often used to evaluate MR imaging of residual disease after RT. ${ }^{(16)}$ Initial studies reported different values for diagnostic accuracy (sensitivity $80-100 \%$ and specificity 56-100\%). ${ }^{(9-12)}$ Vincens et al. reported an equivocal diagnosis in 16/44 cases when iso-/hyperintensity combined with contrast enhancement were used as 'objective' MRI criteria; subjective assessment led to an equivocal diagnosis in only 5/44 cases. (12) However, specificity was still low (55\%) for the group with a definite diagnosis, indicating that isointensity is inaccurate when used as a single MRI criterion. In our data we found that isointensity showed a higher specificity (80\%). This difference may be explained by the fact that Vincens et al. assessed treatment response at 3-8 weeks after the completion of RT, whereas we assessed the response at a median 9 weeks. Thus, the results of Vincens et al. may have been affected by early radiation effects, with granulomatous tissue formed in the acute post-RT phase mimicking residual tumor isointensity on MRI, resulting in many more false-positives. This hypothesis is supported by the fact that we observed a similarly low specificity for isointensity on MRI performed immediately before the completion of the final BCT.

We found it interesting that both readers performed better when visually assessing the post-RT response of patients for whom an additional scan was performed immediately before the last $\mathrm{BCT}$. The expert reader in particular increased his diagnostic performance. This suggests that the readers found the additional information provided by the BCT MR images very useful during their final assessment of the treatment response. This can be explained by the fact that volume regression over time can be observed more accurately on consecutive scans. This phenomenon was suggested by Mayr and Wang et al., who found volume regression analyses after EBRT showed a relatively high diagnostic performance. ${ }^{177}$

The prevalence of recurrent/persistent local tumor in our dataset (17\%) is rather low when compared to that reported in the literature. This may have led to an underestimation of the positive-predictive value. The low recurrence rate may be due to several reasons. First, due to our inclusion criteria patients who did not finish the imaging or treatment protocols due to the presence of early progressive disease were excluded; $38 \%$ of the current study population had tumors at FIGO stage Ila or less. Second, almost all patients received concurrent CT or HT treatment in addition to RT, which has a higher cure rate than RT alone. ${ }^{(18,19)}$ Third, a substantial proportion of our treatment group received MRI-based BCT. MRI-based BCT is superior to 2D-based radiotherapy with respect to local relapse rates for cervical carcinomas measuring > $5 \mathrm{~cm}$. ${ }^{(20-23)}$

The present study had several limitations. First, the absence of residual disease was not confirmed histopathologically in all cases. This was because not all patients underwent surgery. However, we believe that the absence of recurrence at long-term follow-up is a good indicator of a lack of residual disease. Moreover, when surgery is performed early after RT, microscopic tumors may still be present (RT may not have exerted its full effect at this time). Other research groups tried to solve this problem by including patients with microscopic tumors $(<1 \mathrm{~cm})$ in the complete response group. However, this could lead to an underestimation of the number of patients with recurrent tumor as these foci can still progress to a recurrence. A second limitation is the small number of patients examined. Specifically, the number of patients that underwent MRI during brachytherapy was low; this is because we only started performing MRI-based BCT at 
our center in 2008. A third limitation is the retrospective nature of the study, which meant that different imaging protocols, different MRI scanners, and different magnetic fields were used. This may have affected the results. However, because $71 \%$ of all postand pre-treatment scans were performed using a 1.5 Tesla MRI scanner, and all imaging protocols were 'routine clinical protocols', we believe that these differences would not have had a significant effect on the final results. Indeed, the data are representative of those obtained during daily clinical practice.

\section{CONCLUSION}

The use of predefined 'objective' MRI criteria for assessing residual tumor after RT increases diagnostic performance and makes an accurate diagnosis less dependent upon observer experience. The availability of a brachytherapy MRI scan during treatment improves the detection of residual cervical cancers on post-treatment MRI scans. With the increasing use of MRI during brachytherapy, in future prospective studies, this finding should be investigated more accurately in order to tailor treatment for the patient.

\section{REFERENCE}

1. Kitchener HC, Hoskins W, Small W, Jr., et al. The development of priority cervical cancer trials: a Gynecologic Cancer InterGroup report. Int J Gynecol Cancer. 2010;20(6):1092-100

2. Babar S, Rockall A, Goode A, et al. Magnetic resonance imaging appearances of recurrent cervical carcinoma. Int J Gynecol Cancer. 2007;17(3):637-45.

3. Elit L, Fyles AW, Devries MC, et al. Follow-up for women after treatment for cervical cancer: a systematic review. Gynecologic oncology. 2009;114(3):528-35.

4. Nijhuis ER, van der Zee AG, in 't Hout BA, et al. Gynecologic examination and cervical biopsies after (chemo) radiation for cervical cancer to identify patients eligible for salvage surgery. Int J Radiat Oncol Biol Phys. 2006;66(3):699-705.

5. Keys HM, Bundy BN, Stehman FB, et al. Radiation therapy with and without extrafascial hysterectomy for bulky stage IB cervical carcinoma: a randomized trial of the Gynecologic Oncology Group. Gynecologic oncology. 2003;89(3):343-53

6. Hong JH, Tsai CS, Lai CH, et al. Recurrent squamous cell carcinoma of cervix after definitive radiotherapy. Int J Radiat Oncol Biol Phys. 2004;60(1):249-57.

7. Houvenaeghel G, Lelievre L, Buttarelli M, et al. Contribution of surgery in patients with bulky residual disease after chemoradiation for advanced cervical carcinoma. European journal of surgical oncology. 2007; 33(4):498-503.

8. Dresen RC, Beets GL, Rutten HJ, et al. Locally advanced rectal cancer: MR imaging for restaging after neoadjuvant radiation therapy with concomitant chemotherapy. Part I. Are we able to predict tumor confined to the rectal wall? Radiology. 2009;252(1):71-80

9. Hricak $\mathrm{H}$, Lacey $C G$, Sandles $L G$, et al. Invasive cervical carcinoma: comparison of $M R$ imaging and surgical findings. Radiology. 1988;166(3):623-31.

10. Hatano K, Sekiya Y, Araki H, et al. Evaluation of the therapeutic effect of radiotherapy on cervical cancer using magnetic resonance imaging. Int J Radiat Oncol Biol Phys. 1999;45(3):639-44

11. Manfredi R, Maresca G, Smaniotto D, et al. Cervical cancer response to neoadjuvant therapy: MR imaging assessment. Radiology. 1998;209(3):819-24.

12. Vincens $E$, Balleyguier $C$, Rey $A$, et al. Accuracy of magnetic resonance imaging in predicting residual disease in patients treated for stage IB2/II cervical carcinoma with chemoradiation therapy : correlation of radiologic findings with surgicopathologic results. Cancer. 2008;113(8):2158-65.

13. Vliegen RF, Beets $\mathrm{GL}$, Lammering $\mathrm{G}$, et al. Mesorectal fascia invasion after neoadjuvant chemotherapy and radiation therapy for locally advanced rectal cancer: accuracy of MR imaging for prediction. Radiology. 2008;246(2):454-62

14. Forni F, Ferrandina G, Deodato F, et al. Squamous cell carcinoma antigen in follow-up of cervical cancer treated with radiotherapy: evaluation of cost-effectiveness. Int J Radiat Oncol Biol Phys. 2007;69(4):1145-

15. DeLong ER, DeLong DM, Clarke-Pearson DL. Comparing the areas under two or more correlated receiver

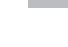
(1)

r

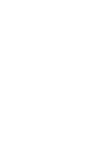


16. Jeong YY, Kang HK, Chung TW, et al. Uterine cervical carcinoma after therapy: CT and MR imaging findings. Radiographics. 2003;23(4):969-81; discussion 81.

17. Wang JZ, Mayr NA, Zhang D, et al. Sequential magnetic resonance imaging of cervical cancer: the predictive value of absolute tumor volume and regression ratio measured before, during, and after radiation therapy. Cancer. 2010;116(21):5093-101.

18. (CCCMAC). CfCCM-aC. Reducing uncertainties about the effects of chemoradiotherapy for cervical cancer: individual patient data meta-analysis. Cochrane Database Syst Rev. 2010; Jan 20;(1).

19. Lutgens $L$, van der Zee J, Pijls-Johannesma $M$, et al. Combined use of hyperthermia and radiation therapy for treating locally advanced cervix carcinoma. Cochrane Database Syst Rev. 2010(3):CD006377.

20. Potter R, Dimopoulos J, Georg P, et al. Clinical impact of MRI assisted dose volume adaptation and dose escalation in brachytherapy of locally advanced cervix cancer. Radiotherapy and oncology. 2007;83(2):148-55.

21. Dimopoulos JC, Petrow P, Tanderup K, et al. Recommendations from Gynaecological (GYN) GEC-ESTRO Working Group (IV): Basic principles and parameters for MR imaging within the frame of image based adaptive cervix cancer brachytherapy. Radiotherapy and oncology 2012.

22. Potter R, Georg P, Dimopoulos JC, et al. Clinical outcome of protocol based image (MRI) guided adaptive brachytherapy combined with 3D conformal radiotherapy with or without chemotherapy in patients with locally advanced cervical cancer. Radiotherapy and oncology. 2011;100(1):116-23.

23. Owrangi AM, Prisciandaro Jl, Soliman A, et al. Magnetic resonance imaging-guided brachytherapy for cervical cancer: initiating a program. J Contemp Brachytherapy. 2015;7(5):417-22. 


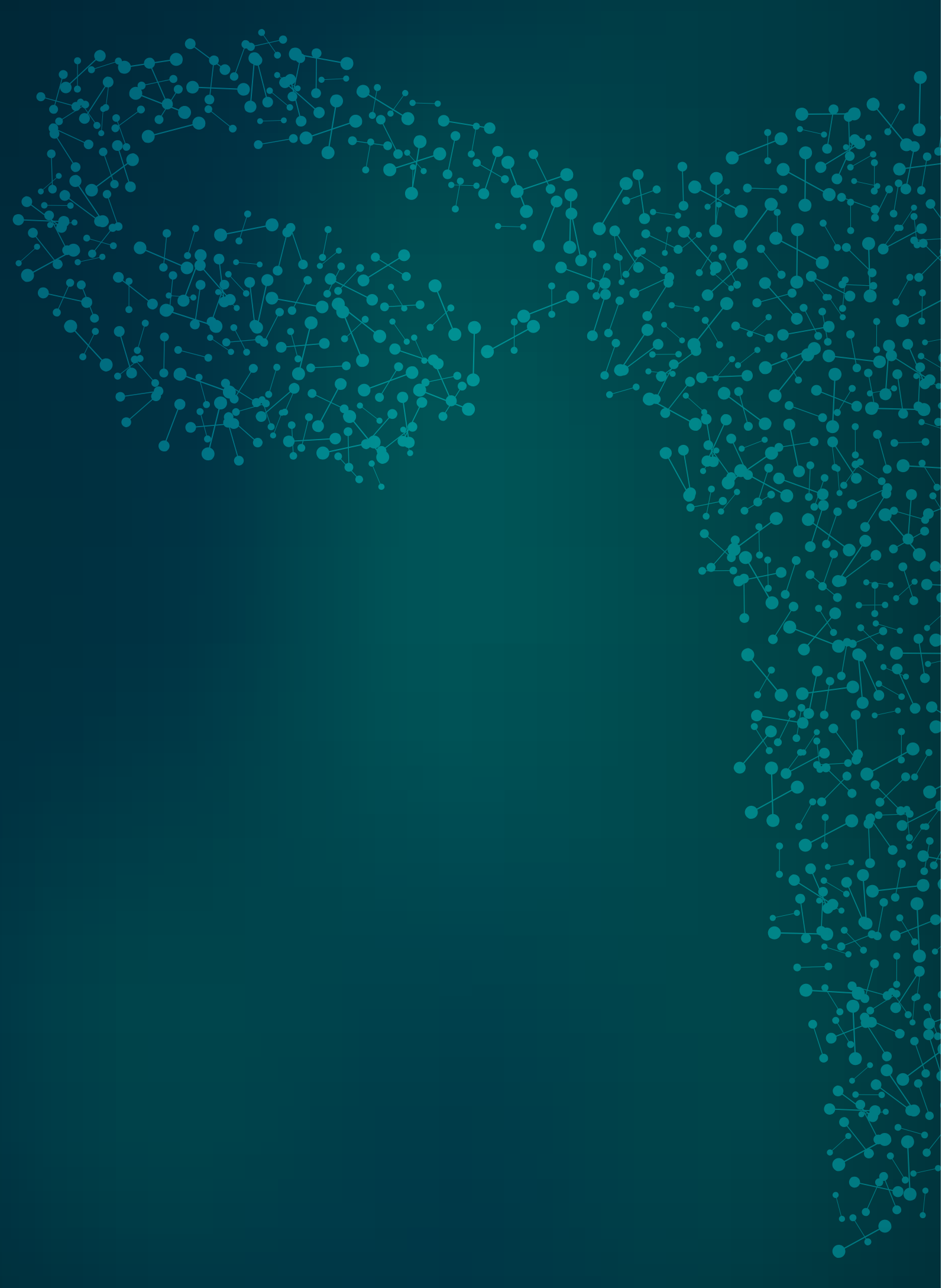

CONSECUTIVE MAGNETIC RESONANCE IMAGING DURING BRACHYTHERAPY FOR CERVICAL

CARCINOMA: PREDICTIVE VALUE OF VOLUME MEASUREMENTS WITH RESPECT TO PERSISTENT DISEASE AND PROGNOSIS.

Mongula JE

Slangen BFM

Lambregts DMJ

Cellini F

Bakers FCH

Lutgens LCHW

Van Gorp T

Kruse A

Kruitwagen RFPM

Beets-Tan RGH

Radiat Oncol. 2015;10:252. 


\section{ABSTRACT}

\section{BACKGROUND}

Cervical cancer is associated with a high yearly mortality. The presence of persistent disease after radiotherapy is a significant predictor of patient survival. The aim of our study was to assess if tumor volume regression measured with MR imaging at the time of brachytherapy can discriminate between patients who eventually will achieve a complete response to radiotherapy from those who will not. The second objective was to evaluate whether tumor volume regression predicts overall treatment failure.

\section{METHODS}

MRI was evaluated quantitatively in 35 patients; by means of tumor volumetry on T2weighted MR images before treatment, at the first BCT application, and at the final BCT. The MR images were independently analyzed by two investigators. As a reference, standard histopathologic confirmation of residual tumor and/or clinical exam during follow-up $>1$ year were used. Area under the curve were compared, P-values $<0.05$ were considered significant.

\section{$5 \quad$ RESULTS}

There was a good correlation between volume measurements made by the two observers. A residual tumor volume $>9.4 \mathrm{~cm}^{3}$ at final $\mathrm{BCT}$ and tumor volume regression $<77 \%$ of the pre-treatment volume were significantly associated with local residual tumor after completion of therapy $\left(p<0.02\right.$ ) (AUC, $0.98-1.00$ ). A volume $>2.8 \mathrm{~cm}^{3}$ at final BCT was associated with overall treatment failure $(p<0.03)$

\section{CONCLUSION}

Our study shows that volume analysis during BCT is a predictive tool for local tumor response and overall treatment outcome. The potential of local response assessment to identify patients at high risk of overall treatment failure is promising.

\section{BACKGROUND}

Cervical cancer is associated with a yearly mortality of 270.000 patients. ${ }^{(1-3)}$ The presence of a persistent cervical carcinoma after radiotherapy is a significant predictor of patient survival. In a select group of patients with persistent tumors after radiotherapy, surgical resection can be beneficial. ${ }^{(4,5)}$

The detection of residual tumors by gynecological examination, MRI, and PET-CT is complicated by the occurrence of false positive results and interobserver dependency, which are mainly due to post-radiation induced fibrosis. Diagnoses based on histological biopsies are limited by the occurrence of false negative results. ${ }^{(6,7,8,9)}$ It has been reported that tumor volume assessment on repeated MRIs during radiotherapy treatment can be used to predict tumor response to radiotherapy. ${ }^{(10-13)}$ In the past decade a new concept was clinically introduced by the GEC-ESTRO working group taking the tumor regression during external beam radiation into account for treatment planning with brachytherapy (BCT). ${ }^{(14)}$ Applying this concept, i.e. MR-image guided adaptive brachytherapy (IGABT), assessing the residual tumor volume and/or target volume at high risk at the time of BCT has been demonstrated to improve outcome significantly in locally advanced cervix carcinoma.(14-16) For optimal assessment of residual tumor burden at the time of $\mathrm{BCT}$ T2-weighted MR images are obtained with the BCT applicator in place. This imaging sequence results in the best quality to discriminate tumor from cervical stroma. Applicator based distortions and artefacts are acceptable for T2W sequences using MR-compatible applicators. ${ }^{(17)}$

Until now, volumetric analysis for predicting treatment response on MRI used in this setting, with the BCT applicator in place, has never been used. If MR-based tumor volume assessment at the time of BCT is a reliable option, it could be used to predict tumor response both local and for overall outcome.

The aim of our study was to assess if absolute tumor volume and tumor regression at the time of BCT can discriminate between patients who eventually achieve a complete response from those who do not. The second objective was to evaluate whether local volume regression can predict overall treatment failure. 


\section{METHODS AND MATERIALS}

\section{PATIENTS}

We performed a retrospective analysis of patients with primary cervical cancer (International Federation of Gynecology and Obstetrics (FIGO) stages $\geq \mathrm{IB}$ ), who were referred to our centre (Maastricht University Medical Center en MAASTRO clinic) between March 2008 and August 2010 for radiotherapy. Inclusion criteria consisted of: (1) a histologically proven primary cervical carcinoma; (2) availability of MRI images before treatment (a), after EBRT at the first BCT application (b) and at the last BCT treatment (c); (3) treatment consisting of EBRT (46.0-50.4 Gy and high-dose-rate $\mathrm{BCT}$, with or without concomitant chemotherapy (CT) or hyperthermia (HT); and (4) absence of distant metastasis at diagnosis.

\section{RADIOTHERAPY PROTOCOL}

For external beam radiation (EBRT) a dose of 45-50.4 Gy was delivered in 25-28 daily fractions, 5 days a week. Dose specification and homogeneity requirements were according to ICRU-50. In case of concomitant chemotherapy or regional hyperthermia, these modalities were applied once-weekly during EBRT. The first BCT was scheduled in the final week of EBRT of following EBRT. For brachytherapy MR-

compatible applicators (Nucletron) were used placing an intra-uterine tandem and vaginal ovoids with or without the use of interstitial needles. For BCT planning the GEC-ESTRO guidelines were followed, contouring the gross tumor, a high-risk (HR) and intermediate risk clinical target volume (IR-CTV) and the organs at risk. Typically 3 applicator insertions were performed under general anesthesia with one week interval. For the second $\mathrm{BCT}$ procedure the applicator was left in place for one night allowing $2 \mathrm{BCT}$ treatments with an interval of at least $15 \mathrm{hrs}$. Hence, $4 \mathrm{BCT}$ treatments were delivered in 2 weeks. A dose of 7 Gy per fraction was aimed to prescribe to the HRCTV, aiming to deliver a $D 90 \geq 85$ Gy.

\section{MRI PROTOCOL}

MRI was performed with a 1.5-Tesla MRI unit (Intera (Achieva); Philips Healthcare, Best, The Netherlands or Siemens Magnetom Avanto, Erlangen, Germany) in 52\% of the studies and with a 3.0-Tesla MRI unit (Achieva 3.0TX; Philips Healthcare, Best, the Netherlands) in $48 \%$ of the studies. Patients were placed in a feet-first supine position. The protocol consisted of 2D T2-weighted (T2W) fast spin-echo images (TR/TE 3200-

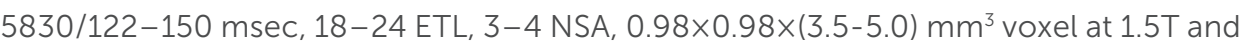
TR/TE 7000/150, 28 ETL, 2 NSA, $0.98 \times 0.98 \times(3.00-4.00) \mathrm{mm}^{3}$ voxel at 3 T). Scans were made in three planes (sagittal, axial, and coronal), while the axial and coronal planes

were angled perpendicular and parallel to the cervical axis, respectively. Patients did not receive bowel preparation or anti-spasmodic agents. MRI was performed at different time points: before treatment (a), after EBRT at the first BCT application (b), and at the final BCT treatment (c). All MRI scans (except the first one, before the onset of radiotherapy) were performed immediately before the $\mathrm{BCT}$ fractions were given but with the BCT applicator in situ.

\section{IMAGE EVALUATION}

MRI was evaluated quantitatively; by means of tumor volumetry on T2-weighted MR images before treatment (a), after EBRT at the first BCT application (b), and at the final $\mathrm{BCT}$ treatment (c)

The MR images were independently analyzed by the investigator (JEM) and a radiation oncologist (FC) blinded to patient information and patient outcome. Volumetric measurements of the tumors on the MR images at all three time points were performed by the two readers, independently, and the readers were blinded to each other's results. All measurements were performed on a DICOM based system for diagnostic images, IntelliSpace PACS, (Philips Healthcare, Best, The Netherlands). Using these measurements, a suspected tumor was defined as an isointense mass within the irradiated tumor bed in concordance with the GEC-ESTRO guidelines for depicting gross tumor volume. Tumor volume was calculated by placing freehand regions of interest along the border of the suspected tumor on T2W sagittal or transversal MR images, to obtain the sectional tumor area of each tumor-containing slice, and multiplying the sectional tumor area obtained with section thickness. ${ }^{(18)}$

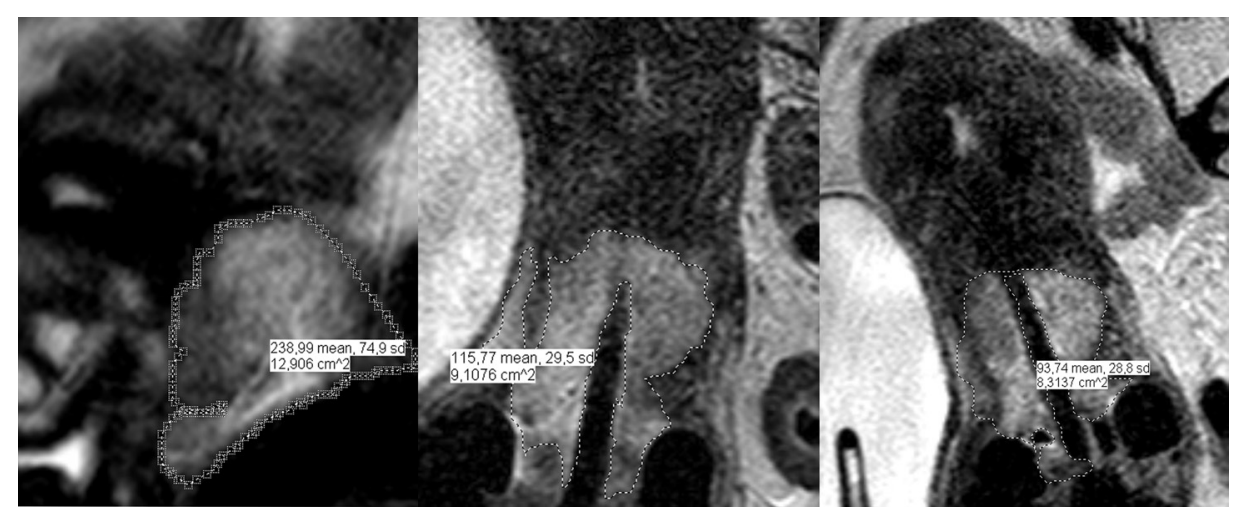

FIGURE 1. Volume measurement of cervical carcinoma, pre-treatment, at first and final BC 
The tumor volume reduction ( $\Delta$ volume) was calculated as the absolute volume obtained from the post-treatment MRI scan minus the initial tumor volume obtained from the pre-treatment MRI scan divided by the initial tumor volume obtained from the pre-treatment MRI scan: ${ }^{(18)}$ ((post-volume - pre-volume) / pre-volume) ${ }^{*} 100$.

\section{STANDARD OF REFERENCE}

The presence or absence of a local residual tumor (in cervix and/or vagina, parametria,

bladder, or rectum) was determined by:

(1) histopathology of the surgical resection specimen $(n=11)$;

(2) the results of a post-treatment gynecologic examination (under anesthesia, with or without biopsy) performed 3 months after completion of the entire radiation treatment and after at least 12 months of documented follow-up ( $n=24)$.

The presence of distant metastases was proven either by histopathology or the detection of a growing tumor mass during subsequent imaging analysis. Overall treatment failure was defined as the presence of local residual or recurrent tumor (histologically proven), distant metastases, or both

\section{STATISTICAL ANALYSIS}

Statistical analyses were performed using SPSS Statistics v18.0 (SPSS Inc, Chicago, Ill.) and Stata v11.0 (StataCorp LP, Texas). Interobserver variations were assessed by means of interclass correlation coefficients for single and average measures.

Receiver operating characteristics (ROC) curves were constructed to evaluate diagnostic performance for (a) absolute MRI volume measurements and (b) volume regression ( $\Delta$ volume). Corresponding areas under the ROC curve $(A \cup C)$, sensitivity, specificity, and positive and negative predictive values were calculated according to the optimal cut off, determined according to the point nearest to the upper left corner in the ROC curve. Sensitivities and specificities for the different scoring methods were compared using the McNemar test for paired data or the chi-squared test for unpaired data. AUCs were compared according to the method described by De Long et al. [19] P-values less than 0.05 were considered statistically significant.

\section{RESULTS}

A total of 48 consecutive patients were identified, of which 35 met the inclusion criteria. Their baseline characteristics are presented in Table 1.

\begin{tabular}{ll} 
TABLE 1. Baseline characteristics & \\
\hline Age median (range) & 53 years (32-77) \\
Histology & $28(28 \%)$ \\
Squamous cell carcinoma & $5(14 \%)$ \\
Adenocarcinoma & $2(6 \%)$ \\
Other & \\
FIGO & $5(14 \%)$ \\
I Ib1 & $4(11 \%)$ \\
II & $5(14 \%)$ \\
IIIb & $17(49 \%)$ \\
III & 0 \\
III & 0 \\
IIIb & $2(6 \%)$ \\
III & $2(6 \%)$ \\
IVa & $29(83 \%)$ \\
Therapy & $6(17 \%)$ \\
Chemo-radiotherapy & \\
Hyperthermia-radiotherapy & \\
\hline FIGO - International Federation of Gynecology and Obstetrics &
\end{tabular}

\section{PATIENT AND TREATMENT CHARACTERISTICS}

After treatment, a local residual tumor was histologically proven by biopsy in four patients, of whom one patient had concurrent distant metastases and one patient had concurrent lymphatic metastases. Six patients had distant metastases only. At a median follow-up of 22 months (range, 13-41), the remaining 25 patients were still free of disease.

\section{MRI VOLUMETRY FOR DETECTION OF LOCAL RESIDUAL TUMOR}

The absolute volume measurements of both observers are shown in Table 2. A significant decrease in tumor volume during treatment was observed in all patients $(p \leq 0.01)$. The AUC, interclass correlation coefficients, sensitivity and specificity for absolute volume, and volume regression are shown in Table 3. There was a good correlation between the absolute volume measurements made by the two observers (interclass correlation coefficients, 0.90-0.98) 
A tumor volume at first $\mathrm{BCT}>11.5 \mathrm{~cm}^{3}$ predicted local residual tumor following treatment $(p<0.03)$. A volume $>9.4 \mathrm{~cm}^{3}$ at the final BCT and $<77 \%$ volume regression compared to the pre-treatment volumes obtained with MRI ( $\triangle$ volume) were associated with a local residual tumor after completion of therapy $(p<0.02)$ (AUC, $0.98-1.00$ ).

\section{MRI VOLUMETRY FOR PREDICTION OF OVERALL TREATMENT FAILURE}

The interclass correlation coefficients, AUC, and the $p$-values for absolute volume and volume regression are shown in Table 4 . A tumor volume $>2.8 \mathrm{~cm}^{3}$ at the final $B C T$ was statistically associated with overall treatment failure $(p<0.03)$ (AUC, 0.79 0.81 ). Also, $<89 \%$ tumor volume regression ( $\Delta$ volume) compared to the pre-treatment volume assessed with MRI scan significantly predicted overall treatment failure (AUC $0.87-0.78)$

TABLE 2. Absolute MRI tumor volume measurements and regression before treatment, at first and at final brachytherapy during radiotherapy treatment of cervical cancer.

\begin{tabular}{lcccc} 
& \multicolumn{3}{c}{ Observer 1 } & \multicolumn{2}{c}{ Oberserver 2 } \\
\cline { 2 - 5 } & volume $\left(\mathrm{cm}^{3}\right)$ & $\Delta$ voluation & volume $\left(\mathrm{cm}^{3}\right)$ & $\Delta$ volume (\%) \\
\hline Local complete response (n:31) & $33(13-104)$ & - & $33(11-130)$ & - \\
(a) MRI before tratment & $8.5(0.4-28)$ & $73 \%(48-97)$ & $8(1-23)$ & $73 \%(37-95)$ \\
(b) MRI at the first BCT* & $3.7(0-14)$ & $88 \%(75-100)$ & $3.3(0.5-9)$ & $89 \%(80-100)$ \\
(c) MRI at the final BCT* & $32(15-44)$ & - & $28(19-37)$ & - \\
Local residual tumor (n:4) & $18(14-23)$ & $36 \%(8-55)$ & $16(10-23)$ & $39 \%(21-68)$ \\
(a) MRI before treatment & $13(4-15)$ & $53 \%(21-67)$ & $11(3-13)$ & $55 \%(28-74)$ \\
(b) MRI at the first BCT* & & & & \\
(c) MRI at the final BCT* & & &
\end{tabular}

(c) MRI at the final BCT*

${ }^{*} \mathrm{p} \leq 0.01$ compared to the residual tumor group

TABLE 3 on page 95

TABLE 4. Diagnostic performance of volume measurements for assessing overall treatment failure using MRI before treatment, at first BCT and final $\mathrm{BC}$.

\begin{tabular}{|c|c|c|c|c|}
\hline \multirow[t]{2}{*}{ Evaluation } & \multicolumn{2}{|c|}{ AUC; Significance } & \multirow{2}{*}{$\begin{array}{l}\text { Interclass coef- } \\
\text { ficient (average) }\end{array}$} & \multirow[t]{2}{*}{ Cut-off } \\
\hline & Observer 1 & Oberserver 2 & & \\
\hline \multicolumn{5}{|l|}{ Absolute volume analysis (n:35) } \\
\hline (a) MRI before treatment & $0.56(0.37-0.75) ;-$ & $0.57(0.38-0.76) ;-$ & $0.98(0.96-0.99)$ & $28,6 \mathrm{~cm}^{3}$ \\
\hline (b) MRI at the first BCT* & $0.82(0.69-0.96) ; p>0.01$ & $0.78(0.63-0.93) ; p=0.02$ & $0.95(0.91-0.98)$ & $7,7 \mathrm{~cm}^{3}$ \\
\hline (c) MRI at the final BCT* & $0.81(0.67-0.96) ; p<0.01$ & $0.79(0.63-0.95) ; p=0.03$ & $0.94(0.89-0.97)$ & $2,8 \mathrm{~cm}^{3}$ \\
\hline \multicolumn{5}{|l|}{ Volume regression analysis $(n: 35)$} \\
\hline First $B C T$ MRI/MRI before treatment & $0.87(0.75-0.99) ; p<0.01$ & $0.78(0.72-1.00) ; p=0.11$ & $0.94(0.87-0.97)$ & $72 \%$ \\
\hline Final BCT MRI/MRI before treatment & $0.84(0.69-0.80) ; p=0.02$ & $0.79(0.63-0.95) ; p=0.02$ & $0.97(0.95-0.99)$ & $89 \%$ \\
\hline
\end{tabular}

AUC are given with the corresponding 95\% confidence intervals.

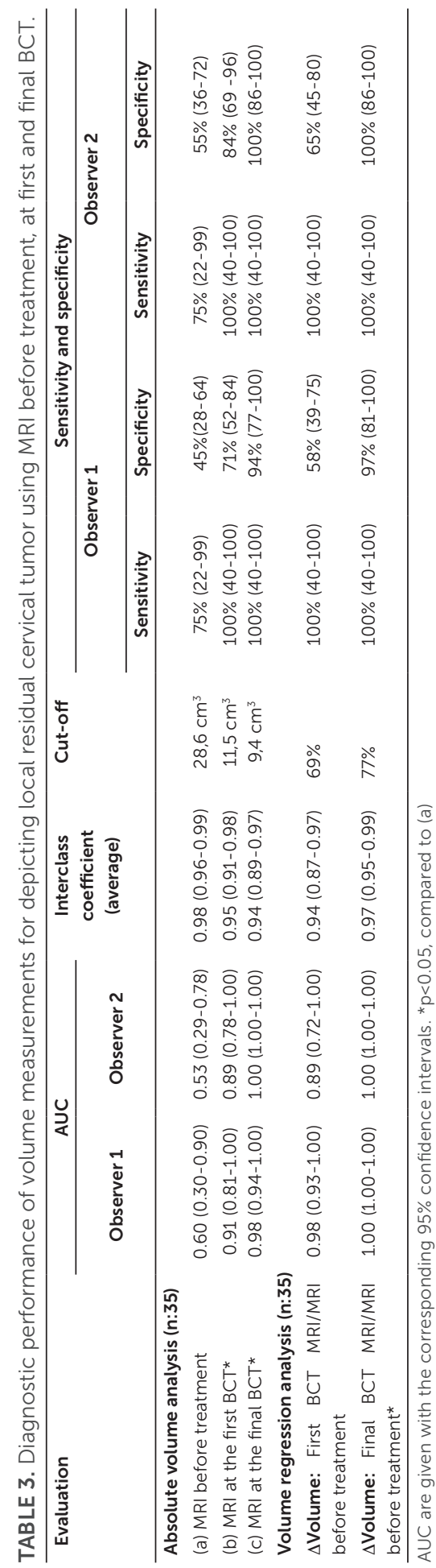




\section{DISCUSSION}

Our study shows that absolute volume measurements as well as volume regression determination at the time of the final $\mathrm{BCT}$ are accurate predictors for treatment outcome. Performing volume measurements, i.e. the absolute tumor volume and tumor volume regression ( $\Delta$ volume) at the time of the final BCT treatment predicted residual tumor after treatment and overall treatment failure during follow-up. Volume analysis at first BCT gave promising results however volumetry during the final BCT was superior for predicting residual disease. Indicative for the impact of BCT treatment for treatment outcome in these tumors. As the results of absolute volume analysis were comparable to those of volume regression analysis, absolute volume measurements for the prediction of residual tumor after BCT could be a better option in general practice because it is less time-consuming.

The presence of persistent disease after radiation treatment is a significant predictor of patient survival, early detection of tumors in this group of patients is of clinical importance. For patients with residual disease situated in cervical or adjacent tissue, surgical resection after radiation treatment can be beneficial. ${ }^{(4,5)}$ To date, most patients receive expectant management until disease recurrence, which might lead to more extensive surgery with increased morbidity or to a situation where the patient might not even be operable because of large tumor size, local involvement of bladder or bowel, distant metastasis, or extensive radiation induced fibrosis. As radiation-induced fibrosis in the target area is lower after completion of EBRT, surgery is an easier option at that time.

Outcome in locally advanced cervix carcinoma has significantly improved through dose escalation enabled by IGABT. ${ }^{(14)}$ The introduction of IGABT resulted in an increase in overall local control (91-95\%) and a reduction of late toxicity by $50-60 \%$. ${ }^{(14)}$ Our results show that volumetric analysis during radiation treatment can predict treatment failure for individual patients. In these patients therapy, could be further tailored to for example surgery and/or adjuvant systemic treatment.

Several groups have performed semi-objective volumetric tumor measurements for the assessment of treatment responses. ${ }^{(10-13,20,21)}$ Most of these studies were performed retrospectively with small study populations, the imaging protocols employed differed from study to study, and volume evaluations were obtained using ellipsoid based volume measurements, which are inferior to those obtained by manual and freehand delineation of regions of interest. 18
Mayr et al. reported the results of MRI-based volume analysis during treatment at a single institution. ${ }^{(10-13)}$ Wang and Mayr et al. prospectively assessed reductions in tumor volumes measured by sequential MRI during treatment and found a correlation between the volume reduction ratio and 5-year local control rates. ${ }^{(13)}$ Volume regression after EBRT (40-50 Gy) and not initial tumor volume was the most accurate predictor for local control and disease free survival. They found that a tumor with an initial volume $>40 \mathrm{~cm}^{3}$ and a residual volume $>20 \%$ after EBRT on an MRI scan is a predictor of local treatment failure (Sensitivity $89 \%$ and Specificity $89 \%$ ). ${ }^{(13)}$

In contrast to our study, Mayr et al. reported performing volumetric analysis without BCT applicators in place, necessitating an additional MRI scan. This consequently increases costs and requires the patient to come in the MR unit at a separate time.

The quality of the MRI performed during brachytherapy with the applicator in situ has shown to be sufficient for brachytherapy treatment planning. System based distortion due to the use of a magnetic field are generally under 1-2 mm and are at their lowest in the center of the image. Additional geometric distortions caused by adding a MRcompatible applicator have shown to be nearly undetectable and are at their lowest in the center of the image. ${ }^{(22-24)}$ In general MRI and applicator induced reconstruction uncertainties are smaller than the uncertainties induced by tumor contouring and bowell movement.

As the use of MRI-guided BCT for a cervical carcinoma larger than $5 \mathrm{~cm}$ is expected to increase significantly the future as more institutions perform 3D treatment planning instead of 2D X-ray, volumetric analyses based on routine MRI could prove to be more cost effective and convenient. ${ }^{(14,16,25,26)}$

The prevalence of recurrent/persistent local tumors in our dataset (11.4\%) is rather low compared to those previously reported. The low recurrence rate could be due to the lower initial tumor volumes, which in $80 \%$ of our population were $<40 \mathrm{~cm}^{3}$, or to several other reasons. First, because of the exclusion criteria, patients who did not finish imaging or the treatment protocol because of early progressive disease were excluded. Second, all patients received together with radiation therapy, concurrent chemotherapy or hyperthermia treatment, which resulted in a higher cure rate than that of radiotherapy alone. ${ }^{(2728)}$ Third, all patients received MRI-based high-dose-rate intracavitary and interstitial BCT according to the GEC-ESTROO guidelines, which is proven to be superior to $2 \mathrm{D}$-based radiotherapy, especially for cervical carcinomas 
sized $>5 \mathrm{~cm} .{ }^{(26)}$ Moreover, we included tumors that were relatively smaller than those reported in other studies

Our study had some limitations. First, the absence of residual disease was not proven histopathologically in all patients. This was mainly because some patients did not undergo surgery. However, we believe that long-term follow-up in the absence of recurrence is at least as good as performing surgico-pathologic analysis. Especially because in those cases the time interval between pathologic analysis with random biopsies or adjuvant surgery is shorter and it could be that because of this reason microscopic tumors are still existent. Possible this would not have been the case with longer waiting time, because of delayed radiotherapy effects. A second limitation of this study was the small number of patients. MRI-based BCT became the standard protocol in our center in October 2008, which was the main reason for the exclusion of 13 patients who underwent only one MRI scan during BCT.

\section{CONCLUSION}

Volume measurement at the time of BCT is a potential promising predictor of local tumor response in individual patients, especially when performed during the final $\mathrm{BCT}$. As absolute volumetric analysis yielded results comparable to those of volume regression analysis, a single tumor volume measurement at the time of the last $B C T$, could already be sufficient to reduce measurement time. We also observed an association between overall treatment failure and local volume response, the potential of assessing local response for identifying individual patients at high risk for overall treatment failure is promising. External validation however in a larger and independent study population is warranted.

\section{COMPETING INTEREST}

This multidisciplinary research (radiology, gynecology and radiation oncology) was performed by GROW (School for Oncology and Developmental Biology, Maastricht, The Netherlands). All authors entitled to authorship are listed as author, there are no other acknowledgments. There are no conflicts of interests to be reported.

\section{REFERENCE}

1. Babar S, Rockall A, Goode A, Shepherd J, Reznek R. Magnetic resonance imaging appearances of recurrent cervical carcinoma. Int J Gynecol Cancer. 2007;17(3):637-45. doi:10.1111/j.1525-1438.2007.00849.x.

2. Elit L, Fyles AW, Devries MC, Oliver TK, Fung-Kee-Fung M. Follow-up for women after treatment for cervical cancer: a systematic review. Gynecol Oncol. 2009;114(3):528-35. doi:10.1016/j.ygyno.2009.06.001.

3. Kitchener HC, Hoskins W, Small W, Jr., Thomas GM, Trimble EL. The development of priority cervical cancer trials: a Gynecologic Cancer InterGroup report. Int J Gynecol Cancer. 2010;20(6):1092-100. doi:10.1111/IGC.0b013e3181e730aa.

4. Hong JH, Tsai CS, Lai CH, Chang TC, Wang CC, Chou HH, et al. Recurrent squamous cell carcinoma of cervix after definitive radiotherapy. Int J Radiat Oncol Biol Phys. 2004;60(1):249-57. doi:10.1016/j. ijrobp.2004.02.044.

5. Houvenaeghel G, Lelievre L, Buttarelli M, Jacquemier J, Carcopino X, Viens P, et al. Contribution of surgery in patients with bulky residual disease after chemoradiation for advanced cervical carcinoma. Eur J Surg Oncol. 2007;33(4):498-503. doi:10.1016/j.ejso.2006.10.011.

6. Nijhuis ER, van der Zee AG, in 't Hout BA, Boomgaard JJ, de Hullu JA, Pras E, et al. Gynecologic examination and cervical biopsies after (chemo) radiation for cervical cancer to identify patients eligible for salvage surgery. Int J Radiat Oncol Biol Phys. 2006:66(3):699-705. doi:10.1016/i.jijobp.2006.05.039.

7. Vincens E, Balleyguier C, Rey A, Uzan C, Zareski E, Gouy S, et al. Accuracy of magnetic resonance imaging in predicting residual disease in patients treated for stage IB2/II cervical carcinoma with chemoradiation therapy : correlation of radiologic findings with surgicopathologic results. Cancer. 2008;113(8):2158-65. doi:10.1002/cncr.23817

8. Hatano K, Sekiya Y, Araki H, Sakai M, Togawa T, Narita Y, et al. Evaluation of the therapeutic effect of radiotherapy on cervical cancer using magnetic resonance imaging. Int J Radiat Oncol Biol Phys. 1999;45(3):639-44.

9. Manfredi R, Maresca G, Smaniotto D, Greggi S, Andrulli D, Rabitti C, et al. Cervical cancer response to neoadjuvant therapy: MR imaging assessment. Radiology. 1998;209(3):819-24.

10. Mayr NA, Magnotta VA, Ehrhardt JC, Wheeler JA, Sorosky JI, Wen BC, et al. Usefulness of tumor volumetry by magnetic resonance imaging in assessing response to radiation therapy in carcinoma of the uterine cervix. Int J Radiat Oncol Biol Phys. 1996;35(5):915-24

11. Mayr NA, Wang JZ, Lo SS, Zhang D, Grecula JC, Lu L, et al. Translating response during therapy into ultimate treatment outcome: a personalized 4-dimensional MRI tumor volumetric regression approach in cervical cancer. Int J Radiat Oncol Biol Phys. 2010;76(3):719-27. doi:10.1016/j.jirobp.2009.02.036

12. Nam H, Park W, Huh SJ, Bae DS, Kim BG, Lee JH, et al. The prognostic significance of tumor volume regression during radiotherapy and concurrent chemoradiotherapy for cervical cancer using MRI. Gynecol Oncol. 2007:107(2):320-5. doi:10.1016/j.ygyno.2007.06.022.

13. Wang JZ, Mayr NA, Zhang D, Li K, Grecula JC, Montebello JF, et al. Sequential magnetic resonance maging of cervical cancer: the predictive value of absolute tumor volume and regression ratio measured 
before, during, and after radiation therapy. Cancer. 2010;116(21):5093-101. doi:10.1002/cncr.25260.

14. Potter R, Haie-Meder C, Van Limbergen E, Barillot I, De Brabandere M, Dimopoulos J, et al. Recommendations from gynaecological (GYN) GEC ESTRO working group (II): concepts and terms in $3 D$ image-based treatment planning in cervix cancer brachytherapy-3D dose volume parameters and aspects of 3D image-based anatomy, radiation physics, radiobiology. Radiother Oncol. 2006;78(1):67-77. doi:10.1016/j.radonc.2005.11.014

15. Harkenrider MM, Alite F, Silva SR, Small W, Jr. Image-Based Brachytherapy for the Treatment of Cervical Cancer. Int J Radiat Oncol Biol Phys. 2015;92(4):921-34. doi:10.1016/j.jirobp.2015.03.010.

16. Potter R, Georg P, Dimopoulos JC, Grimm M, Berger D, Nesvacil N, et al. Clinical outcome of protocol based image (MRI) guided adaptive brachytherapy combined with 3D conformal radiotherapy with or without chemotherapy in patients with locally advanced cervical cancer. Radiotherapy and oncology : journal of the European Society for Therapeutic Radiology and Oncology. 2011;100(1):116-23. doi:10.1016/j.radonc.2011.07.012

17. Beddy P, Rangarajan RD, Sala E. Role of MRI in intracavitary brachytherapy for cervical cancer: what the radiologist needs to know. AJR Am J Roentgenol. 2011;196(3):W341-7. doi:10.2214/AJR.10.5050.

18. Mayr NA, Taoka T, Yuh WT, Denning LM, Zhen WK, Paulino AC, et al. Method and timing of tumor volume measurement for outcome prediction in cervical cancer using magnetic resonance imaging. Int J Radiat Oncol Biol Phys. 2002;52(1):14-22.

19. DeLong ER, DeLong DM, Clarke-Pearson DL. Comparing the areas under two or more correlated receiver operating characteristic curves: a nonparametric approach. Biometrics. 1988;44(3):837-45.

20. Ohara K, Tanaka Y, Tsunoda H, Nishida M, Sugahara S, Itai Y. Assessment of cervical cancer radioresponse by serum squamous cell carcinoma antigen and magnetic resonance imaging. Obstet Gynecol. 2002;100(4):781-7.

21. Saida T, Tanaka YO, Ohara K, Oki A, Sato T, Yoshikawa H, et al. Can MRI predict local control rate of uterine cervical cancer immediately after radiation therapy? Magn Reson Med Sci. 2010;9(3):141-8.

22. Aubry JF, Cheung J, Morin O, Beaulieu L, Hsu IC, Pouliot J. Investigation of geometric distortions on magnetic resonance and cone beam computed tomography images used for planning and verification of high-dose rate brachytherapy cervical cancer treatment. Brachytherapy. 2010;9(3):266-73. doi:10.1016/j. brachy.2009.09.004.

23. Hellebust TP, Kirisits C, Berger D, Perez-Calatayud J, De Brabandere M, De Leeuw A, et al. Recommendations from Gynaecological (GYN) GEC-ESTRO Working Group: considerations and pitfalls in commissioning and applicator reconstruction in 3D image-based treatment planning of cervix cancer brachytherapy. Radiotherapy and oncology : journal of the European Society for Therapeutic Radiology and Oncology. 2010;96(2):153-60. doi:10.1016/j.radonc.2010.06.004.

24. Zhou J, Zamdborg L, Sebastian E. Review of advanced catheter technologies in radiation oncology brachytherapy procedures. Cancer management and research. 2015;7:199-211. doi:10.2147/CMAR. S46042.

25. Dimopoulos JC, Petrow P, Tanderup K, Petric P, Berger D, Kirisits C, et al. Recommendations from
Gynaecological (GYN) GEC-ESTRO Working Group (IV): Basic principles and parameters for MR imaging within the frame of image based adaptive cervix cancer brachytherapy. Radiother Oncol. 2012. doi:10.1016/j.radonc.2011.12.024

26. Potter R, Dimopoulos J, Georg P, Lang S, Waldhausl C, Wachter-Gerstner N, et al. Clinical impact of MRI assisted dose volume adaptation and dose escalation in brachytherapy of locally advanced cervix cancer. Radiother Oncol. 2007:83(2):148-55. doi:10.1016/j.radonc.2007.04.012.

27. (CCCMAC). CfCCM-aC. Reducing uncertainties about the effects of chemoradiotherapy for cervical cancer: individual patient data meta-analysis. Cochrane Database Syst Rev. 2010;Jan 20;(1).

28. Lutgens L, van der Zee J, Pijls-Johannesma M, De Haas-Kock DF, Buijsen J, Mastrigt GA, et al. Combined use of hyperthermia and radiation therapy for treating locally advanced cervix carcinoma. Cochrane Database Syst Rev 2010(3) 


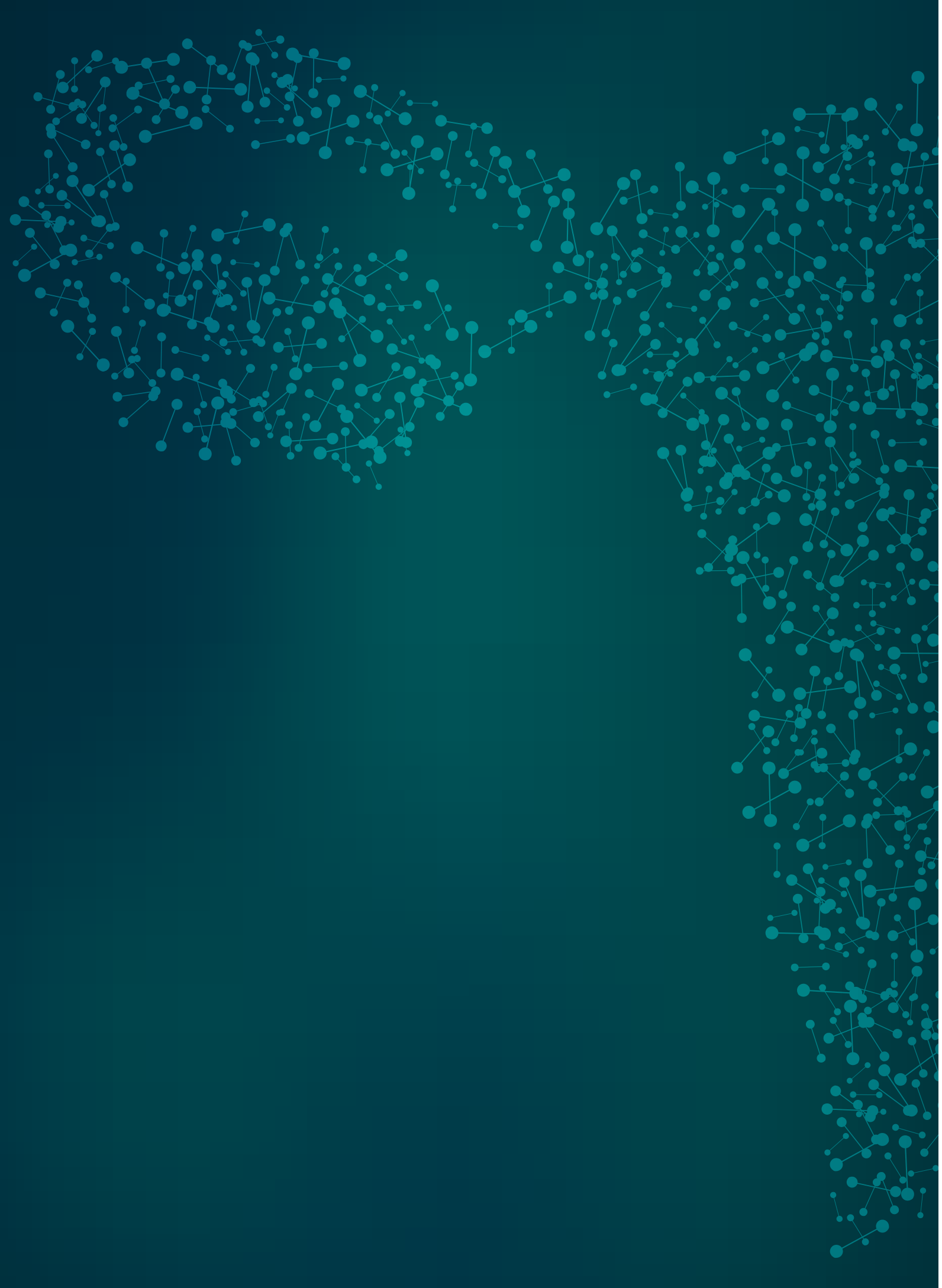

${ }^{18}$ F-FDG POSITRON EMISSION TOMOGRAPHY MAGNETIC RESONANCE IMAGING (PET-MRI) FOR RESPONSE ASSESSMENT AFTER CHEMO-RADIATION THERAPY OF CERVICAL CARCINOMA: AN INITIAL EXPERIENCE.

\footnotetext{
Mongula JE Bakers FCH

de Voo S

Lutgens L

van Gorp T

Kruitwagen RFPM

Slangen BFM
} 


\section{ABSTRACT}

\section{PURPOSE}

Advanced stage cervical cancer is primarily treated by radiotherapy. Local tumor control is a prerequisite for cure. Imaging after treatment is controversial. Positron Emission Tomography (PET) combined with computer tomography (PET-CT) shows great promise for detecting metastases. On the other hand, Magnetic Resonance Imaging (MRI) is superior in depicting anatomical details. The combination of PET-MRI could result in more accurate evaluation of cervical cancer treatment outcome. The aim of this pilot study is to share our initial experience with PET-MRI in the evaluation of treatment response in cervical cancer after radiation treatment.

\section{METHODS}

Ten patients with cervical carcinoma (FIGO >IB2) were prospectively evaluated. Eleven weeks (median; range 8-15 weeks) after radiation therapy, treatment response was evaluated by PET-MRI. The PET, MRI and combined PET-MRI images were evaluated for the presence of local residual tumor and metastasis. Diagnostic performance was assessed by area under the reciever operator characteristics (ROC) curve for evaluation of local residual tumor. The readers were blinded for outcome data. Local residual disease, metastasis, diagnostic confidence and change of opinion were scored on a 5-point Likert scale. The reference standard consisted of pathology and/or follow-up according to the clinical guidelines.

\section{RESULTS}

Three out of ten patients had local residual abnormalities suggestive for tumor residue after radiation treatment. The availability of both PET and MRI resulted in an increase in diagnostic confidence in $80-90 \%$ of all patients. Change of opinion was observed in $70 \%$ and change in policy in 50\%, especially in the group with residual tumor. The diagnostic accuracy increased significantly for the radiologist if PET-MRI was combined (AUC .54 versus . 83).

\section{CONCLUSION}

PET-MRI shows promise for evaluation of treatment response after radiation for cervical cancer, especially increasing diagnostic confidence, while potentially increasing diagnostic performance.

\section{INTRODUCTION}

Radiation treatment for cervical cancer is recommended for advanced disease (International Federation of Gynecology and Obstetrics (FIGO) stages $\geq 1 \mathrm{lb} 2$ ). In advanced disease, one third of the patients will develop recurrent or progressive disease, the majority occurring in the first two years. ${ }^{(1)}$ Local persistent tumor is responsible for one fourth of treatment failure; lymph node metastasis and distant metastasis are responsible for the remaining part. (2)

For the first group of patients (local persistent tumor), potential therapeutic options depend on the extent of the local tumor and/ or the presence of metastasis. ${ }^{(1)}$

To date, routine surveillance imaging after radiation treatment is not used in daily practice, partly because of poor performance of diagnostic tests. ${ }^{(3)}$ Second, the timing of the follow-up evaluation remains unclear as tumor regression rate varies. Early assessment could lead to an overestimation of redisual tumor, on the contrary late assessment could miss the opportunity to treat asymptomatic persistent disease. ${ }^{4}$

Fluordeoxyglucose-positron emission tomography (FDG-PET) has high potential for predicting response and metastasis after treatment.(5-7) Siva et al. evaluated therapy response by PET-CT three months after treatment. ${ }^{(7)}$ Besides excellent survival for the complete response group, they also showed a $78 \%$ overall survival after salvage therapy for patients with isolated local and nodal residual disease.

In general, FDG-PET is combined with low-dose computed tomography (CT) images. The morphologic resolutions of these CT images are rather poor as compared to MRI. As a consequence, if local treatment failure is suspected based on PET-CT additional Magnetic Resonance Imaging (MRI) is necessary for adequate treatment planning. ${ }^{\left({ }^{(8)}\right.}$ However, the detection of persistent tumors by MRI is complicated by the occurrence of false positive results, mainly due to post-radiation induced fibrosis, inflammation induced edema and necrosis. MRI criteria sets and diffusion weighted MRI imaging could decrease false positive results to some extent but a further increase in diagnostic performance is warranted. ${ }^{(9,10)}$ The combination of the superior morphologic MRI with functional FDG-PET imaging could result in a more accurate diagnosis, staging and follow-up of cervical cancer. ${ }^{(11,12)}$ The functional imaging of the FDG-PET could reduce false positive results of MRI by allowing for differentiation between residual tumor and radiation induced fibrotic tissue. FDG uptake is not expected in fibrotic tissue in comparison to residual tumor. PET is able to assess (lymph node) metastasis, potentially 
resulting in a better treatment allocation after radiation treatment compared to MRI alone. ${ }^{(5,7)}$

The aim of our study was to share our initial experience with the PET-MRI in the evaluation of radiotherapy treatment to discriminate between patients with a complete local response and patients with local residual tumor. We evaluate the potential value of combined PET-MRI in comparison to the MRI and PET component.

\section{METHODS AND MATERIALS}

\section{PATIENTS}

This is a prospective cohort study of 11 consecutive women with histologically proven advanced stage primary cervical cancer (FIGO stages $\geq$ Ib2-IVB), which were referred to Maastricht University Medical Center between July 2014 and October 2015. All patients were discussed in a multi-disciplinary team consisting of gynecologic oncologist, medical oncologist, radiation oncologist, pathologist, radiologist, and a nuclear physician. Patients allocated to radiotherapy (FIGO stages $\geq \mid \mathrm{b} 2$, according to the clinical guidelines) were included.

Ethical approval was given and informed consent for the use of (coded) images was waived by the Maastricht University Medical Centre ethical committee, as the data was analyzed anonymously in accordance with the Institutional Review Board guidelines (IRB no. 13-02-2015)

Radiotherapy was performed according to the current GEC-ESTRO guidelines; this consisted of External Beam Radiation Therapy (EBRT) followed by MRI-guided brachytherapy (BCT). The first BCT was applied during the fifth week of radiotherapy, and the overall treatment time for radiotherapy was $6-7$ weeks. Patients were treated with either concurrent chemotherapy or hyperthermia (if concurrent chemotherapy was contraindicated or in case of FIGO IVB disease after neoadjuvant chemotherapy) according to local clinical guidelines.

\section{PET/MRI IMAGING PROTOCOL}

A whole-body [18F]-FDG-PET/MRI was performed on a 3.0-Tesla Biograph mMR PET/ MRI scanner (Siemens) with a 4.4-mm PET resolution (full width at half maximum). Patients were fasting for at least 6 hours before the examination, a blood glucose level (all $<10 \mathrm{mmol} / \mathrm{L}$ ) was obtained in all patients. Body weight adapted intravenous administration of $2 \mathrm{Mbq} / \mathrm{kg}$ of 18 fluorodeoxyglucose was performed 45 minutes before the PET-MRI. The PET images were acquired in 5-min bed positions. Attenuation correction was performed with a dual-echo VIBE DIXON that separates water and fat with TE1/TE2 $=1.23 \mathrm{msec} / 2.46 \mathrm{msec}, \mathrm{TR}=3.6 \mathrm{msec}$, left-right FOV $=500 \mathrm{~mm}$ and anterior-posterior FOV $=300 \mathrm{~mm}$. The PET acquisition was reconstructed using the Siemens HD reconstruction algorithm ( 3 iterations, 21 subsets, 4 mm Gauss, matrix size 300) and corrected for attenuation, scatter, randoms, dead-time, radioactive decay. PET/MRI images were fused and analyzed using the dedicated DICOM software (Osirix MD, Geneva). 
Quantification of FDG uptake was performed by assessing the standardized uptake value (SUV; measured activity concentration [Bq/ml] xbody weight [g]/injected activity [Bq]).

A total body (from skull-base to groin) 2D T2-weighted fast spin-echo image in two planes (sagittal and axial) was performed (TR 2150/ TE 138 msec, 33 ETL, 1NSA, $(0.98 \times 0.98) \times 6.50 \mathrm{~mm} 3)$

Second, diagnostic pelvic MRI images were performed in three planes (sagittal, axial, and coronal), with the axial and coronal planes angled perpendicular and parallel to the cervical axis, respectively. (2D T2-weighted (T2W) fast spin-echo images (TR 4000/ TE $102 \mathrm{msec}, 25 \mathrm{ETL}, 3 \mathrm{NSA},(0,98 \times 0,98) \times 3.00 \mathrm{~mm} 3$ voxel at 3.0T). Further more the protocol consisted of a T1 in the coronal plane (TR 700/ TE $11 \mathrm{msec}, 57 \mathrm{ETL}, 1 \mathrm{NSA}$, $(0,98 \times 0,98) \times 1,1 \mathrm{~mm} 3$ voxel at 3.0T) and diffusion weighted images (TR 9000/ TE 80 msec, 1 ETL, 3 NSA, $(0,98 \times 0,98) \times 5.0 \mathrm{~mm} 3$ voxel at 3.0T) with B-values $(50,400$ and 800$)$.

Patients did not receive bowel preparation, bladder catheterization or anti-spasmodic agents

\section{ADITIONAL SCANS}

The pre-treatment MRI was performed according to the same protocol on the 3.0 Tesla Bioraph mMR PET-MR (Siemens Magnetom Avanto, Erlangen, Germany) or on a 1.5-Tesla MRI unit (Intera Achieva); Philips Healthcare, Best, The Netherlands (in 5 patients). The protocol used in the latter was a standard protocol for diagnostic pelvic MRI images comparable with the 3.0 Tesla PET-MR protocol. Consisting of T2W, T1W and diffusion weighted images.

PET pre-treatment was performed according to the same protocol on the 3.0 Tesla PET-MR or in 5 patients on a PET-CT scanner Gemini TF TOF 64 (Philips Healthcare, Best, The Netherlands) according to a similar protocol as described for the PET-MR.

\section{IMAGE EVALUATION}

In The Netherlands, radiologic assessment is done by a radiologist and assessment of nuclear imaging by a nuclear physician. If combined imaging, like PET and CT, is used, the radiologist and nuclear physician evaluate their findings together and give their combined result to the clinician. The MRI images were retrospectively independently analysed by a radiologist (FB) and the PET images by a nuclear physician $(\mathrm{SdV})$ with experience in oncologic imaging blinded to all patient information and patient outcome. After the initial analysis both readers evaluated the PET-MRI images together, they discussed their separate findings and came to consensus. ROC curves to determine area under the curve were performed.

The readers were asked to assess the presence of residual tumor and/or metastasis based on a 'subjective' visual assessment of the images using the following confidence level scores: 0 , definitely no residual tumor/metastasis; 1 , probably no residual tumor/ metastasis; 2, unclear; 3, probably residual tumor/metastasis; 4, definitely residual tumor/metastasis. The readers were not given any instructions or asked to search for certain criteria, and were free to interprete the scans based on prior experience.

Second, at the consensus meeting, they scored diagnostic confidence. This was scored on a similar 5 -point Likert scale: 0 , certainly no increase in confidence; 1 , probably no increase; 2 , unclear; 3 , probably increase; 4 , definitely increase. Change in diagnostic confidence was calculated with a predefined cut-off (score 2); only score 3 and 4 were seen as a change of diagnostic confidence.

All measurements were performed on a dedicated DICOM system (Osirix MD, Geneva).

\section{STANDARD OF REFERENCE}

Local residual tumor was defined as tumor at the original tumor site (cervix, vagina, parametria, bladder, or rectum). The presence or absence of a local residual tumor was determined by:

(1) histopathology of the surgical resection specimen

(2) the results of a post-treatment gynecologic examination (under anesthesia, with or without biopsy) performed 3 months after completion of the entire radiation treatment and at least 12 months of documented follow-up. Follow-up consisted of 3 monthly check-up by alternating a gynecologic and radiation oncologist and if indicated with additional imaging and/or tumor markers conform clinical guidelines.

Regional residual tumor was defined as residual and/or recurrent lymphatic tumor within the irradiated volume (i.e. pelvic or lower para-aortic lymphnodes). Distant metastasis was defined as metastasis outside of the irradiated volume. Regional tumor and distant metastasis were proven either by histopathology or the detection of a growing tumor mass during subsequent imaging analysis.

After radiation therapy in case of isolated local residual disease without metastasis, patients were subjected to salvation surgery. If distant metastasis was present; palliative 
therapy was proposed. In case of no evidence of disease, follow-up was performed according to the clinical guidelines.

\section{STATISTICAL ANALYSIS}

Statistical analyses were performed using SPSS Statistics v20.0 (SPSS Inc, Chicago, Ill.) and Stata v11.0 (StataCorp LP, Texas).

Receiver operating characteristics $(\mathrm{ROC})$ curves were constructed to evaluate diagnostic performance for (a) MRI, (b) PET and (c) combined PET-MR analysis. Corresponding areas under the ROC curve (AUC) were compared according to the method described by De Long et al. ${ }^{(13)}$ P-values less than 0.05 were considered statistically significant.

\section{RESULTS}

\section{PATIENT AND TREATMENT CHARACTERISTICS}

Between July 2014 and October 2015, out of a prospective cohort of 11 patients, 10 patients obtained a PET-MRI. One patient did not have PET/MRI due to technical problems. Table 1 shows the baseline characteristics.

\begin{tabular}{|c|c|c|c|c|c|c|}
\hline Patient & $\begin{array}{l}\text { Age } \\
\text { year }\end{array}$ & $\begin{array}{l}\text { Histological } \\
\text { sub-type }\end{array}$ & FIGO & $\begin{array}{l}\text { Diameter } \\
\mathrm{Cm}\end{array}$ & $\begin{array}{l}\text { Para -iliacal Lymph nodes } \\
\text { suspected on MR or PET-CT }\end{array}$ & Therapy \\
\hline 1 & 42 & Squamous cell & $\| B$ & 5 & + & CRT \\
\hline 2 & 51 & Squamous cell & $\| B$ & 6 & - & CRT \\
\hline 3 & 65 & Adenosquamous & $\| B$ & 7 & + & CRT \\
\hline 4 & 60 & Squamous cell & $\| B$ & 4 & & CRT \\
\hline 5 & 45 & Squamous cell & $\mathrm{IB}$ & 5 & & CRT \\
\hline 6 & 41 & Squamous cell & $\| B^{*}$ & & + & Neoadjuvant $\mathrm{CHT}+\mathrm{HT}$ \\
\hline 7 & 50 & Adenocarcinoma & $\| B^{* *}$ & 6 & + & $\begin{array}{l}\text { Neoadjuvant } \mathrm{CHT}+\mathrm{HT}+\mathrm{RT} \\
\text { on oligometastase }\end{array}$ \\
\hline 8 & 30 & Adenosquamous & $\| B$ & 5 & & CRT \\
\hline 9 & 53 & Squamous cell & IVA & 4 & - & Neoadjuvant $\mathrm{CHT}+\mathrm{HT}$ \\
\hline 10 & 27 & Adenocarcinoma & IB2 & 4 & + & CRT \\
\hline
\end{tabular}

All patients recieved treatment with curative intent. Two patients (patient 6 and 7) had distant metastasis (jugular lymph node and glenoid) on pretreatment MR/PET-CT, they recieved neoadjuvant chemotherapy (clinical stage IIB, histological stage IVB). The median time interval between last treatment and the PET/MRI was 11 weeks (8-15 weeks)

\section{DIAGNOSTIC CONFIDENCE, CHANGE OF OPINION AND CHANGE POLICY}

Table 2 shows the score for residual disease based on MRI, PET and PET-MRI consensus.

The reference standard, outcome and additional treatments are shown. The functional MRI and PET parameters (ADCmean and SUVmax) after treatment are shown.

Table $\mathbf{3}$ and $\mathbf{4}$ show the score for residual and distant disease together with the change of opinion and change of policy for both the MRI alone, PET alone and both modalities combined.

Table 5 shows the diagnostic accuracy and diagnostic confidence. 
TABLE 2. Score for residual tumor based on MRI (Radiologist), PET (Nuclear Physician) and PET-MRI (consensus Radiologist and Nuclear Physician) performed 3 months after treatment, quantitative measurements, reference standard and outcome.

\begin{tabular}{|c|c|c|c|c|c|c|c|c|}
\hline \multirow[b]{2}{*}{ Patient } & \multirow{2}{*}{$\begin{array}{l}\mathrm{MRI}_{\text {post }} \\
\mathrm{ADC}_{\text {mean }}\end{array}$} & \multirow{2}{*}{$\begin{array}{l}\mathrm{PET}_{\text {post }} \\
\mathrm{SUV}_{\text {max }}\end{array}$} & \multirow{2}{*}{$\begin{array}{c}\text { MRI } \\
\text { Residual }\end{array}$} & \multirow{2}{*}{$\begin{array}{c}\text { PET } \\
\text { Residual }\end{array}$} & \multirow{2}{*}{$\begin{array}{l}\text { MRI-PET } \\
\text { Residual }\end{array}$} & \multicolumn{2}{|c|}{ Reference-standard } & \multirow[b]{2}{*}{ Outcome } \\
\hline & & & & & & $\begin{array}{c}\text { Local } \\
\text { disease }\end{array}$ & $\begin{array}{c}\text { Distant } \\
\text { Metastasis }\end{array}$ & \\
\hline 1 & 1.5 & 1.8 & - & - & $\cdots$ & No & No & FU: NED 20 months \\
\hline 2 & 1.4 & 2.8 & & & - - & No & No & FU: NED 26 months \\
\hline 3 & 1.3 & 2.9 & & $+1-$ & - - & No & No & FU: NED 25 months \\
\hline 4 & 1.3 & 3.0 & + & + & $+/-$ & No & No & FU: NED 27 months \\
\hline 5 & 0.7 & 3.2 & & + & & Yes* & Yes & Palliation CHT \\
\hline 6 & 1.3 & 3.6 & + & ++ & ++ & Yes & Yes & Palliation $\mathrm{CHT}$, diseased \\
\hline 7 & 1.6 & 3.0 & + & & $+/-$ & No & Yes & Multiple meta, palliative \\
\hline 8 & 2.0 & 2.6 & $+/-$ & & + & No & No & $\begin{array}{l}\text { Salvage surgery, PA: no } \\
\text { tumor. NED: } 21 \text { months }\end{array}$ \\
\hline 9 & 1.5 & 5.1 & $+/-$ & ++ & + & Yes & No & $\begin{array}{l}\text { Salvage surgery, FU: Liver } \\
\text { metastasis } 3 \text { months after } \\
\text { surgery. } \\
\text { Palliative CHT }\end{array}$ \\
\hline 10 & 1.6 & 7.3 & - & + & - - & No & Yes & Palliative $\mathrm{CHT}$ \\
\hline
\end{tabular}

Corresponding symbols: - - , definitely no residual tumor; - , probably no residual tumor; +/-, unclear possibly residual tumor; + , probably residual tumor; ++ , definitely residual tumor/metastasis. Abbreviation: PA: pathology, NED: no evidence of disease, FU: Follow-up, CHT: chemotherapy. *No Pathology of local residual disease, growing mass on subsequent scans.

TABLE 3. Local residual tumor based on MRI (Radiologist), PET (Nuclear Physician) and PET-MRI (consensus Radiologist and Nuclear Physician) scores, change of opnion and change of policy.
TABLE 4. Metastases based on MRI (Radiologist), PET (Nuclear Physician), PET-MRI (consensus Radiologist and Nuclear Physician) and the reference standard, change of opinion and policy.

\begin{tabular}{cccclll}
\hline \multirow{2}{*}{ Patient } & $\begin{array}{c}\text { MRI } \\
\text { Metastases }\end{array}$ & $\begin{array}{c}\text { PET } \\
\text { Metastases }\end{array}$ & $\begin{array}{c}\text { MRI-PET } \\
\text { Metastases }\end{array}$ & $\begin{array}{l}\text { Regional or } \\
\text { distant disease }\end{array}$ & $\begin{array}{l}\text { Change of opinion } \\
\text { (reason) }\end{array}$ & Policy change \\
\hline 1 & TN & TN & TN & - & - & - \\
2 & TN & TN & TN & - & - & - \\
3 & TN & TN & TN & - & - & - \\
4 & TN & Equivocal & Equivocal & $\begin{array}{l}\text { t primair lung } \\
\text { tumor }\end{array}$ & $\begin{array}{l}\text { t second primair lung } \\
\text { tumor }\end{array}$ & $\begin{array}{l}\text { + additional } \\
\text { therapy }\end{array}$ \\
5 & TP & TP & TP & + pelvic node & - & - \\
6 & FN & TP & TP & + multiple & + FDG positive me- & + palliation \\
7 & FN & FN & FN & + multiple & - fases negative & - \\
8 & TN & TN & TN & - & - & - \\
9 & TN & TN & TN & - & - & - \\
10 & FN & TP & TP & + para-aortic & + FDG positive me- & + palliation \\
& & & & node & tastases & \\
\hline
\end{tabular}

Abbreviation: TN: True negative, TP: True positive, FP: False positive, FN: False negative +: positive, -negative,

TABLE 5. Diagnostic performance, confidence, change of opinion for MRI, PET and PET-MRI for

\begin{tabular}{|c|c|c|c|c|}
\hline assessing & local & residual & cervical & cancer \\
\hline Modality & AUC (Std. Err.) & p value & Diagnostic confidence & Change of opinion \\
\hline $\begin{array}{l}\mathrm{MRI} \\
\end{array}$ & $0.55(0.24)$ & 0.008 & $80 \%$ & $30 \%$ \\
\hline PET & $0.95(0.06)$ & 0.65 & $90 \%$ & $30 \%$ \\
\hline PET-MRI & $0.83(0.16)$ & & & - \\
\hline
\end{tabular}

After radiation treatment, one patient had local residual tumor only; two patients had distant metastasis only and two had both local residual disease and distant metastasis. The remaining four patients had no evidence of disease after a median follow-up of 25 months (range 20-27 months) (Table 2). Two patients with suspected isolated local residual tumor underwent salvage surgery. In one patient (patient 8 ) salvage surgery was performed based on subsequent PET-MRI imaging and 2 biopsies showing possible minimal residual tumor. Pathologic examination of the uterus after salvage surgery however did not show active residual tumor, therefore this patients was regarded as negative for local residual cancer. During the PET-MRI consensus meeting the radiologist had a suspicion for local residual disease based on an isointense, nodular lesion in the cervix. The nuclear physician did not see any FDG hotspots, however the scan was hard to interpreted due to low FDG uptake. Based on the MRI signs they came to consensus and false positively decided there probably was residual tumor. 
For MRI the accuracy improved mainly due to increase in confidence of the observer. Patient 9 was scored doubtful on initial MRI, however the availability of PET resulted in a correct change of opinion to probably local residual tumor. Also 2 MRI's were scored as probably residual disease however after the consensus meeting these patients were downgraded to uncertain, therefore diminishing false positive results. Due to the small population both shifts in scores result in a relatively large increase in AUC.

Patient 5 had a false negative score for residual local disease both on MRI and PETMRI, but because of the presence of distant metastasis, this result had no therapeutic consequences. However, if metastases would not have been present the PET result (probably residual disease) would have had clinical consequences. The local residual disease was shown by an increasing local mass with subsequent imaging

The two patients (patient 6 and 10) with local residual disease and distant metastasis were correctly depicted by the PET component, MRI did not show these metastases.

Second, table $\mathbf{3}$ and $\mathbf{4}$ shows the cases were a change of policy occurred due to the consensus meeting. A change of policy was shown, mainly due to the decrease of falsenegative results. For example patient 9 based on MRI, would have been allocated to followup with additional imaging, became candidate for salvage surgery based on PET-MRI.

For patients 6 and 7, based on MRI, an isolated local disease was suspected, combination with PET revealed the presence of distant metastasis so that salvage surgery was omitted. On the contrary, patient 8 was incorrectly classificated as residual tumor by combined PET-MR analyses, but pathology of the biopsies were inconclusive as well. For patient 4, PET and PET-MR imaging showed a second primair tumor, resulting in additional treatment for this patient.

\section{DIAGNOSTIC PERFORMANCE FOR ASSESSMENT OF RESPONSE}

Figure 1 shows the T2W MRI, DW-MRI corresponding ADC map, FDG-PET and the fusion images in a patient with local residual tumor after chemo-radiation treatment. Additional Figure 2 shows PET-MR images showing para-aortal lymph node metastasis. Table 5 shows the diagnostic performance of both readers based on their individual 'subjective' visual response and there consensus 'subjective' response.

Despite our small number of patients PET-MRI evaluation showed a statistical higher AUC 0.83 versus 0,54 for MRI alone $(p<0,01)$. Compared to PET analyses no statistical difference could be shown

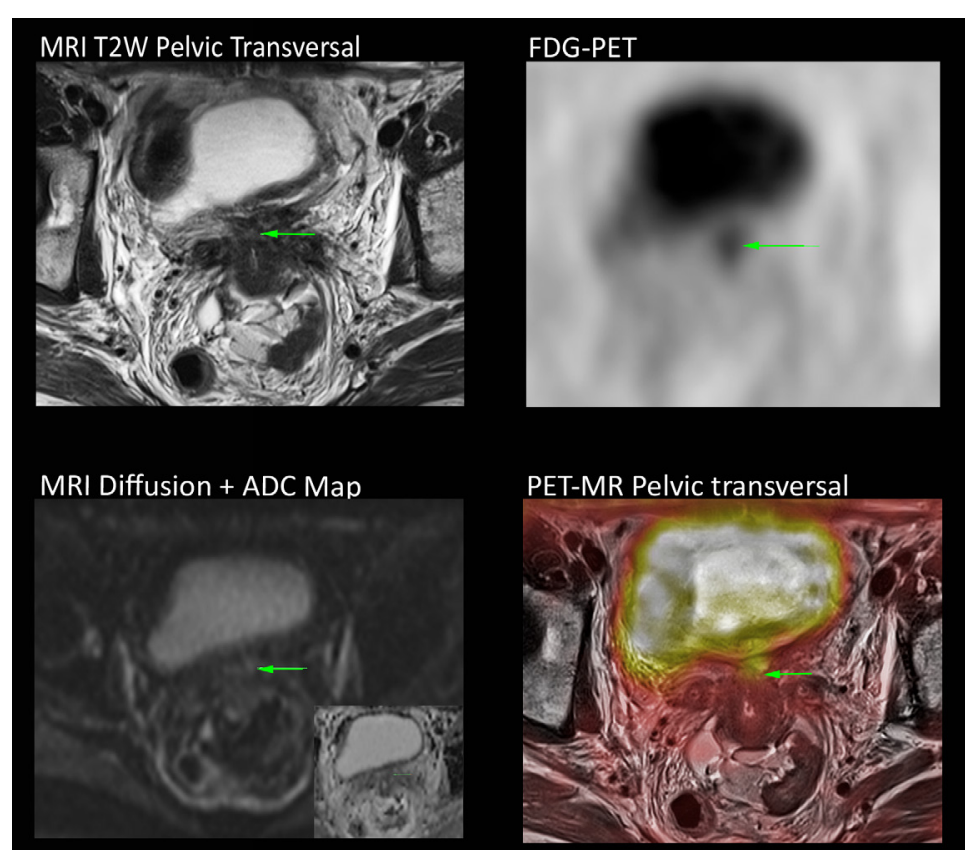

FIGURE 1. MRI T2W, diffusion weighted MRI with ADC map, FDG-PET, PET-MRI fusion showing local residual cancer (green arrow).

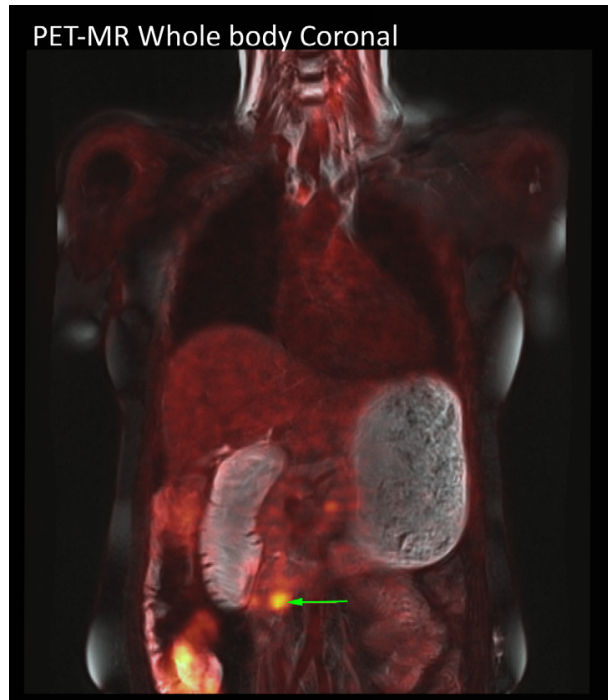

FIGURE 2. Whole-body PET-MRI fusion showing para-aortal lymph node metastasis (green arrow) 


\section{DISCUSSION}

In this pilot study we showed an increase in diagnostic confidence for both the Radiologist and Nuclear Physician. Our results show that this increase in confidence is accompanied by an increase in diagnostic accuracy for assessing local residual cervical carcinoma after radiation treatment.

This is of major importance for the clinical setting, to make correct treatment decisions by the multi-discplinary team. The combined assessment of the PET-MRI resulted in a change of policy in 50 percent of patients.

Our hypothesis that the differentiation between active residual tumor and fibrotic tissue could be diminished by the additional PET data was partly correct as in 2 of 10 patients false positive MRI results could be corrected. We also showed a correct upstaging to probably residual disease with the PET data available, decreasing false negative results.

This is the first study assessing PET-MRI for evaluation of response 3 months after radiation treatment. Multiple research groups showed surveillance PET-CT 3 month after treatment to be predictive for tumor response, moreover Siva et al. allocated patients with isolated local and nodal disease to salvage treatment with a high overall survival rate. ${ }^{(5,7)}$

MRI is also proposed as a surveillance scan 3 months after radiation treatment. However response assessment is hampered by radiation induced changes as fibrosis, necrosis, inflammation and edema, false-positive results up to $50 \%$ are common. ${ }^{9}$, 10) These findings are supported by a recent study by Kim et al., they showed $60 \%$ of patients with residual cervical cancer detected on post-radiation treatment MRI to remain disease free without disease progression. ${ }^{(4)}$ Careful observation without salvage surgery was suggested as an option for patients with tumor $<2 \mathrm{~cm}$ on posttreatment MRI. These findings highlight the necessity for more accurate evaluation of MRI to diminish false-positive results and to predict which suspected MRI lesions will progress over time.

Future studies will give more insight on the value of MRI and the timing of tumor regression after radiation treatment. Additional information about tumor biology could also diminish false positive results by differentiating between active residual cervical cancer and radiation induced changes/non-active residual cancer in regression.
Diffusion-weighted MRI showed some potential based on Apparent Diffusion Coefficient (ADC), cut-off values remain unclear. ${ }^{(14)}$ However, especially tumor glucose uptake as assed by PET could prove to be of value, however no studies perfomed both MRI and PET after radiation treatment to assess residual cervical cancer. Possible our false-positive PET-MR result in one patient could be explained by a delayed tumor regression after radiation treatment.

Due to our small study population we were not able to assess the quantitative functional data of the PET and MRI component. These Apparent Diffusion Coefficient (ADC) and Standard Uptake Value (SUV) are promising functional parameters in the course of radiation treatment. Due to the hybrid combination of PET and MRI a reliable comparison between these 2 parameters becomes available.

In future trials we will assess these parameters as these might be independent and subsequently both potentially increase diagnostic accuracy. ${ }^{(15,16,17}$

PET-MR has previously been used for suspected recurrence of gynecological malignancies including cervical carcinoma. A study performed by Gruneisen et al. showed PET-MR to be superior to whole-body MRI for detecting suspected recurrence while improving diagnostic confidence for the observers. This result is in concordance with our recent findings. ${ }^{(11)}$

The same study group also evaluated PET/MRI versus PET/CT for suspected recurrence of gynecological malignancies. They showed comparable diagnostic accuracy for both methods. For diagnostic confidence of the readers PET/MRI showed higher values for both malignant and benign lesions. Due to the small study populations with different gynecologic malignancies and different histological subtypes these results have to be interpreted with caution. ${ }^{(11,12)}$

Our study has some limitations. A main limitation is our small patient population; therefore conclusions have to be interpreted with caution. Despite our small population promising results supporting the use of PET-MRI for evaluation of cervical carcinoma after radiation treatment could be shown. All patients allocated to radiation treatment by our team were consecutively scanned with the exception of one patient (no PETMRI performed); therefore the risk of selection bias is rather low.

Second, due to the nature of treatment and follow-up it is not possible to have pathology as the golden standard in patients with a complete response. But we believe 
that we were able to provide sufficient follow-up, making false negative results rather unlikely.

In conclusion, a combined PET-MR evaluation shows promising results for assessing treatment response after radiation treatment for cervical cancer, especially increasing diagnostic confidence of the observers. This report shows the benefit of PET addition to MRI as both false positive and false negative MRI findings could be diminished by the addition of the PET.

\section{REFERENCE}

1. Sardain $\mathrm{H}$, Lavoue V, Redpath $\mathrm{M}$, Bertheuil $\mathrm{N}$, Foucher $\mathrm{F}$, Leveque J. Curative pelvic exenteration for recurrent cervical carcinoma in the era of concurrent chemotherapy and radiation therapy. A systematic review. Eur J Surg Oncol. 2015:41(8):975-85

2. Kim TH, Kim MH, Kim BJ, Park SI, Ryu SY, Cho CK. Prognostic Importance of the Site of Recurrence in Patients With Metastatic Recurrent Cervical Cancer. Int J Radiat Oncol Biol Phys. 2017:98(5):1124-31.

3. Koh WJ, Greer BE, Abu-Rustum NR, Apte SM, Campos SM, Chan J, et al. Cervical cancer. J Natl Compr Canc Netw. 2013;11(3):320-43

4. Kim JY, Byun SJ, Kim YS, Nam JH. Disease courses in patients with residual tumor following concurrent chemoradiotherapy for locally advanced cervical cancer. Gynecol Oncol. 2017;144(1):34-9.

5. Schwarz JK, Siegel BA, Dehdashti F, Grigsby PW. Association of posttherapy positron emission tomography with tumor response and survival in cervical carcinoma. JAMA. 2007;298(19):2289-95.

6. Choi J, Kim HJ, Jeong YH, Lee JH, Cho A, Yun M, et al. The Role of (18) F-FDG PET/CT in Assessing Therapy Response in Cervix Cancer after Concurrent Chemoradiation Therapy. Nucl Med Mol Imaging. 2014:48(2):130-6

7. Siva S, Deb S, Young RJ, Hicks RJ, Callahan J, Bressel M, et al. (1)(8)F-FDG PET/CT following chemoradiation of uterine cervix cancer provides powerful prognostic stratification independent of HPV status: a prospective cohort of 105 women with mature survival data. Eur J Nucl Med Mol Imaging. 2015;42(12):1825-32.

8. Lee SI, Catalano OA, Dehdashti F. Evaluation of gynecologic cancer with MR imaging, 18F-FDG PET/CT, and PET/MR imaging. J Nucl Med. 2015;56(3):436-43.

9. Mongula J, Slangen B, Lambregts D, Bakers F, Mahesh S, Lutgens L, et al. Predictive criteria for MRI-based evaluation of response both during and after radiotherapy for cervical cancer. J Contemp Brachytherapy. 2016;8(3):181-8.

10. Vincens E, Balleyquier C, Rey A, Uzan C, Zareski E, Gouy S, et al. Accuracy of magnetic resonance imaging in predicting residual disease in patients treated for stage IB2/II cervical carcinoma with chemoradiation therapy : correlation of radiologic findings with surgicopathologic results. Cancer. 2008;113(8):2158-65.

11. Grueneisen J, Beiderwellen K, Heusch P, Gratz M, Schulze-Hagen A, Heubner M, et al. Simultaneous positron emission tomography/magnetic resonance imaging for whole-body staging in patients with recurrent gynecological malignancies of the pelvis: a comparison to whole-body magnetic resonance imaging alone. Invest Radiol. 2014;49(12):808-15.

12. Beiderwellen K, Grueneisen J, Ruhlmann V, Buderath P, Aktas B, Heusch P, et al. [(18)F]FDG PET/MRI vs. PET/CT for whole-body staging in patients with recurrent malignancies of the female pelvis: initial results. Eur J Nucl Med Mol Imaging. 2015;42(1):56-65.

13. DeLong ER, DeLong DM, Clarke-Pearson DL. Comparing the areas under two or more correlated receiver operating characteristic curves: a nonparametric approach. Biometrics. 1988;44(3):837-45.

14. Levy A, Caramella C, Chargari C, Medjhoul A, Rey A, Zareski E, et al. Accuracy of diffusion-weighted echo- 
planar MR imaging and ADC mapping in the evaluation of residual cervical carcinoma after radiation therapy. Gynecol Oncol. 2011;123(1):110-5.

15. Grueneisen J, Beiderwellen K, Heusch P, Buderath P, Aktas B, Gratz M, et al. Correlation of standardized uptake value and apparent diffusion coefficient in integrated whole-body PET/MRI of primary and recurrent cervical cancer. PLoS One. 2014:9(5):e96751.

16. Brandmaier P, Purz S, Bremicker K, Hockel M, Barthel H, Kluge R, et al. Simultaneous [18F]FDG-PET/MRI: Correlation of Apparent Diffusion Coefficient (ADC) and Standardized Uptake Value (SUV) in Primary and Recurrent Cervical Cancer. PLoS One. 2015;10(11):e0141684.

17. Ippolito D, Fior D, Trattenero C, Ponti ED, Drago S, Guerra L, et al. Combined value of apparent diffusion coefficient-standardized uptake value max in evaluation of post-treated locally advanced rectal cancer. World J Radiol. 2015;7(12):509-20. 


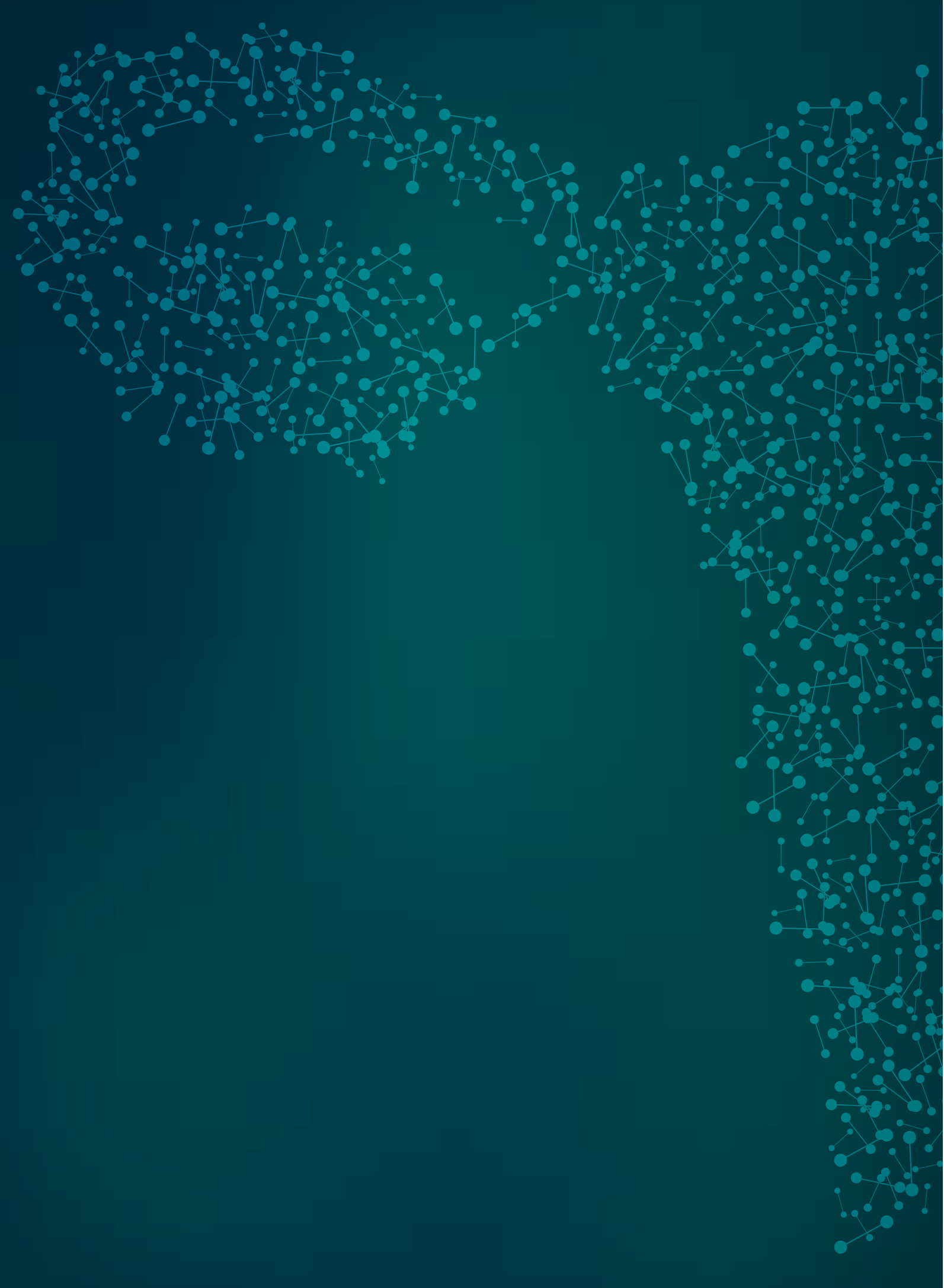

GENERAL DISCUSSION AND FUTURE PERSPECTIVES 
The aim of this thesis was twofold: Correct staging of cervical cancer (PART I) and evaluation of treatment response after CRT (PART II).

\section{PART I. MRI in early stage cervical carcinoma, prediction of parametrial invasion}

THE DIAGNOSTIC PERFORMANCE OF MRI FOR THE SUBJECTIVE ASSESSMENT OF PARAMETRIAL INVASION.

To date, staging of cervical cancer has been based on gynecologic examination, but MRI has shown to be superior to clinical examination. ${ }^{(1,2)}$ Hricak et al. already underlined the potential value of MRI for cervical cancer staging in the late eighties. However, only from 2018 onwards, both the ESGO and FIGO integrated MRI in their guidelines. ${ }^{(3,4)}$ Although, in Europe, approximately $74 \%$ of the gynecological oncology clinics use MRI for the assessment of early stage cervical cancer, the work-up and treatment still differs greatly between Gynecological Oncology clinics. ${ }^{(5)}$ MRI is valuable for the assessment of tumor diameter, locally advanced disease, and to some extent for parametrial invasion. ${ }^{(1,6)}$ Despite these new developments, still $13-25 \%$ of parametrial invasion is missed during staging. ${ }^{(1,7)}$ This results in additional treatment after surgery with increased patient morbidity. To improve the detection of parametrial invasion during the diagnostic work-up, we addressed the additional value of DWI for the assessment of parametrial invasion.

The fusion of T2W-MRI with DWI results in an increase in diagnostic performance for the assessment of parametrial invasion in clinical early-stage cervical carcinoma (Chapter 2). The positive predictive value significantly increased from $23-29 \%$ to $50 \%$. As postulated by other authors, a high positive predictive value is important for treatment planning ${ }^{(8)}$ However, the positive predictive value in our study was still only $50 \%$, which also means $50 \%$ of false positive findings based on DWI.(9) In addition, selection bias was unavoidable due to the fact that patients assigned to CRT by the multidisciplinary team (i.e. based on suspected parametrial invasion) could not be included because of the lack of pathological data from this group. The incorrect upstaging is an issue, radiologists tend to score more often false positively for parametrial invasion if large tumor diameters are seen on MRI. ${ }^{(8)}$ Tumor size on MRI was one of the main factors for incorrect upstaging to MRI based FIGO IIB stage $(9,6 \%$ of their cohort).

Two previous studies found a higher positive predictive value. ${ }^{(10,11)}$ They had a significant higher prevalence of parametrial invasion on pathology ( $12 \%$ versus $25 \%$ and $17 \%$ respectively). In contrast to our study, both these studies included patients with higher tumor stage (>FIGO IB 15\% versus 29\% and 67\% respectively). A recent metaanalysis supports our assumption as a tendency to increased sensitivity was found within studies including > IB2 cervical cancers. ${ }^{(2)}$ In our opinion FIGO > IB1 should still be treated predominantly with CRT to prevent additional treatment after surgery, as supported by recent guidelines. ${ }^{(4,12)}$ Therefore, at present our study represents the most challenging and clinical relevant group of patients; especially in these patients DWI could lead to an increase in the positive predictive value. ${ }^{(9)}$

Future studies will focus on identifying subgroups which benefit from the addition of DWI. One of the subgroups are patients within the FIGO IB1 group with tumors between $2-4 \mathrm{~cm}$. Tumors $<2 \mathrm{~cm}$ with favorable pathologic characteristics have an a priori low likelihood of parametrial invasion (0-0.6\%) whereas in tumors $>4 \mathrm{~cm}$ alternative treatment should be considered. ${ }^{(13,14)}$ In the near future, another subgroup may be patients with FIGO stage IB2, IIA or IIB receiving neo-adjuvant chemotherapy. Currently we are awaiting the results of the EORTC 55994 trial. In this trial, standard treatment (CRT) for stage IB2, IIA or IIB is compared to neo-adjuvant chemotherapy followed by radical hysterectomy. The latter group receives adjuvant radiotherapy if parametrium invasion is found in the histological specimen after surgery. In this group, correct assessment of parametrial invasion by DWI after chemotherapy is expected to have a major impact on the allocation to the correct patient tailored treatment. ${ }^{(15)}$

DWI could also have additional value in identifying low risk cervical cancers suitable for less radical surgery. ${ }^{(16-19)}$ The addition of DWI increased the negative predictive value for the assessment of parametrial invasion, although not yet statistically significant.(9) This is probably due to the already excellent negative predictive value of conventional MRI. However, even a small increase could be beneficial, especially if lower false negative findings will facilitate gynecologic oncologists to perform 'fertility sparing techniques' or 'simple' hysterectomy with lower patient morbidity compared to standard radical hysterectomy. An adequately powered cohort study to address this issue would be of interest.

RELIABLE MEASUREMENTS OF ADC AND ITS ROLE IN PREDICTING PARAMETRIAL AND/OR LYMPH NODE INVOLVEMENT

$A D C$ is an interesting parameter for the prediction of parametrial invasion, lymph node metastases, and therefore also survival. ${ }^{(20-23)}$ At the initiation of this study, standardized ADC measurement techniques were not available, despite the need for reliable and reproducible measurements. Literature on breast, prostate, gynecological and rectal 
tumors revealed conflicting results regarding observer variability with different $\mathrm{ROI}$ techniques. ${ }^{(24-27)}$ The literature also shows heterogenic use of techniques in cervical cancer. ${ }^{(20-23)}$ To assess ADC and its use in cervical cancer we initially had to assess the different ROI ADC measurement techniques in cervical cancer.

In chapter 3 we compared 2D region of interest (ROI) measurement techniques to 3D tumor analysis in the evaluation of different ADC parameters for cervical cancer, and found single $2 D$ ROI ADC measurements to be reliable for assessing the $A D C_{\text {Mean }}$ of early stage cervical cancer. As a consequence, our study supports both metaanalysis of previous literature and the use of less time-consuming ADC measurement techniques in future studies. ${ }^{(20-23)}$ In addition, both parameters $A D C_{\text {Min }}$ and $A D C_{\text {Max }}$ should be interpreted with caution, especially in heterogenic tumors. $A D C_{\text {Min }}$ and $A D C_{\text {Max }}$ are influenced by necrotic and normal cervical stroma respectively. ${ }^{(28)}$ The variability in $A D C_{\text {Min }}$ and $A D C_{\text {Max }}$ should be limited by using a method which avoids having to select the appropriate $2 \mathrm{D}-\mathrm{ROI}$. Total tumor delineation combined with histogram analysis might provide a solution in this respect. Histogram analysis is based on the selection of parts of a tumor with different diffusion characteristics, which results in more reliable and reproducible measurements as outlying values can be excluded. ${ }^{(29,30)}$ Moreover, histogram analysis can be used to assess the heterogeneity of a tumor, in other carcinoma's, tumor heterogeneity has shown to correlate with a poor prognosis. ${ }^{28,31,}$ 32) Secondly, histogram analysis can focus on the area within a tumor with the lowest $A D C$, in cervical cancer low $A D C_{\text {mean }}$ correlates with survival. ${ }^{(21-23)}$ Although histogram analysis appears to be a promising method, 3D delineation of the tumor, necessary for this analysis, remains rather time consuming. ${ }^{(28,33,34)}$

$A D C_{\text {Mean }}$ showed to be a more independent predictor of parametrial involvement than tumor diameter and subjective assessment of parametrial invasion on T2WMRI. Previous literature, based on retrospective cohorts support these findings. ${ }^{(20,21)}$ However, the relatively large proportion of IIB carcinoma (generally treated with CRT) may have increased the diagnostic performance of the test in those studies. In our opinion, ADC would be of particular value in those patients without clear parametrial invasion on T2W-MRI and/or clinical examination. Woo et al. questioned the value of $A D C_{\text {Mean }}$ when it was used as an adjunct to T2W-MRI with DWI of the parametrium. It remains questionable whether this study had sufficient power to justify this subgroup analysis. ${ }^{(35)}$ Despite methodological shortcomings their hypothesis is interesting: the association between ADC and parametrial involvement may in fact be due to the contribution of small MRI-invisible tumors. MRI-invisible tumors have a low potential to involve the parametria and as they are not visible, cervical stroma with a higher
$A D C_{\text {mean }}$ is measured. ${ }^{(8)}$ Our multivariate analyses (chapter 3) partially corrected for this issue. In addition to this, all patients with MRI-invisible tumors were excluded. In concordance with this remark, we do not support the use of $A D C_{\text {Mean }}$ as a sole MRI marker for parametrial involvement. However, we believe $A D C_{\text {Mean }}$ should be used as an adjunct to subjective assessment, especially when the radiologist is in doubt whether parametrial invasion is present. A recent study focused on this subgroup and surprisingly did not found an additional benefit of ADC ${ }^{(36)}$ They found remarkable high $A D C$ values in contrast to other studies. Therefore, we think that $A D C$ as an adjunct to subjective evaluation of parametrial invasion remains a question worth exploring. Future studies will focus on combining $A D C_{\text {Mean }}$ with $T 2 W-M R I$ and clinical risk factors to increase diagnostic performance for predicting parametrial involvement. Subsequently opening the window for tailored treatment to diminish the need for additional treatment after surgery. ${ }^{(20)}$ Another interesting question is the additional value of $A D C_{\text {Mean }}$ combined with fusion DWI for the assessment of parametrial invasion as proposed in Chapter 2. We hypothesize that the combination of the latter two will result in an increase in diagnostic performance.

In line with other studies, $A D C_{\text {Mean }}$ was able to predict lymph node metastases to some extent (Chapter 3). ${ }^{(21,23,37)}$ There are some biological grounds for these findings; $A D C_{\text {Mean }}$ is correlated with LVSI, and LVSI at the surgical specimen is a known risk factor for lymph node metastases and disease recurrence. (4, 21, 22) Integration of multiple techniques and parameters will prove to be inevitable to increase the diagnostic performance for the prediction of lymph node metastases. T2W-MRI is dependent on size and architecture and therefore misses small metastases. ${ }^{(38)} \mathrm{DWI}$ is hampered by diffusion restriction of normal lymphatic tissue but showed potential. ${ }^{(39)}$ PET-CT could be preferred to DWI due to high specificity, however sensitivity remains poor $(68 \%)$. ${ }^{(40)}$ Perhaps in the future, $A D C_{\text {Mean }}$ could be incorporated in a model for the prediction of lymph node metastases.

In conclusion, we support further research focusing on integrating multiple risk factors in predictive models. These models should provide individual risk assessments on parametrial invasion, lymph node metastasis, and the need for additional treatment after surgery. The individual percentages will support both gynecologic oncologists and patients for choosing the correct treatment option after patient counseling. Second to models based on regression analysis, machine learning based on Bayesian statistics is worth exploring. ${ }^{(41)}$ Bayesian models are able to incorporated existing evidence, statistics from regression models and multiple patient characteristics to make personalized risk assessments. Moreover, these models can also be updated 
by new data (machine based learning), making it a flexible model more true to the current individual patient situation. ${ }^{(41)}$ Future studies should also take more advanced MRI based biomarkers in to account; radiomics could very well be the future. ${ }^{(42)}$ However, we should not forget to explore the biological and molecular background of ADC. Preliminary studies in other cancer types showed ADC to be associated with angiogenic, hypoxic and immunologic markers. ${ }^{(43-46)} \mathrm{A}$ better understanding of the background will help us to understand the potential of ADC and to guide future research
PART II. MR and PET imaging for predicting response for locally advanced cervical carcinoma

\section{MR AND PET IMAGING FOR LOCALLY ADVANCED CERVICAL CARCINOMA}

Assessing the difference between viable residual carcinoma after (C)RT and radiationinduced changes like fibrosis remains challenging. ${ }^{(47)}$ The presence of residual disease is a significant predictor of patient survival. Because $30-40 \%$ of patients with isolated local residual disease can be salvaged with surgery, early detection could benefit this patient group. ${ }^{(48-50)}$ The correct selection of patients is essential as surgery is only beneficial in the subgroup of patients with isolated local residual disease. The majority of patients with complete response to RT will not benefit from additional surgery. ${ }^{(51,}$ 52) Subjective analyses of T2W-MRI for depicting local residual disease after RT show a low diagnostic performance predominantly due to false positive findings. ${ }^{(53,54)}$ Despite efforts by several research groups to improve the MRI evaluation, no unambiguous recommendations can be made to date. ${ }^{(6)}$

\section{IMAGING CRITERIA PREDICTIVE FOR RESIDUALTUMOR AFTER CHEMORADIATION} THERAPY

The use of objective MRI criteria improved the interobserver agreement and the diagnostic performance of the less experienced reader for assessment of residual disease after RT (Chapter 4). Our study highlights the observer dependency of subjective evaluation of response after RT on conventional T2W MR images. This is supported by the literature as a broad range of different values for diagnostic accuracy are reported. $(3,53,55,56)$ We therefore support the use of 'objective' MR criteria with higher interobserver agreement, keeping in mind that the use of 'iso-intensity' on T2W-MRI as a stand-alone criterion performs only moderate-average (AUC: 0.79, Chapter 4). The combination of 'iso-intensity' with two other criteria could improve the assessment of residual disease, provided that this regression model has to be validated.

It is important to notice that timing of MRI after RT is essential. In Chapter 4 results obtained at the last brachy application were rather poor compared to the evaluation 3 months after RT. We therefore hypothesize that especially early radiation effects as edema and inflammation hamper MRI evaluation. ${ }^{(57)}$ Our findings are supported by the literature; studies assessing residual disease on MRI within 6-8 week's report more false positive findings, supposedly due to post-RT effects mimicking residual tumor on MRI. ${ }^{(53,58)} \mathrm{A}$ small sequential MRI study showed a decrease in false positive results if MRI was evaluated 3 months compared to 1 month or directly after RT. (56) 
Chapter $\mathbf{4}$ also showed that the evaluation of MRIs obtained during RT could assist in the evaluation after treatment. In the subset of patients with available brachytherapy scans, the expert reader significantly improved the diagnostic performance for subjectively depicting residual cervical cancer. This suggests that the readers found the additional information provided by the BCT MR images very useful during their final assessment of the treatment response. This can be explained by the fact that volume regression over time can be observed more accurately on consecutive scans. This is in concordance with our findings in chapter 5 and previous literature. ${ }^{(59)}$ To date, MRI has not been integrated in the standard follow-up after RT. We hope that the combination of 'objective criteria', the availability of BCT MR images and new developments as DWI will improve the diagnostic performance for depicting residual tumor.

In studies assessing cervical cancer response after CRT, the existence of microscopic disease remains a major issue. There is no method available to discriminate between microscopic disease still in regression due to delayed radiation effects or foci of residual / recurrent disease. ${ }^{(60)}$ In contrast to our study, there are studies who performed standard surgery after CRT to assess the presence of residual disease (mostly within 6-8 weeks). ${ }^{(58)}$ Two-three year follow-up is preferable as a reference standard, as time will show the differentiation between non-active and active microscopic tumor cells after CRT. A large prospective study showed significant differences in survival after CRT followed by completion surgery between patients with no evidence of disease/ microscopic disease and especially macroscopic disease. ${ }^{(60)}$ Therefore, we support the use of follow-up as reference standard for studies assessing cervical cancer response. In case of pathology as a reference standard, researchers should think carefully about considering microscopic tumor cells as residual cervical cancer.

Exploring the potential of DWI for the assessment of residual cervical cancer is of interest. ${ }^{(61)}$ Effective RT will result in tumor lysis, necrosis, reduced tissue cellularity, damaged cell membranes, and increased extracellular space. ${ }^{(62)}$ These changes increase the diffusion properties of the treated tissue in contrast to residual disease were diffusion restriction is maintained. ${ }^{(63)}$ As a consequence, an increased ADC is expected if CRT has been successfully administered ${ }^{(62)}$ Important to notice is that due to the 'T2 shine through' (tissue with extreme long T2 signal can mimic tissue with restricted water mobility) high b-value diffusion images should always be interpreted with the corresponding ADC map. ${ }^{(64)}$ Two studies showed the benefit of subjective assessment of DWI for the depiction of residual disease after RT ${ }^{(65,66)}$ Both studies used 'iso-intensity' or residual intermediate signal on T2W-MRI as the criteria for residual disease which, in our study has shown to be of mediocre diagnostic performance as a stand-alone criterion. ${ }^{(57)}$ In addition, MRI was evaluated 4 or 8 weeks after RT that potentially showed early radiation effects that mimics residual disease. The prospective multicenter trial by Thomeer et al. considered microscopic tumor at pathology as significant residual disease, follow-up of 1 year was sufficient (surrogate complete response), and brachytherapy MRI was not used to guide the assessment for residual cancer. ${ }^{(65)}$ The value of quantitative ADC was not shown in this study but might be of additional value to discriminate between responders and non-responders. (67) The PRICE 2 study highlighted the shortcomings of T2W evaluation directly after neoadjuvant CRT. ${ }^{(58)} A D C$ and DWI is suggested to have some value when high T2 signal intensity is found, as supported by another retrospective study. ${ }^{(58,68)}$ In both studies, surgery was performed early after neoadjuvant CRT, without distinction between the macro- and microscopic tumor subgroup. A DWI sequence has been performed prior and after CRT in our prospective cohort (2011-ongoing) and will be evaluated in future papers. Second, we will prospectively evaluate our MR criteria set and the effect of the availability of MRIs during brachytherapy as suggested in chapter 4

\section{PREDICTION OF RESPONSE TO RADIOTHERAPY BY ASSESSING VOLUME} MEASUREMENTS

Mayr et al. showed tumor volume regression during and after RT to predict tumor response to the administered treatment. ${ }^{(59,69-74)}$ The addition of chemotherapy and the introduction of MR guided (brachy) radiation therapy (BCT) by the GEC-ESTRO working group led to significant improved patient survival. ${ }^{(73,75)}$ 3D MR guided RT has led to individual patient treatment with more accurate dose administration to the target tumor volume, increasing local control (87-95\%) and reducing late radiation induced toxicity $(50-60 \%) .{ }^{(76)}$ Despite increase in local control still a significant amount of patients fail to respond to CRT. The EMBRACE I study showed local treatment failure in $6.5 \%$ of the patient cohort treated with MRI guided BCT.(77) Theoretically, patients with a high chance of local failure could be monitored carefully to have the option to perform surgery in case of isolated local residual disease.(48-50) In these patients prediction of response during CRT could be beneficial as additional option for these patients is to perform additional hysterectomy after CRT. This is supported by the EMBRACE study; $98 \%$ of local failure occurred within the clinical target volume with the majority in the high-risk volume, so near the original cervical cancer. ${ }^{(77)}$ Previous studies show some potential of volume reduction measurements after CRT. However, as shown by both other and our research group (unpublished data) not nearly as potent as volume reduction during CRT. ${ }^{(56,59,78)}$ Another benefit of assessing response to treatment during radiation is the possibility to perform radiation escalation of poor responding tumor bearing regions. In case of large radio insensitive foci, it is even possible to allocate 
patients to surgery during radiation treatment to minimalize the radiation dose. The latter has not been done but neo-adjuvant CRT has been performed by some research groups with acceptable survival and morbidity. ${ }^{(79-81)}$ However, a randomized controlled trial comparing neo-adjuvant CRT followed by surgery, with standard CRT followed by BT did not show differences in survival with approximately $26 \%$ complication risk. ${ }^{(82)}$ Dose de-escalation for good responders is another promising option, thus reducing radiation burden for the patient, potentially decreasing radiation induced toxicity which still is around $50-60 \% .{ }^{(76)}$

Absolute volume measurements as well as volume regression at BCT are promising predictors of local residual disease (Chapter 5). Our results are in line with previous literature which showed initial tumor volume and volume regression at the end of EBRT (corresponding with the first BCT) to predict local treatment failure. ${ }^{(59,70)}$ In these studies, volumetric analyses were performed without the BCT applicator in situ, necessitating an additional MRI scan. Our results show that volumetric analyses on MRI images performed for RT planning with the BCT device in situ are feasible, lowering the threshold for implementation in clinical practice. Another large retrospective study assessed volume regression during BCT and showed comparable cut-offs and results to our study. ${ }^{\left({ }^{(3)}\right)}$ We have to admit that the evaluation at the first BCT might be preferable, as dose de-escalation for good responders compared to dose escalation for poor responders might be an interesting option worth exploring in future studies. ${ }^{(83)}$ Even earlier prediction of local tumor control after 2 weeks of EBRT has been attempted, but volume parameters were not nearly as potent as at the time of the first BCT. $(59,70)$

Multiple groups tried to investigate DWI parameters early in the course of CRT as a change in functional tumor parameters is expected to precede volume reduction. ${ }^{(61,84)}$ However, to the best of our knowledge, no studies showed biomarkers strong enough to be used as a stand-alone criterion. ${ }^{\left({ }^{85}\right)}$ Perhaps integration in a predictive model, histogram analysis, radiomics, and/or the measurement of functional tumor volume will prove to be the solution.

To date, in our tertiary center all patients have surveillance MRI and PET-CT or PETMR 3 months after treatment. As the majority of the patients will have a complete response to CRT, stratification of patients in different risk groups could be beneficial. In contrast to low-risk patients, high risk patients might benefit from surveillance imaging to detect patients with residual disease eligible for additional surgery. Ultimately, we would like to predict the response to treatment during RT to tailor patient followup and treatment regime accordingly. Future perspectives as residual functional tumor volume either by DW-MRI or PET, predictive models, histogram analysis and/or radiomics, might prove to be the solution.

\section{${ }^{18}$ F-FDG POSITRON EMISSION TOMOGRAPHY - MAGNETIC RESONANCE IMAGING (PET-MRI) FOR RESPONSE ASSESSMENT AFTER CHEMO-RADIATION THERAPY OF CERVICAL CARCINOMA.}

Tumor glucose uptake as assessed by PET could diminish false positive results by differentiating between active (glucose-uptake) residual cervical cancer and radiationinduced changes/non-active residual cancer in regression after RT. Our pilot study (Chapter 6) suggests combined PET-MR evaluation for assessing treatment response after RT; the addition of PET to MRI diminished both false positive and false negative MRI findings. In concordance with our findings, Gruneisen et al. showed PET-MR to be superior to whole-body MRI for detecting suspected recurrence while improving diagnostic confidence of the observers. ${ }^{(86)}$ In addition, in our study, the PET component was able to detect metastatic disease that significantly affected the policy for these patients, as previously suggested by other studies assessing PET-CT. ${ }^{(87,88)}$ This second finding is important as approximately $50 \%$ of patients with local residual disease have concurrently distant metastasis. ${ }^{(77)}$ These patients will not benefit from salvage surgery.

Despite a limited amount of data, the NCCN guidelines advice restaging at 3-6 months after CRT with PET-CT. These recommendations are based on two cohort studies showing PET-FDG combined with CT 3 months after CRT have high potential for predicting long term survival. ${ }^{(87,88)}$ Furthermore, PET-CT results were able to guide additional salvage therapy achieving reasonable survival. ${ }^{(87,88)}$ Recently, another research group confirmed the value of PET-CT 3 months after CRT, additionally showing metabolic tumor volume to be an interesting parameter. ${ }^{\left({ }^{(8)}\right)}$ This group stated that the timing of the PET-CT scan could be a major limitation. Despite the NCCN advice to perform restaging at 3-6 months after treatment, all previous mentioned studies performed PET-CT up to 12 months after CRT. To the best of our knowledge there is no literature available assessing the timing of the PET after radiation therapy. This remains an interesting question as early evaluation could result in false positive findings due to FDG uptake in lesions still in regression. Therefore, recommended timing for PET appears to be 3 months after CRT. ${ }^{90}$

Based on the existing literature and our pilot study both PET-CT and PET-MR are worth exploring after CRT. Due to our small patient population, we were not able to address the changes in functional PET and MRI parameters. Changes after treatment of $A D C_{\text {Mea }}$ and SUV could be of interest, especially if both parameters prove to be of independent 
value. Combining both PET and MRI data could be of value in the answer for some of these questions. Additionally, the amount of functional tumor volume measured by either SUV or ADC could be of interest as this probably reflects the active part of the residual cervical tumor. Future research will have to consist of a larger cohort to validate our findings and to test the aforementioned new hypotheses.

Hopefully, PET-MR proves to be capable of accurately assessing local residual cervical cancer while excluding metastatic disease. As a result, identification of patients with isolated residual disease who may be salvaged by surgery becomes an option, without exposing a large number of patients to futile treatments. The existing cohort studies lack a control group without imaging, because of possible lead-time effects, survival benefit can be suggested but not proven. Therefore, to address the possible survival benefit of PET-MR imaging and follow-up strategies, a random controlled trial is the next step. We believe that PET-MR imaging eventually will uphold its promise to select those patients benefiting from salvage surgery without exposing a substantial number of patients to futile surgery.

\section{REFERENCE}

1. Thomeer MG, Gerestein C, Spronk S, van Doorn HC, van der Ham E. Hunink MG. Clinical examination versus magnetic resonance imaging in the pretreatment staging of cervical carcinoma: systematic review and meta-analysis. Eur Radiol. 2013;23(7):2005-18

2. Woo S, Suh $\mathrm{CH}$, Kim SY, Cho JY, Kim SH. Magnetic resonance imaging for detection of parametrial invasion in cervical cancer: An updated systematic review and meta-analysis of the literature between 2012 and 2016. Eur Radiol. 2017.

3. Hricak H. MRI of the female pelvis: a review. AJR Am J Roentgenol. 1986;146(6):1115-22.

4. Cibula D, Potter R, Planchamp F, Avall-Lundqvist E, Fischerova D, Haie Meder C, et al. The European Society of Gynaecological Oncology/European Society for Radiotherapy and Oncology/European Society of Pathology guidelines for the management of patients with cervical cancer. Radiother Oncol. 2018;127(3):404-16.

5. Dostalek L, Avall-Lundqvist E, Creutzberg CL, Kurdiani D, Ponce J, Dostalkova I, et al. ESGO Survey on Current Practice in the Management of Cervical Cancer. Int J Gynecol Cancer. 2018;28(6):1226-31.

6. Zhang J, Tian W, Bu X, Wang X, Tian F, Wu L. Diagnostic significance of magnetic resonance imaging in patients with cervical cancer after brachytherapy: a meta-analysis. Acta Radiol. 2018:284185118791199.

7. Kato T, Takashima A, Kasamatsu T, Nakamura K, Mizusawa J, Nakanishi T, et al. Clinical tumor diameter and prognosis of patients with FIGO stage IB1 cervical cancer (JCOG0806-A). Gynecol Oncol. 2015;137(1):34-

8. Roh HJ, Kim KB, Lee JH, Kim HJ, Kwon YS, Lee SH. Early Cervical Cancer: Predictive Relevance of Preoperative 3-Tesla Multiparametric Magnetic Resonance Imaging. Int J Surg Oncol. 2018;2018:9120753.

9. Mongula JE, Bakers FCH, Mihl C, van Gorp T, Kruitwagen R, Slangen BFM. Assessment of parametrial invasion of cervical carcinoma, the role of T2-weighted MRI and diffusion weighted imaging with or without fusion. Clin Radiol. 2019;74(10):790-6.

10. Qu JR, Qin L, Li X, Luo JP, Li J, Zhang HK, et al. Predicting Parametrial Invasion in Cervical Carcinoma (Stages IB1, IB2, and IIA): Diagnostic Accuracy of T2-Weighted Imaging Combined With DWI at 3 T. AJR Am J Roentgenol. 2018;210(3):677-84.

11. Park JJ, Kim CK, Park SY, Park BK. Parametrial invasion in cervical cancer: fused T2-weighted imaging and high-b-value diffusion-weighted imaging with background body signal suppression at 3 T. Radiology. 2015;274(3):734-41.

12. Katanyoo K, Praditsitthikorn N, Tangjitgamol S, Manusirivithaya S, Supawattanabodee B. Cost-utility analysis of treatments for stage IB cervical cancer. J Gynecol Oncol. 2014:25(2):97-104.

13. Vranes B, Milenkovic S, Radojevic M, Soldatovic I, Kesic V. Risk of Parametrial Spread in Small Stage I Cervical Carcinoma: Pathology Review of 223 Cases With a Tumor Diameter of $20 \mathrm{~mm}$ or Less. Int J Gynecol Cancer. 2016;26(2):416-21

14. Schmeler KM, Frumovitz M, Ramirez PT. Conservative management of early stage cervical cancer: is there a role for less radical surgery? Gynecol Oncol. 2011;120(3):321-5. 
15. Qendri V, Schurink-Van 't Klooster TM, Bogaards JA, Berkhof J. Ten years of HPV vaccination in the Netherlands: current evidence and future challenges in HPV-related disease prevention. Expert Rev Vaccines. 2018;17(12):1093-104.

16. Lee JY, Youm J, Kim JW, Cho JY, Kim MA, Kim TH, et al. Identifying a low-risk group for parametrial involvement in microscopic Stage IB1 cervical cancer using criteria from ongoing studies and a new MRI criterion. BMC Cancer. 2015;15:167

17. Crosbie EJ, Einstein MH, Franceschi S, Kitchener HC. Human papillomavirus and cervical cancer. Lancet. 2013;382(9895):889-99

18. Cohen PA, Jhingran A, Oaknin A, Denny L. Cervical cancer. Lancet. 2019:393(10167):169-82.

19. Group FIS. Quadrivalent vaccine against human papillomavirus to prevent high-grade cervical lesions. N Engl J Med. 2007;356(19):1915-27.

20. Park JJ, Kim CK, Park SY, Park BK, Kim B. Value of diffusion-weighted imaging in predicting parametrial invasion in stage IA2-IIA cervical cancer. Eur Radiol. 2014;24(5):1081-8.

21. Nakamura K, Joja I, Nagasaka T, Fukushima C, Kusumoto T, Seki N, et al. The mean apparent diffusion coefficient value (ADCmean) on primary cervical cancer is a predictive marker for disease recurrence. Gynecol Oncol. 2012:127(3):478-83.

22. Zhou G, Chen X, Tang F, Zhou J, Wang Y, Wang Z. The Value of Diffusion-Weighted Imaging in Predicting the Prognosis of Stage IB-IIA Cervical Squamous Cell Carcinoma After Radical Hysterectomy. Int J Gynecol Cancer. 2016;26(2):361-6.

23. Micco M, Vargas HA, Burger IA, Kollmeier MA, Goldman DA, Park KJ, et al. Combined pre-treatment MRI and 18F-FDG PET/CT parameters as prognostic biomarkers in patients with cervical cancer. Eur J Radiol. 2014;83(7):1169-76.

24. Tamada T, Huang C, Ream JM, Taffel M, Taneja SS, Rosenkrantz AB. Apparent Diffusion Coefficient Values of Prostate Cancer: Comparison of 2D and 3D ROIs. AJR Am J Roentgenol. 2018;210(1):113-7.

25. Inoue C, Fujii S, Kaneda S, Fukunaga T, Kaminou T, Kigawa J, et al. Apparent diffusion coefficient (ADC) measurement in endometrial carcinoma: effect of region of interest methods on ADC values. J Magn Reson Imaging. 2014;40(1):157-61.

26. Lambregts DM, Beets GL, Maas M, Curvo-Semedo L, Kessels AG, Thywissen T, et al. Tumour ADC measurements in rectal cancer: effect of ROI methods on ADC values and interobserver variability. Eur Radiol. 2011;21(12):2567-74

27. Mukuda N, Fujii S, Inoue C, Fukunaga T, Tanabe Y, Itamochi H, et al. Apparent diffusion coefficient (ADC) measurement in ovarian tumor: Effect of region-of-interest methods on ADC values and diagnostic ability. J Magn Reson Imaging. 2016;43(3):720-5.

28. Guan Y, Shi H, Chen Y, Liu S, Li W, Jiang Z, et al. Whole-Lesion Histogram Analysis of Apparent Diffusion Coefficient for the Assessment of Cervical Cancer. J Comput Assist Tomogr. 2016;40(2):212-7.

29. Schob S, Meyer HJ, Pazaitis N, Schramm D, Bremicker K, Exner M, et al. ADC Histogram Analysis of Cervical Cancer Aids Detecting Lymphatic Metastases-a Preliminary Study. Mol Imaging Biol. 2017.

30. Heo SH, Shin SS, Kim JW, Lim HS, Jeong YY, Kang WD, et al. Pre-treatment diffusion-weighted MR imaging for predicting tumor recurrence in uterine cervical cancer treated with concurrent chemoradiation: value of histogram analysis of apparent diffusion coefficients. Korean J Radiol. 2013;14(4):616-25.

31. Ren JL, Yuan Y, Li XX. Shi YQ. Tao XF. Histogram analysis of apparent diffusion coefficient maps in the prognosis of patients with locally advanced head and neck squamous cell carcinoma: Comparison of different region of interest selection methods. Eur J Radiol. 2018;106:7-13.

32. Pham TT, Liney GP, Wong K, Barton MB. Functional MRI for quantitative treatment response prediction in locally advanced rectal cancer. Br J Radiol. 2017:90(1072):20151078

33. Downey K, Riches SF, Morgan VA, Giles SL, Attygalle AD, Ind TE, et al. Relationship between imaging biomarkers of stage I cervical cancer and poor-prognosis histologic features: quantitative histogram analysis of diffusion-weighted MR images. AJR Am J Roentgenol. 2013;200(2):314-20.

34. Lin Y, Li H, Chen Z, Ni P, Zhong Q, Huang H, et al. Correlation of histogram analysis of apparent diffusion coefficient with uterine cervical pathologic finding. AJR Am J Roentgenol. 2015;204(5):1125-31.

35. Woo S, Kim SY, Cho JY, Kim SH. Apparent diffusion coefficient for prediction of parametrial invasion in cervical cancer: a critical evaluation based on stratification to a Likert scale using T2-weighted imaging. Radiol Med. 2017.

36. Song J, Hu Q, Ma Z, Zhang J, Chen T. Value of diffusion-weighted and dynamic contrast-enhanced MR in predicting parametrial invasion in cervical stromal ring focally disrupted stage IB-IIA cervical cancers. Abdom Radiol (NY). 2019:44(9):3166-74

37. Kuang F, Ren J, Zhong Q, Liyuan F, Huan Y, Chen Z. The value of apparent diffusion coefficient in the assessment of cervical cancer. Eur Radiol. 2013;23(4):1050-8.

38. Dappa E, Elger T, Hasenburg A, Duber C, Battista MJ, Hotker AM. The value of advanced MRI techniques in the assessment of cervical cancer: a review. Insights Imaging. 2017;8(5):471-81

39. Shen $G$, Zhou H, Jia Z, Deng H. Diagnostic performance of diffusion-weighted MRI for detection of pelvic metastatic lymph nodes in patients with cervical cancer: a systematic review and meta-analysis. $\mathrm{Br} \mathrm{J}$ Radiol. 2015;88(1052):20150063.

40. Gong Y, Wang Q, Dong L, Jia Y, Hua C, Mi F, et al. Different imaging techniques for the detection of pelvic lymph nodes metastasis from gynecological malignancies: a systematic review and meta-analysis. Oncotarget. 2017;8(8):14107-25

41. Arora P, Boyne D, Slater JJ, Gupta A, Brenner DR, Druzdzel MJ. Bayesian Networks for Risk Prediction Using Real-World Data: A Tool for Precision Medicine. Value Health. 2019;22(4):439-45.

42. Wang $T$, Gao $T$, Yang J, Yan $X$, Wang $Y$, Zhou $X$, et al. Preoperative prediction of pelvic lymph nodes metastasis in early-stage cervical cancer using radiomics nomogram developed based on T2-weighted MRI and diffusion-weighted imaging. Eur J Radiol. 2019;114:128-35.

43. Meng X, Li H, Kong L, Zhao X, Huang Z, Zhao H, et al. MRI In rectal cancer: Correlations between MRI features and molecular markers Ki-67, HIF-1alpha, and VEGF. J Magn Reson Imaging. 2016:44(3):594-

44. Huang $Z$, X X X, Meng $X$, Hou Z, Liu F, Hua Q, et al. Correlations between ADC values and molecular markers of Ki-67 and HIF-1alpha in hepatocellular carcinoma. Eur J Radiol. 2015;84(12):2464-9. 
45. Surov A, Meyer HJ, Hohn AK, Sabri O, Purz S. Combined Metabolo-Volumetric Parameters of (18)F-FDGPET and MRI Can Predict Tumor Cellularity, Ki67 Level and Expression of HIF 1alpha in Head and Neck Squamous Cell Carcinoma: A Pilot Study. Transl Oncol. 2018;12(1):8-14.

46. Sersa I, Bajd F, Savarin M, Jesenko T, Cemazar M, Sersa G. Multiparametric High-Resolution MRI as a Tool for Mapping of Hypoxic Level in Tumors. Technol Cancer Res Treat. 2018:17:1533033818797066.

47. Elit L, Kennedy EB, Fyles A, Metser U. Follow-up for cervical cancer: a Program in Evidence-Based Care systematic review and clinical practice guideline update. Curr Oncol. 2016;23(2):109-18

48. Peiretti M, Zapardiel I, Zanagnolo V, Landoni F, Morrow CP, Maggioni A. Management of recurrent cervical cancer: a review of the literature. Surg Oncol. 2012;21(2):e59-66.

49. Kim TH, Kim MH, Kim BJ, Park SI, Ryu SY, Cho CK. Prognostic Importance of the Site of Recurrence in Patients With Metastatic Recurrent Cervical Cancer. Int J Radiat Oncol Biol Phys. 2017:98(5):1124-31.

50. Hong JH, Tsai CS, Lai CH, Chang TC, Wang CC, Chou HH, et al. Recurrent squamous cell carcinoma of cervix after definitive radiotherapy. Int J Radiat Oncol Biol Phys. 2004;60(1):249-57.

51. Keys HM, Bundy BN, Stehman FB, Okagaki T, Gallup DG, Burnett AF, et al. Radiation therapy with and without extrafascial hysterectomy for bulky stage IB cervical carcinoma: a randomized trial of the Gynecologic Oncology Group. Gynecol Oncol. 2003;89(3):343-53.

52. Kokka F, Bryant A, Brockbank E, Powell M, Oram D. Hysterectomy with radiotherapy or chemotherapy or both for women with locally advanced cervical cancer. Cochrane Database Syst Rev. 2015(4):CD010260.

53. Vincens E, Balleyguier C, Rey A, Uzan C, Zareski E, Gouy S, et al. Accuracy of magnetic resonance imaging in predicting residual disease in patients treated for stage IB2/II cervical carcinoma with chemoradiation therapy : correlation of radiologic findings with surgicopathologic results. Cancer. 2008:113(8):2158-65.

54. Kim JY, Byun SJ, Kim YS, Nam JH. Disease courses in patients with residual tumor following concurrent chemoradiotherapy for locally advanced cervical cancer. Gynecol Oncol. 2017;144(1):34-9.

55. Manfredi R, Maresca G, Smaniotto D, Greggi S, Andrulli D, Rabitti C, et al. Cervical cancer response to neoadjuvant therapy: MR imaging assessment. Radiology. 1998;209(3):819-24.

56. Hatano K, Sekiya Y, Araki H, Sakai M, Togawa T, Narita Y, et al. Evaluation of the therapeutic effect of radiotherapy on cervical cancer using magnetic resonance imaging. Int J Radiat Oncol Biol Phys. 1999;45(3):639-44.

57. Mongula J, Slangen B, Lambregts D, Bakers F, Mahesh S, Lutgens L, et al. Predictive criteria for MRI-based evaluation of response both during and after radiotherapy for cervical cancer. J Contemp Brachytherapy. 2016;8(3):181-8.

58. Gui B, Micco M, Valentini AL, Cambi F, Pasciuto T, Testa A, et al. Prospective multimodal imaging assessment of locally advanced cervical cancer patients administered by chemoradiation followed by radical surgery-the "PRICE" study 2: role of conventional and DW-MRI. Eur Radiol. 2018

59. Wang JZ, Mayr NA, Zhang D, Li K, Grecula JC, Montebello JF, et al. Sequential magnetic resonance imaging of cervical cancer: the predictive value of absolute tumor volume and regression ratio measured before, during, and after radiation therapy. Cancer. 2010;116(21):5093-101.

60. Ferrandina G, Margariti PA, Smaniotto D, Petrillo M, Salerno MG, Fagotti A, et al. Long-term analysis of clinical outcome and complications in locally advanced cervical cancer patients administered concomitant chemoradiation followed by radical surgery. Gynecol Oncol. 2010;119(3):404-10.

61. Levy A, Caramella C. Chargari C. Medjhoul A, Rey A. Zareski E, et al. Accuracy of diffusion-weighted echoplanar MR imaging and ADC mapping in the evaluation of residual cervical carcinoma after radiation therapy. Gynecol Oncol. 2011;123(1):110-5

62. Chen J, Zhang Y, Liang B, Yang Z. The utility of diffusion-weighted MR imaging in cervical cancer. Eur J Radiol. 2010;74(3):e101-6.

63. Bae JM, Kim CK, Park JJ, Park BK. Can diffusion-weighted magnetic resonance imaging predict tumor recurrence of uterine cervical cancer after concurrent chemoradiotherapy? Abdom Radiol (NY). 2016;41(8):1604-10.

64. Sala E, Rockall AG, Freeman SJ, Mitchell DG, Reinhold C. The added role of MR imaging in treatment stratification of patients with gynecologic malignancies: what the radiologist needs to know. Radiology. 2013;266(3):717-40.

65. Thomeer MG, Vandecaveye V, Braun L, Mayer F, Franckena-Schouten M, de Boer P, et al. Evaluation of T2-W MR imaging and diffusion-weighted imaging for the early post-treatment local response assessment of patients treated conservatively for cervical cancer: a multicentre study. Eur Radiol. 2018.

66. Park JJ, Kim CK, Park BK. Prediction of disease progression following concurrent chemoradiotherapy for uterine cervical cancer: value of post-treatment diffusion-weighted imaging. Eur Radiol. 2016:26(9):3272-

67. Schreuder SM, Lensing R, Stoker J, Bipat S. Monitoring treatment response in patients undergoing chemoradiotherapy for locally advanced uterine cervical cancer by additional diffusion-weighted imaging: A systematic review. J Magn Reson Imaging. 2015;42(3):572-94

68. Jalaguier-Coudray A, Villard-Mahjoub R, Delouche A, Delarbre B, Lambaudie E, Houvenaeghel G, et al. Value of Dynamic Contrast-enhanced and Diffusion-weighted MR Imaging in the Detection of Pathologic Complete Response in Cervical Cancer after Neoadjuvant Therapy: A Retrospective Observational Study. Radiology. 2017;284(2):432-42.

69. Mayr NA, Magnotta VA, Ehrhardt JC, Wheeler JA, Sorosky JI, Wen BC, et al. Usefulness of tumor volumetry by magnetic resonance imaging in assessing response to radiation therapy in carcinoma of the uterine cervix. Int J Radiat Oncol Biol Phys. 1996;35(5):915-24

70. Mayr NA, Wang JZ, Lo SS, Zhang D, Grecula JC, Lu L, et al. Translating response during therapy into ultimate treatment outcome: a personalized 4-dimensional MRI tumor volumetric regression approach in cervical cancer. Int J Radiat Oncol Biol Phys. 2010;76(3):719-27.

71. Nam H, Park W, Huh SJ, Bae DS, Kim BG, Lee JH, et al. The prognostic significance of tumor volume regression during radiotherapy and concurrent chemoradiotherapy for cervical cancer using MRI. Gynecol Oncol. 2007:107(2):320-5.

72. Eifel PJ, Winter K, Morris M, Levenback C, Grigsby PW, Cooper J, et al. Pelvic irradiation with concurrent chemotherapy versus pelvic and para-aortic irradiation for high-risk cervical cancer: an update of radiation therapy oncology group trial (RTOG) 90-01. J Clin Oncol. 2004:22(5):872-80. 
73. Harkenrider MM, Alite F, Silva SR, Small W, Jr. Image-Based Brachytherapy for the Treatment of Cervical Cancer. Int J Radiat Oncol Biol Phys. 2015;92(4):921-34.

74. Sturdza A, Potter R, Fokdal LU, Haie-Meder C, Tan LT, Mazeron R, et al. Image guided brachytherapy in locally advanced cervical cancer: Improved pelvic control and survival in RetroEMBRACE, a multicenter cohort study. Radiother Oncol. 2016;120(3):428-33.

75. Potter R, Georg P, Dimopoulos JC, Grimm M, Berger D, Nesvacil N, et al. Clinical outcome of protocol based image (MRI) guided adaptive brachytherapy combined with 3D conformal radiotherapy with or without chemotherapy in patients with locally advanced cervical cancer. Radiotherapy and oncology : journal of the European Society for Therapeutic Radiology and Oncology. 2011;100(1):116-23.

76. Potter R, Tanderup K, Kirisits C, de Leeuw A, Kirchheiner K, Nout R, et al. The EMBRACE II study: The outcome and prospect of two decades of evolution within the GEC-ESTRO GYN working group and the EMBRACE studies. Clin Transl Radiat Oncol. 2018:9:48-60

77. Schmid M. Local failures after radiochemotherapy and MR-image-guided brachytherapy in cervical cancer patients. . Radiother Oncol 2016;2017;123:S26.

78. Nam H, Park W, Huh SJ, Bae DS, Kim BG, Lee JH, et al. The prognostic significance of tumor volume regression during radiotherapy and concurrent chemoradiotherapy for cervical cancer using MRI. Gynecol Oncol. 2007:107(2):320-5

79. Ferrandina G, Gambacorta A, Gallotta V, Smaniotto D, Fagotti A, Tagliaferri L, et al. Chemoradiation with concomitant boosts followed by radical surgery in locally advanced cervical cancer: long-term results of the ROMA-2 prospective phase 2 study. Int J Radiat Oncol Biol Phys. 2014;90(4):778-85.

80. Rufini V, Collarino A, Calcagni ML, Meduri GM, Fuoco V, Pasciuto T, et al. The role of (18)F-FDG-PET/CT in predicting the histopathological response in locally advanced cervical carcinoma treated by chemoradiotherapy followed by radical surgery: a prospective study. Eur J Nucl Med Mol Imaging. 2019.

81. Couvreur K, Naert E, De Jaeghere E, Tummers P, Makar A, De Visschere P, et al. Neo-adjuvant treatment of adenocarcinoma and squamous cell carcinoma of the cervix results in significantly different pathological complete response rates. BMC Cancer. 2018;18(1):1101.

82. Cetina L, Gonzalez-Enciso A, Cantu D, Coronel J, Perez-Montiel D, Hinojosa J, et al. Brachytherapy versus radical hysterectomy after external beam chemoradiation with gemcitabine plus cisplatin: a randomized, phase III study in IB2-IIB cervical cancer patients. Ann Oncol. 2013;24(8):2043-7.

83. Schernberg A, Bockel S, Annede P, Fumagalli I, Escande A, Mignot F, et al. Tumor Shrinkage During Chemoradiation in Locally Advanced Cervical Cancer Patients: Prognostic Significance, and Impact for Image-Guided Adaptive Brachytherapy. Int J Radiat Oncol Biol Phys. 2018;102(2):362-72.

84. Kuang F, Yan Z, Wang J, Rao Z. The value of diffusion-weighted MRI to evaluate the response to radiochemotherapy for cervical cancer. Magn Reson Imaging. 2014;32(4):342-9.

85. Hameeduddin A, Sahdev A. Diffusion-weighted imaging and dynamic contrast-enhanced MRI in assessing response and recurrent disease in gynaecological malignancies. Cancer Imaging. 2015;15:3.

86. Grueneisen J, Beiderwellen K, Heusch P, Gratz M, Schulze-Hagen A, Heubner M, et al. Simultaneous positron emission tomography/magnetic resonance imaging for whole-body staging in patients with recurrent gynecological malignancies of the pelvis: a comparison to whole-body magnetic resonance imaging alone. Invest Radiol. 2014;49(12):808-15

87. Schwarz JK, Siegel BA, Dehdashti F. Grigsby PW. Association of posttherapy positron emission tomography with tumor response and survival in cervical carcinoma. JAMA. 2007;298(19):2289-95.

88. Siva S, Deb S, Young RJ, Hicks RJ, Callahan J, Bressel M, et al. (1)(8)F-FDG PET/CT following chemoradiation of uterine cervix cancer provides powerful prognostic stratification independent of HPV status: a prospective cohort of 105 women with mature survival data. Eur J Nucl Med Mol Imaging. 2015;42(12):1825-32

89. Lima GM, Matti A, Vara G, Dondi G, Naselli N, De Crescenzo EM, et al. Prognostic value of posttreatment (18)F-FDG PET/CT and predictors of metabolic response to therapy in patients with locally advanced cervical cancer treated with concomitant chemoradiation therapy: an analysis of intensity- and volumebased PET parameters. Eur J Nucl Med Mol Imaging. 2018;45(12):2139-46.

90. Kim YJ, Han S, Kim YS, Nam JH. Prognostic value of post-treatment (1)(8)F-fluorodeoxyglucose positron emission tomography in uterine cervical cancer patients treated with radiotherapy: a systematic review and meta-analysis. J Gynecol Oncol. 2019;30(5):e66. 


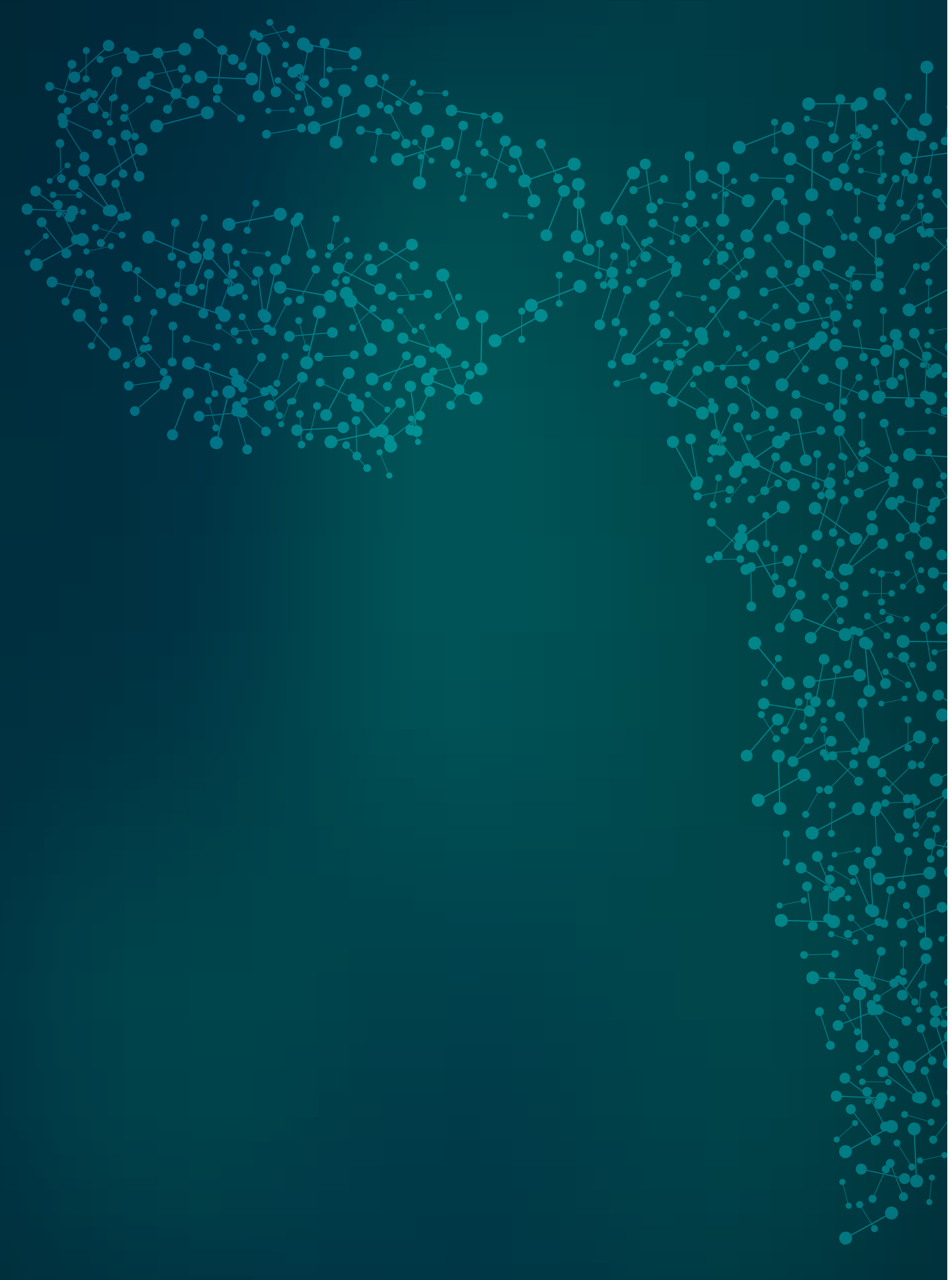

8|

SUMMARY 
In spite of extensive screening programs for pre-malignancy, cervical cancer is the third most common malignancy among women worldwide. Treatment is generally divided in surgery for early stage cervical cancer and chemoradiation (CRT) for locally advanced cervical cancer. Invasion of the adjacent parametrium is the most important turning point for the assignment to the right treatment. Staging has been traditionally performed by gynecological examination. The addition of MRI to the staging process significantly improves the assessment of the parametrium. However, assessing parametrial invasion on T2W-MRI is prone to false positive results; and still $13-25 \%$ of parametrial invasion is missed with conventional MRI. Patients with post-operative parametrial invasion receive additional (C)RT. Guidelines advocate to prevent surgery followed by (C)RT; more accurate staging could reduce triple modality treatment and therefore morbidity with comparable oncological outcome and less costs. Therefore, in PART I we addressed the value of new MRI techniques in early stage cervical carcinoma with emphasis on the prediction of parametrial invasion. We focused on the potential of diffusion weighted MRI (DWI). DWI reflects cell density by measuring water diffusion, cervical cancer has a higher cell density compared to normal cervical stroma or parametrium

Approximately $30 \%$ of all cervical cancer patients eventually die due to persistent or recurrent disease. In case of isolated local persistent disease after CRT, salvage surgery is an option with a reasonable 5 -year survival of $35-40 \%$; however, both morbidity and mortality (10\%) of the intervention are high. Therefore, PART II focused on MRI for prediction of local response for locally advanced cervical carcinoma.

\section{PART I. MRI IN EARLY STAGE CERVICAL CARCINOMA, PREDICTION OF}

\section{PARAMETRIAL INVASION}

In Chapter $\mathbf{2}$ we aimed to evaluate the diagnostic performance of DWI in addition to regular T2W sequences for the assessment of parametrial invasion. It was shown that the fusion of T2W-MRI with DWI results in an increase in diagnostic performance for the assessment of parametrial invasion in clinical early-stage cervical carcinoma. The positive predictive value for parametrial invasion significantly increased from 23-29\% to $50 \%$. Based on these data and the previous literature, DWI as an adjunct to T2W-MRI should be considered as a mandatory work-up tool for the assessment of parametrial invasion

In addition to a qualitative approach we also aimed to quantitatively evaluate the value of DWI for the pre-operative assessment of parametrial and lymph node involvement (Chapter 3). Secondly, we compared different measurement techniques to determine their reliability for assessing the qualitative derivative of DWI: Apparent diffusion coefficient (ADC). Single two-dimensional region of interest (ROI) ADC measurements were found to be reliable for assessing the mean $A D C\left(A D C_{\text {Mean }}\right.$ ) of early stage cervical cancer. Therefore, our study supports meta-analysis of previous literature with different ROI measurement techniques. Second, the use of less time-consuming $A D C_{\text {Mean }}$ measurement techniques in future studies is supported. $A D C_{\text {Mean }}$ showed to be a more independent predictor of parametrial involvement than tumor diameter and subjective assessment of parametrial invasion on T2W-MRI. In line with other studies, $A D C_{\text {Mean }}$ was able to predict lymph node metastases to some extent. Secondly, because we have demonstrated the independent potential of $A D C_{\text {Mean }}$ for the assessment of parametrial and lymph node involvement, $A D C_{\text {mean }}$ should be considered as a predictor in future predictive models for these adverse outcomes after surgery.

PART II. MR AND PET IMAGING FOR PREDICTING RESPONSE FOR LOCALLY

\section{ADVANCED CERVICAL CARCINOMA}

The aims of chapter $\mathbf{4}$ were to evaluate the ability of pelvic MRI to detect residual tumor after RT, to identify and validate objective imaging criteria predictive for residual tumor, and to assess their performance. It was shown that the use of objective MRI criteria improved the interobserver agreement and the diagnostic performance of the less experienced reader for assessment of residual disease after RT. The results obtained with the last brachy application were rather poor compared to the evaluation 3 months after RT. We also showed that the evaluation of MRIs obtained during RT could assist in the evaluation after treatment.

We therefore support the use of 'objective' MR criteria for the detection of residual tumor after RT with higher interobserver agreement compared to subjective evaluation; however, taking into account that the use of 'isointensity' on T2W-MRI as a standalone criterion performs only moderate-average. The combination of 'isointensity' with 'nodular shape' and 'irregular border' could improve the assessment of residual disease, with the condition that this regression model has to be validated.

Chapter 5 assessed if absolute tumor volume and tumor regression during radiotherapy could discriminate between patients with a complete response and those with a residual tumor after radiotherapy. Absolute volume measurements as well as volume regression at BCT appeared to be promising predictors of local residual disease. If future studies validate our results, volume regression might become an important predictor of response to CRT for locally advanced cervical cancer 
Chapter 6 shares our first experience with the PET-MRI in the evaluation of radiotherapy treatment to discriminate between patients with a complete local response and patients with local residual tumor. The addition of PET to MRI diminished both false positive and false negative MRI findings. Moreover, it was shown that the PET component was able to detect metastatic disease, significantly influencing the policy for these patients.

This second finding is important because approximately $50 \%$ of patients with local residual disease have remote metastases simultaneously. Future studies will show whether PET-MR proves to be capable of accurately assessing local residual cervical cancer while excluding metastatic disease.

Chapter 7, the general discussion, focused on the contribution of our findings to the existing literature, future perspectives, and directions for future research. 


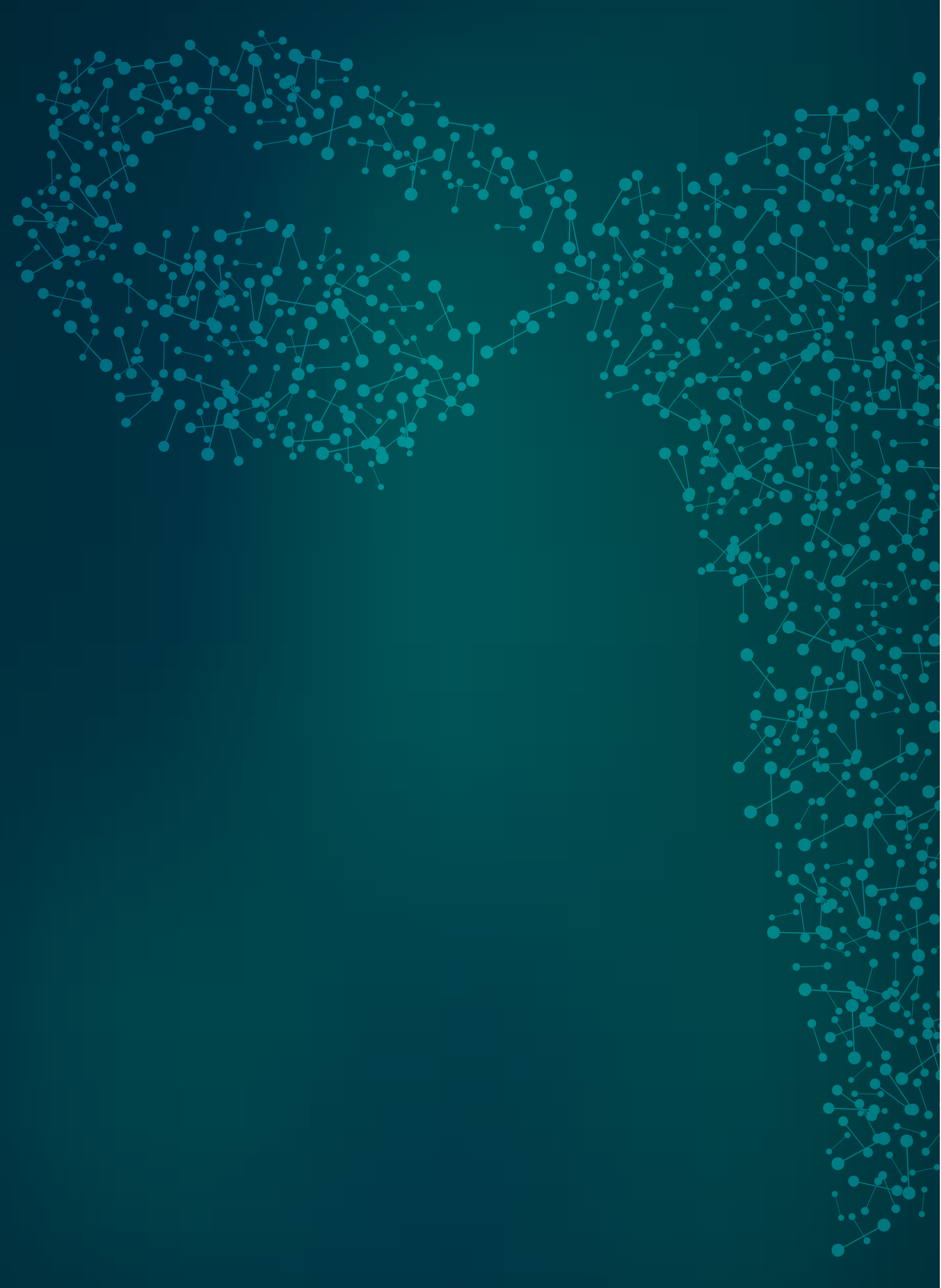

APPENDICES

VALORISATIE

ABBREVATIONS

PUBLICATIONS AND PRESENTATIONS

DANKWOORD

CURRICULUM VITAE 


\section{VALORISATIE}

In onze studies heeft de focus gelegen op het verbeteren van de stadiering van patiënten met cervixcarcinoom en het herkennen van patiënten met een incomplete reactie op chemoradiatie behandeling. De potentiële maatschappelijke en financiële waarden zullen in dit hoofdstuk uitgelicht worden.

\section{DE IMPACT VAN VERBETERING VAN DE STADIËRING}

In het vroeg stadium cervixcarcinoom kan een scala aan behandelingen worden aangeboden met bij elke behandeling specifieke voor en nadelen. Het stadium van de ziekte is in de regel leidend voor de te kiezen strategie, derhalve is correcte stadiëring essentieel. Dit is niet eenvoudig: het verschil tussen wel (stadium IIB) of geen (stadium I/IIA) parametrium invasie is niet altijd goed vast te stellen. Patiënten met parametrium invasie vastgesteld na chirurgie wordt geadviseerd om additioneel (chemo)radiatie te ondergaan om de kans op terugkeer van ziekte te verkleinen. Echter deze additionele (chemo)radiatie gaat gepaard met een hoger complicatierisico en meer bijwerkingen in vergelijking met patiënte die primair (chemo)radiotherapie hebben gekregen bij preoperatief vastgestelde parametrium invasie. Derhalve is het van belang dat de stadiering voor de operatie klopt. In het eerste gedeelte van het proefschrift hebben wij ons dan ook gericht op preoperatief accuraat voorspellen van parametrium invasie.

In hoofdstuk 2 laten wij zien dat in vergelijking met standaard MRI met de gefuseerde diffusie gewogen MRI gemiddeld 31\% vaker parametrium invasie wordt gevisualiseerd. Aangezien het voorkomen van parametrium invasie in onze populatie $12 \%$ is, zal over de gehele populatie $3,7 \%$ (31\% van $12,3 \%$ ) minder onverwachte postoperatieve parametrium invasie worden gezien. Dit omdat voor 3,7\% zal gekozen worden voor primaire chemoradiatie als therapie zonder voorafgaande radicale uterusextirpatie. De integratie van de in hoofdstuk 2 geïntroduceerde gefuseerde diffusie gewogen MRI voor de stadiëring van baarmoederhalskanker, zou een jaarlijkse kostenbesparing van grof berekend 150.000 euro kunnen opleveren. ${ }^{1}$ Hierbij is geen rekening gehouden met de indirecte medische kosten, directe niet-medische kosten en het toegenomen complicatierisico gerekend. Met betrekking tot het complicatierisico wordt een toename van $27 \%$ gerapporteerd indien primaire radiotherapie vergeleken wordt met chirurgie waarna aanvullende radiotherapie. Dit verschil wordt voornamelijk toegeschreven 
aan een toename van hydronefrose (5 versus 10\%) en van oedeem in de onderste extremiteit $\left(0,6\right.$ versus $11,1 \%$ ). . $^{(1)}$ Toename van oedeem resulteert in een afname van fysiek welbevinden, kwaliteit van leven en een toename van (revalidatie)kosten. ${ }^{(2,3)}$

Hoewel niet statistisch significant bewezen, wordt ook verwacht dat met diffusie gewogen MRI de specificiteit zal toenemen. In andere cohortstudies (specificiteit: 85$86 \%$ ) wordt een toename van de specificiteit met $9-14 \%$ gezien, overeenkomend met de door ons geobserveerde toename van 7-9\%. ${ }^{(4,5)}$ De toename in specificiteit zorgt ervoor dat patiënten niet ten onrechte worden gealloceerd naar chemoradiatie. Een buitenlandse studie laat reeds zien dat in de groep zonder nabehandeling chirurgie te prefereren is vanuit een sociaal en financieel oogpunt.(6) Een Nederlandse studie kon geen verschil in kwaliteit van leven aantonen maar suggereert wel een beter sociaal, seksueel en fysiek welbevinden in de chirurgie versus de chemoradiatie groep. ${ }^{(7)}$

Een kosteneffectiviteit studie in patiënten met een FIGO IB cervixcarcinoom laat zien dat chirurgie duurder is in vergelijking met (chemo)radiatie therapie ten faveure van een toename in de kwaliteit van leven. De extra kosten voor 1 extra 'quality-adjusted life year' (QALY) bedragen $400 € €^{(6)}$ Het financiële en sociale voordeel gold echter alleen voor de groep patiënten die na chirurgie geen aanvullende behandeling nodig hadden. Dit onderstreept het belang van een correcte stadiëring; voorkomen van patiënten die onnodig een operatie ontzegd wordt, zonder dat patiënten additionele therapie nodig hebben na chirurgie.

Kortom, de verwachting is dat de integratie van diffusie gewogen MRI in de dagelijkse praktijk een financieel voordeel zal opleveren met een afname van morbiditeit voor de patiënt.

Voortbordurend op de wens om morbiditeit te minimaliseren, tracht de SHAPE-trial minder radicale chirurgie uit te voeren in laag-risicopatiënten; simpele uterusextirpatie in plaats van radicale uterusextirpatie. ${ }^{(8)}$ Indien oncologische veiligheid wordt aangetoond zouden analoog aan deze studie onze resultaten gebruikt kunnen worden om meer patiënten te selecteren voor minder radicale chirurgie. Om zo een betere situatie voor de patiënt te creëren. Daarnaast is een simpele hysterectomie versus een radicale uterusextirpatie een financieel gezien meer aantrekkelijke oplossing met een kortere operatieduur, opnameduur en een sneller postoperatief herstel.
VERDERE MOGELIJKHEDEN TEN AANZIEN VAN DE VERBETERING VAN DE STADIËRING

In hoofdstuk 3 tonen wij de mogelijkheden van de gemiddelde Apparent Diffusion Coëfficiënt (ADC) gemeten op de preoperatieve MRI voor het voorspellen van parametrium invasie en lymfekliermetastasen. De integratie van meerdere risicofactoren zoals ADC voor het voorspellen van parametrium invasie, lymfekliermetastasen en de noodzaak tot additionele (chemo)radiatie na chirurgie lijkt onvermijdelijk. Toekomstige onderzoeken zouden de in hoofdstuk 2 en 3 opgedane kennis kunnen integreren met bekende individuele risicofactoren om tot een model te komen. Idealiter is dit een dynamisch model waarbij bijvoorbeeld 'machine based learning' en/of 'radiomics' worden gecombineerd met bekende risicofactoren. Individuele risico inschattingen zullen de gynaecoloog-oncoloog helpen om samen met de patiënt de optimale behandelstrategie te kiezen; het vermijden van chemoradiatie na chirurgie zonder patiënten onnodig een operatie te ontzeggen. Binnen deze toekomstvisie zullen patiënten-preferentie en kosten-baten studies onmisbaar zijn.

\section{DE (POTENTIËLE) IMPACT VAN HET VOORSPELLEN VAN HET PERSISTEREN VAN}

\section{CERVIXCARCINOOM NA CHEMORADIATIE}

In het $2^{\mathrm{e}}$ deel van het proefschrift probeerden we met diverse beeldvormende technieken lokaal persisteren van de ziekte aan te tonen zonder patiënten onnodig ongerust te maken. Indien onze prospectieve cohortonderzoeken dit bevestigen, zijn wij beter in staat om patiënten met restziekte te onderscheiden van patiënten met een complete reactie op de chemoradiatie doormiddel van volume metingen, MRI-criteria en PET-MR onderzoeken.

Meerdere studies hebben aangetoond dat het verrichten van chirurgie in een niet geselecteerde populatie enkele weken na radiotherapie niet resulteert in een toename van survival, ondanks een toename van de morbiditeit tot $25 \%$. Derhalve wordt door experts en richtlijnen geadviseerd om 2-3 maanden na chemoradiatie onderzoek en/ of beeldvorming te verrichten om de patiënten met significante lokale resttumor op te sporen. ${ }^{(9)}$ Patiënten met (geïsoleerde) resttumor krijgen radicale chirurgie aangeboden, zijnde de enige kans op langdurige overleving (30-40\%). Het is echter van cruciaal belang dat een test een hoge mate van accuratesse heeft om zo min mogelijk foutpositieven patiënten te opereren; radicale chirurgie na radiotherapie gaat gepaard met ernstige morbiditeit (tot $50 \%$ ) en mortaliteit (tot $2 \%$ ). ${ }^{(10}$

Hopelijk kunnen we in de toekomst de patiënten selecteren die baat hebben bij aanvullende chirurgische therapie met als gevolg een toename in overleving. In onze 
onderzoeken ligt de focus op het optimaliseren van de analyse van de beeldvormende technieken. Dit betekent echter dat er in de toekomst ook onderzoek zal moeten plaatsvinden om de kosteneffectiviteit te beoordelen. Het aantal (PET)-MRI ten opzichte van de overlevingswinst zal tegen elkaar moeten worden afgewogen.

In onze studies (hoofdstuk 4 en 5 opgeteld) werden 52 mensen onderzocht, waarvan $5,8 \%{ }^{33}$ in aanmerking kwam voor additionele chirurgie in verband met lokale rest ziekte. Dit komt overeen met de literatuur (6-7\%). ${ }^{(11)}$ Uit eerdere studies weten we dat $50 \%$ van de patiënten profijt heeft van de chirurgie, echter $50 \%$ recidiveert ongeacht de interventie. Derhalve zal bij $2,7 \%$ van de populatie terecht radicale chirurgie plaatsvinden als gevolg van de (PET)-MRI. Dientengevolge zal een minimum van 7,2 QALY's moeten worden behaald met het gebruik van MRI en een minimum van 12,2 QALY's per 100 patiënten met PET-MRI (vanwege de hogere kosten). ${ }^{2}$

Op basis van bovenstaande berekening blijkt de selectie van patiënten opnieuw cruciaal. Hoofdstuk 5 laat zien dat een eerste risicobeoordeling kan worden gemaakt op basis van reguliere MRI-scans verricht ten tijde van de brachytherapie. Bij ongeveer $70 \%$ van de populatie is er een aanzienlijke volume regressie te zien ten tijde van de eerste brachytherapie scan. Deze patiënten lijken een zeer kleine kans te hebben om geïsoleerde lokale rest ziekte te ontwikkelen na therapie; de beperkt beschikbare literatuur ondersteunt deze bevinding. ${ }^{(12,13)}$ Wanneer de prospectieve cohort data dit bevestigt, is het te verdedigen om de beeldvorming in de follow-up alleen in de resterende hoog-risicogroep te laten plaatsvinden. Op deze manier is het waarschijnlijker dat kosteneffectiviteit zal worden bereikt, terwijl tegelijkertijd de belastende onderzoeken voor $70 \%$ van de populatie worden gereduceerd.

\section{VERDERE MOGELIJKHEDEN TEN AANZIEN VAN DE VERBETERING VAN}

\section{BEELDVORMING NA CHEMORADIATIE}

Op basis van hoofdstuk 6 lijkt de PET-component (ten opzichte van de MRI) een toegevoegde waarde te hebben voor het beoordelen van de lokale tumorrespons na chemoradiatie. Bovendien zorgt de PET-component voor significante veranderingen in het beleid door detectie van simultane metastasen. Hopelijk kunnen toekomstige studies deze bevindingen bevestigen. De additionele waarde van diffusie gewogen MRI is niet onderzocht in deze pilotstudie. Gebaseerd op een recente andere studie

$2100 x$ kosten (PET)-MR + 5,8x kosten exenteratieve chirurgie $/ 20.000^{*}=$ minimaal aantal benodigde extra QALY's voor kostenefficiëntie. Kosten MRI: 316 euro, kosten PET: 994 euro, kosten chirurgie: 19430 euro. *Voor matschapelijk werantwoord geacht (Thok cont Pharmacoeconomics 2018) lijkt diffusie gewogen MRI wel de moeite waard om in de toekomst te onderzoeken. (14) Daarnaast wordt verwacht dat toekomstige ontwikkelingen zoals 'machine based learning' en 'radiomics' de mogelijkheden en nauwkeurigheid van de beeldvormende technieken naar een hoger niveau zullen tillen.

Ten slotte zal toekomstig onderzoek ook moeten kijken naar mogelijkheden om tijdens de therapie al voorspellingen te doen over het persisteren van de ziekte. Op basis van onze bevindingen in hoofdstuk 5 en recente literatuur, lijkt het de moeite waard om tijdens de therapie al dosisaanpassingen door te voeren. ${ }^{(13)}$ Vervolgstudies kunnen onderzoeken of het mogelijk is om tijdens de therapie te kiezen voor individualisatie van de beoogde dosis radiotherapie. Patiënten met een vermoeden op een slechte lokale respons zouden een hogere dosis kunnen krijgen met als gevolg een betere lokale controle maar ook met een toename van complicaties. Op basis van retrospectieve data zou een verhoging van de radiotherapiedosis met 5 gray bij patiënten met een slechte response (25\% volume reductie bij de eerste brachytherapie) kunnen zorgen voor een toename van $10 \%$ in lokale controle. ${ }^{(13)}$ Theoretisch gezien zou bij patiënten met een zeer goede initiële response juist de dosis verlaagd kunnen worden om het risico op complicaties te verminderen, dit zal echter nog prospectief onderzocht moeten worden. ${ }^{13}$

\section{CONCLUSIE}

Dit proefschrift ondersteunt de integratie van diffusie gewogen MRI voor de beoordeling van parametrium invasie bij het cervixcarcinoom. Daarnaast suggereren onze data het gebruik van ADC om de voorspelling van parametrium invasie verder te optimaliseren. Ten aanzien van het aantonen dan wel uitsluiten van lokale rest ziekte na chemoradiatie lijken volume metingen tijdens de radiotherapie, MRI-criteria en de toevoeging van $\mathrm{PET}$ veelbelovend.

De kennis die is opgedaan zal verder moeten worden uitgebreid, zodat de bevindingen daadwerkelijk invloed kunnen hebben op maatschappelijke en financiële domeinen, maar vooral de zorg voor de individuele patiënt kunnen verbeteren. 


\section{REFERENTIE}

1. Landoni F, Maneo A, Colombo A, Placa F, Milani R, Perego P, et al. Randomised study of radical surgery versus radiotherapy for stage Ib-Illa cervical cancer. Lancet (London, England). 1997;350(9077):535-40.

2. Kim SI, Lim MC, Lee JS, Lee Y, Park K, Joo J, et al. Impact of lower limb lymphedema on quality of life in gynecologic cancer survivors after pelvic lymph node dissection. Eur J Obstet Gynecol Reprod Biol. 2015;192:31-6.

3. Carter J, Huang HQ, Armer J, Carlson JW, Lockwood S, Nolte S, et al. GOG 244 - The LymphEdema and Gynecologic cancer (LEG) study: The association between the gynecologic cancer lymphedema questionnaire (GCLQ) and lymphedema of the lower extremity (LLE). Gynecologic oncology. 2019;155(3):452-60

4. Park JJ, Kim CK, Park SY, Park BK. Parametrial invasion in cervical cancer: fused T2-weighted imaging and high-b-value diffusion-weighted imaging with background body signal suppression at $3 \mathrm{~T}$. Radiology. 2015:274(3):734-41.

5. Qu JR, Qin L, Li X, Luo JP, Li J, Zhang HK, et al. Predicting Parametrial Invasion in Cervical Carcinoma (Stages IB1, IB2, and IIA): Diagnostic Accuracy of T2-Weighted Imaging Combined With DWI at 3 T. AJR Am J Roentgenol. 2018;210(3):677-84

6. Katanyoo K, Praditsitthikorn N, Tangjitgamol S, Manusirivithaya S, Supawattanabodee B. Cost-utility analysis of treatments for stage IB cervical cancer. Journal of gynecologic oncology. 2014;25(2):97-104

7. Derks M, van Lonkhuijzen LRCW, Bakker RM, Stiggelbout AM, de Kroon CD, Westerveld H, et al. Long-Term Morbidity and Quality of Life in Cervical Cancer Survivors: A Multicenter Comparison Between Surgery and Radiotherapy as Primary Treatment. Int J Gynecol Cancer. 2017;27(2):350-6.

8. Group CCT. Radical Versus simple Hysterectomy and Pelvic Node Dissection With Low-risk Early Stage Caervical Cancer (SHAPE). ClinicalTrialsgov. 2012.

9. Cibula D, Potter R, Planchamp F, Avall-Lundqvist E, Fischerova D, Haie Meder C, et al. The European Society of Gynaecological Oncology/European Society for Radiotherapy and Oncology/European Society of Pathology guidelines for the management of patients with cervical cancer. Radiother Oncol. 2018:127(3):404-16.

10. Matsuo K, Mandelbaum RS, Adams CL, Roman LD, Wright JD. Performance and outcome of pelvic exenteration for gynecologic malignancies: A population-based study. Gynecol Oncol. 2019;153(2):368-75.

11. Schmid M. Local failures after radiochemotherapy and MR-image-guided brachytherapy in cervical cancer patients. . Radiother Oncol 2016;2017;123:S26.

12. Wang JZ, Mayr NA, Zhang D, Li K, Grecula JC, Montebello JF, et al. Sequential magnetic resonance imaging of cervical cancer: the predictive value of absolute tumor volume and regression ratio measured before, during, and after radiation therapy. Cancer. 2010;116(21):5093-101.

13. Schernberg A, Bockel S, Annede P, Fumagalli I, Escande A, Mignot F, et al. Tumor Shrinkage During Chemoradiation in Locally Advanced Cervical Cancer Patients: Prognostic Significance, and Impact for Image-Guided Adaptive Brachytherapy. Int J Radiat Oncol Biol Phys. 2018;102(2):362-72.
14. Thomeer MG, Vandecaveye V, Braun L, Mayer F, Franckena-Schouten M, de Boer P, et al. Evaluation of T2-W MR imaging and diffusion-weighted imaging for the early post-treatment local response assessment of patients treated conservatively for cervical cancer: a multicentre study. Eur Radiol. 2019;29(1):309-18 


\section{ABBREVATIONS}

ADC:

AUC:

$\mathrm{BCT}$.

CEA:

CRT:

CT:

CT:

DICOM:

DWI:

EBRT:

ESGO:

ETL:

FDG-PET:

FIGO:

FOV:

GEC-ESTRO:

Gy:

HR-CTV:

$\mathrm{HT}$ :

IGABT:

IRB:

IR-CTV:

凶:

LVSI:

MRI:

NCCN:

NSA:

OR:

PA:

PACS:

PET:

ROI:

Apparent Diffusion Coefficient

Area Under The Receiver Operating Characteristics Curve

Brachytherapy

Carcinoembryonic antigen

Chemoradiation treatment

Chemotherapy

Computer Tomography

Digital Imaging and Communications in Medicine

Diffusion Weighted Imaging

External Beam Radiotherapy

European Society of Gynecologic Oncology

Echo Train Length

Fluorodeoxyglucose-Positron Emission Tomography

international Federation of Gynecology and Obstetrics

Field of View

The Groupe Européen de Curiethérapie (GEC) and the European SocieTy for Radiotherapy \& Oncology (ESTRO)

Gray

High risk clinical target volume

Hyperthermia

Image guided adaptive brachytherapy

Institutional Review Board

intermediate risk clinical target volume

Kappa

Lymph vascular space invasion

Magnetic Resonance Imaging

National Comprehensive Cancer Network

Numbers of signals averaged

Odds ratios

Pathology

Picture Archiving and Communication System

Positron Emission Tomography

Region Of Interest

Radiation treatment

Squamous-cell carcinoma antigen

Standard Uptake Value 
Echo time

Repetition time

$\begin{array}{ll}\text { T1W: } & \text { T1 weighted } \\ \text { T2W: } & \text { T2 weighted }\end{array}$

2D: Two-dimensional

3D: Three-dimensional

1.5T: $\quad 1.5$ Tesla

3.0T: $\quad 3.0$ Tesla 


\section{PUBLICATIONS AND PRESENTATIONS}

\section{PUBLICATIONS}

Mongula JE, Bakers F, Kruitwagen RFPM, Slangen BFM et al. Assessment of parametrial invasion of cervical carcinoma, the role of T2-weighted MRI and diffusion weighted imaging with or without fusion. Clinical Radiology. 2019

Mongula JE, Bakers F, Slangen BFM, Kruitwagen RFPM, Mihl C et al. Evaluation of various Apparent Diffusion Coefficient measurement techniques in pre-operative of early cervical cancer staging. European Journal of Radiology. 2019

Mongula JE, Slangen BFM, Kruitwagen RFPM et al. 18F-FDG Positron emission tomography - Magnetic resonance imaging (PET-MRI) for response assessment after radiotherapy for cervical carcinoma: an initial experience. EJNMMI Research. 2018

Mongula JE, Slangen BFM, Kruitwagen RFPM, Beets-Tan RGH et al. Consecutive magnetic resonance imaging during brachytherapy for cervical carcinoma: predictive value of volume measurements with respect to persistent disease and prognosis. Radiation Oncology. 2015

Mongula JE, Slangen BFM, Kruitwagen RFPM, Beets-Tan RGH et al. Predictive criteria for MRI-based evaluation of response both during and after radiotherapy for cervical cancer. Journal of Contemporary Brachytherapy. 2016

Mongula JE, Frenken M, Porath M, Goossens S et al. COVID-19 during pregnancy: fetal distress, placental pathology and disseminated intravascular coagulation. Submitted

van de Berg K, Mongula JE, Slangen BFM et al. The predictive value of squamous cell carcinoma antigen in cervical cancer. Submitted

Mongula JE, Brueren M. Antidepressiva. Huisarts en Wetenschap. 2010

Mongula JE, van Koeverden S, Aarts A. Hinderlijke zwelling ter hoogte van het linker labium. Medisch Contact. 2020 


\section{PRESENTATIONS}

Mongula JE et al. Response evaluatie na chemoradiatie bij het cervixcarcinoom: de waarde van MRI. Regionale refereeravond Gynaecologie, 2013, Maastricht (oral presentation)

Mongula JE et al. Retrospective response evaluation of cervical carcinoma: the value of MRI. GROW School for Oncology and Developmental Biology (GROW) meeting. 2011, Maastricht (oral presentation)

Mongula JE et al. DW-MRI of cervical carcinoma, ADC measurement techniques related to parametrial and lymph node involvement. 20th European Society of Gyneacologic Oncology Meeting, 2017, Wenen (poster)

Mongula JE et al. The additional role of MRI and DWI for the assessment of parametrial invasion of cervical carcinoma. 20 th European Society of Gyneacologic Oncology Meeting, 2017, Wenen (poster)

Mongula JE et al. PET-MRI for response assessment after radiotherapy for cervical carcinoma: an initial experience. $16^{\text {th }}$ International meeting of the Gynecologic Cancer Society, 2016, Lissabon (poster)

Mongula JE et al. MRI for predicting residual disease after radiotherapy in locally advanced cervical cancer. $17^{\text {th }}$ International meeting of the European Society of Gynecologic Oncology, 2011, Milaan (poster)

Mongula JE et al. Magnetic resonance imaging for predicting residual disease after radiotherapy in locally advanced cervical cancer. GROW, GROW Science day, 2011, Maastricht (poster)

Mongula JE et al. Evaluation of cervical cancer post-radiotherapy: differentiation of persistent tumor versus radiation induced fibrosis. Clinical Investigator Science Symposium, 2011, Maastricht (poster) 


\section{DANKWOORD}

\section{'WIE IN HET DAL BLIJFT ZIET NOOIT DE ANDERE KANT VAN DE BERG'.}

Dit proefschrift was die berg en het dal ligt achter ons, na een lange en bij tijden slopende klim. Dank aan iedereen die mij heeft geholpen en weten te motiveren om de berg te blijven beklimmen. Dit proefschrift was er niet gekomen zonder jullie hulp. Een aantal van jullie wil ik graag in het bijzonder bedanken.

Bijzondere dank gaat uit naar mijn copromotor dr. Brigitte Slangen. Zonder jou was dit project er niet gekomen. Jaren geleden op het door de universiteit georganiseerde afstudeerfeestje van de A-KO kwam je al langs om mij te feliciteren. Je kwam speciaal voor mij of kwam je toch gewoon voor het feestje? Ik kwam er namelijk al snel achter dat jij zelden een feestje overslaat. Op de werkvloer had je een kritische blik, dit koste me soms veel tijd maar achteraf was ik er erg blij mee. Je stond en staat altijd voor me klaar en dit waardeer ik enorm. Het hoeft bij jou niet altijd werkgerelateerd te zijn, er is altijd ruimte om het over 'de andere belangrijke zaken' in het leven te hebben. Ik heb een vermoeden dat onze reis nog niet klaar is, de onderzoeksprojecten lopen door en dat er nog maar velen mogen volgen. Dank voor je steun en vertrouwen.

Beste professor Kruitwagen, beste Roy. Bedankt voor uw vertrouwen in een goede afloop van het promotietraject. Na een eerste drankje, op het terras tegenover de Duomo in Milaan, moest ik het beeld van mijn promotor toch bijstellen. Als laatste jaars geneeskunde student was ik toch wat zenuwachtig als ik bij $u$ op kantoor mocht verschijnen. Dit bleek nergens voor nodig. In al die jaren bent u toegankelijk, behulpzaam en bovenal slagvaardig geweest. Zo kreeg ik manuscripten nagenoeg altijd binnen 1 week terug en wanneer ik ook maar enigszins twijfelde over de voortgang van het project, wist u mij te motiveren. Bedankt voor alle ondersteuning in het onderzoek en de opleiding.

Beste dr. Mihl, beste Casper. Ik weet nog dat ik je leerde kennen, je was enorm enthousiast en had veel zin in het project. We begonnen met het beoordelen van de eerste scans, echter de eerste 30 minuten scoorde we geen enkele MRI-scan. Het was namelijk nog belangrijker dat ik de juiste carbon velgen ging kopen voor mijn racefiets...... Daarnaast kwam Down The Rabbit Hole eraan, dus dat moest natuurlijk ook even besproken worden. Wij bleken veel gemeenschappelijke interesses te hebben. Het was dan ook altijd gezellig maar er werd ook veel werk verzet. Bedankt voor alle tijd die je in ons project hebt gestoken en die je in de vervolgprojecten blijt steken. Dit was je eerste copromotor schap, maar vast niet de laatste. 
Voorzitter en leden van de promotiecommissie: prof. dr. J.E. Wildberger, prof. dr. R.L.M. Bekkers, prof. dr. V. Vandecaveye, dr. S. Lambrechts en dr. R.A. Nout, bedankt voor het beoordelen van mijn proefschrift.

Beste Frans Bakers, bedankt voor alle tijd die je hebt gestoken in het onderzoeksproject. Het scoren van alle MRI's heeft de basis voor een mooi proefschrift opgeleverd. De vervolgstudies zijn goed onderweg. Ik ben ervan overtuigd dat wij hier met zijn allen, nog mooie resultaten uit gaan halen. Ik kijk uit naar jouw proefschrift!

Beste Sander van Kuijk, bedankt voor alle statistische hulp, onze discussie leverde naast een beter artikel, vaak ook nieuwe onderzoeksideeën op. Als mijn ideeën op zijn, kom ik weer met je discussiëren.

Overige coauteurs (Doenja, Ludy, Toon, Roy (Vliegen), Francesco, Stefan, Arnold en Regina), bedankt voor jullie input in alle afzonderlijke artikelen.

Beste gynaecologen van het MUMC+, VieCuri en MMC, bedankt voor de fijne jaren. Bedankt voor de tijd in de opleiding die ik heb gekregen om het proefschrift te verwezenlijken. Christine Willekes, wij kennen elkaar al 12 jaar. Jij zette mij op het juiste 'gynaecologische' spoor in de Arts-Klinisch Onderzoeker master en adviseerde me vooral met Brigitte te gaan praten. Kijk wat ervan is gekomen...

Opleiders (Roy Kruitwagen, Marc Spaanderman, Toon van Gorp, Iris van Gestel en recent Jacques Maas) bedankt voor de ondersteuning om dit project tijdens de opleiding af te ronden.

De overige gynaecoloog-oncologen van het MUMC+: Sandrijne, Peggy en Erica. Hartelijk dank voor de fijne tijd die ik de afgelopen jaren bij jullie heb gehad. Dank ook voor de momenten om te sparren over het onderzoek en voor de bijdrage in mijn opleiding tot gynaecoloog. Hopelijk komen wij elkaar in de toekomst nog regelmatig tegen.

Beste collega AIOS van het MUMC+, VieCuri en recent MMC, bedankt voor de fijne opleidingstijd. Fijn dat wij als groep er voor elkaar zijn en elkaar helpen waar nodig.

Vrienden bedankt

De (echte) Nijmeegse fietsclub: bedankt voor het bieden van een luisterend oor tijdens de mooie fietstochten. De mogelijkheid tot ontspanning in combinatie met de sociale interactie hebben een significante bijdrage geleverd aan dit project. Ik hoop dat ik nog regelmatig mag afzien in jullie wiel.
De bonobo's: onze paden zijn wat meer uit elkaar gelopen, echter de band die wij hebben opgebouwd in onze studententijd, blijft bestaan. In het bijzonder Wouter, Hugo, Marcel en Jasper bedankt

Tom Schouwenaar, of het nu op de fiets, de kroeg, het festival of de wijnproeverij is, je bent er te vinden. Je vermogen om het overal gezellig te maken maar ook tijd te hebben om diepgaande gesprekken te voeren, waardeer ik enorm. Helaas ben je voor Feyenoord en ken je de spelregels van Kolonisten van Catan niet, maar desondanks ben je nu 10 jaar later een van mijn meest gewaardeerde vrienden en mijn paranimf. Dank voor beiden! Met Catelijne aan jouw zijde is de vriendschap tussen ons 4'en en recent 6'en (Moos en Charlotte) extra speciaal.

Bart Willem Oudelaar, je leeft volgens je eigen motto: 'You snooze, you loose'. Je tracht het maximale uit het leven te halen of dit nu sociaal, sportief of werk gerelateerd is. Misschien lijken we daarin wel op elkaar en kunnen we daarom zo goed met elkaar opschieten. Als er iets belangrijks in mijn leven gebeurt voel ik vaak de behoefte om dit met jou te bespreken. Een teken van echte vriendschap. Ik ben dan ook blij dat je mijn paranimf wil zijn

Lieve Familie, dank jullie voor alle steun, gezelligheid en motivatie. Daryll bedankt dat je mijn broertje bent. Fijn dat je 'relatief' ver van de medische wereld staat. Als wij samen zijn, staan hierdoor alle werkgerelateerde 'issues' echt op de achtergrond en hebben wij hele gezellige avonden. Vroeger met bier of whisky in de kroeg, tegenwoordig met wijn in een goed restaurant. Dat er nog maar veel restaurants mogen volgen. Beste Dolf en Renate, bedankt voor alle hulp rondom dit project, het heeft zeker bijgedragen. Beste Dolf, enorm respect voor hoe jij omgaat met de Parkinson. Je gaat (nagenoeg) onverstoord door en klaagt er eigenlijk nooit over. Renate, extra bedankt voor het vermaken van Moos en de input op fotografisch/design vlak. Je hebt er oog voor. Een beetje vreemd vind ik het dan ook dat je 'design landgoed Prijs' styled met tweedehands prullaria uit Tongeren en kringloopwinkels.....

Lieve pap en mam, bedankt voor jullie onvoorwaardelijke steun en belangstelling (ondanks dat jullie bij tijd en wijlen geen idee hadden wat ik aan het doen was). Maar bovenal bedankt voor de mogelijkheden en vrijheden die jullie mij in het leven hebben geboden. Wat ik ook ging doen, ik werd altijd volledig gesteund met de enige voorwaarde dat ik iets deed waar ik gelukkig van werd. Ik hoop hetzelfde voor Moos te kunnen betekenen.

Daarnaast natuurlijk ook bedankt voor alle logistieke hulp zodat ik tijd vrij kon maken voor dit project; van oppassen op Moos, helpen met het opknappen van het huis tot aan lekkere Indische maaltijden. Dat er daar nog maar velen van mogen volgen. 
Lieve Moos, je bent nieuw in mijn leven, je kunt dit nog niet eens lezen. Maar ook jij hebt je aandeel in dit project. Je eeuwige enthousiasme, lach en gezelligheid maken het leven leuker. Je lijkt wel een tovenaar; alle zorgen of frustraties laat je als sneeuw voor de zon verdwijnen. Zoals jij er voor mij bent, zal ik er ook voor jou zijn.

En nu de belangrijkste, Jolijn. Ik heb je moeten beloven het kort te houden.

Ondanks dat jezelf niet bepaald gek op onderzoek bent, heb je mij altijd volledig ondersteund. Zowel in dit project als in alle andere aspecten van het leven. Wij zijn al zo lang samen dat ik oprecht kan zeggen dat ik hier niet zou zijn aangekomen zonder jou. Je zorgt ervoor dat ik op de rails blijf met je geduld, inlevingsvermogen en liefde. Je hebt je af en toe moeten wegcijferen en mij gefaciliteerd om dit project mogelijk te maken. Heel veel dank hiervoor, je bent de beste, ik hou van je! 


\section{CURRICULUM VITAE}

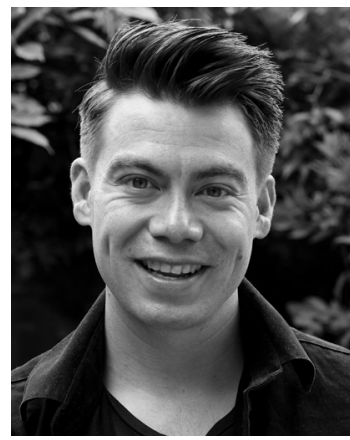

Jordy Mongula werd geboren in Zevenaar op 12 augustus 1986. Zijn atheneumdiploma werd in 2004 behaald aan het Candea College te Duiven. Hij startte direct daarna met de bachelor Biomedische Technologie aan de Technische Universiteit te Enschede. Na de bachelor werd hij aangenomen voor de master tot Arts-Klinisch Onderzoeker in Maastricht. Tijdens zijn wetenschappelijke stage in het laatste jaar van de opleidingen werden beeldvormende technieken gecombineerd met de gynaecologie. De basis voor dit proefschrift werd hier gelegd. Onder leiding van prof. Roy Kruitwagen en dr. Brigitte Slangen werd een promotie traject opgestart waarvan de belangrijkste bevindingen in dit proefschrift beschreven worden. In oktober 2011 behaalde hij zijn artsexamen en werkte hij als arts-assistent niet in opleiding in het MUMC+ te Maastricht en Canisius Wilhelmina Ziekenhuis te Nijmegen. Vanaf medio 2014 is hij in opleiding tot gynaecoloog. De basisopleiding werd gevolgd in het MUMC+ te Maastricht en VieCuri te Venlo. De differentiatie gynaecologische oncologie werd in het MUMC+ en Radboud UMC te Nijmegen gedaan. Op dit moment werkt hij in het Maxima Medisch Centrum te Veldhoven, in het laatste deel van zijn opleiding differentieert hij in de minimaal invasieve chirurgie. 


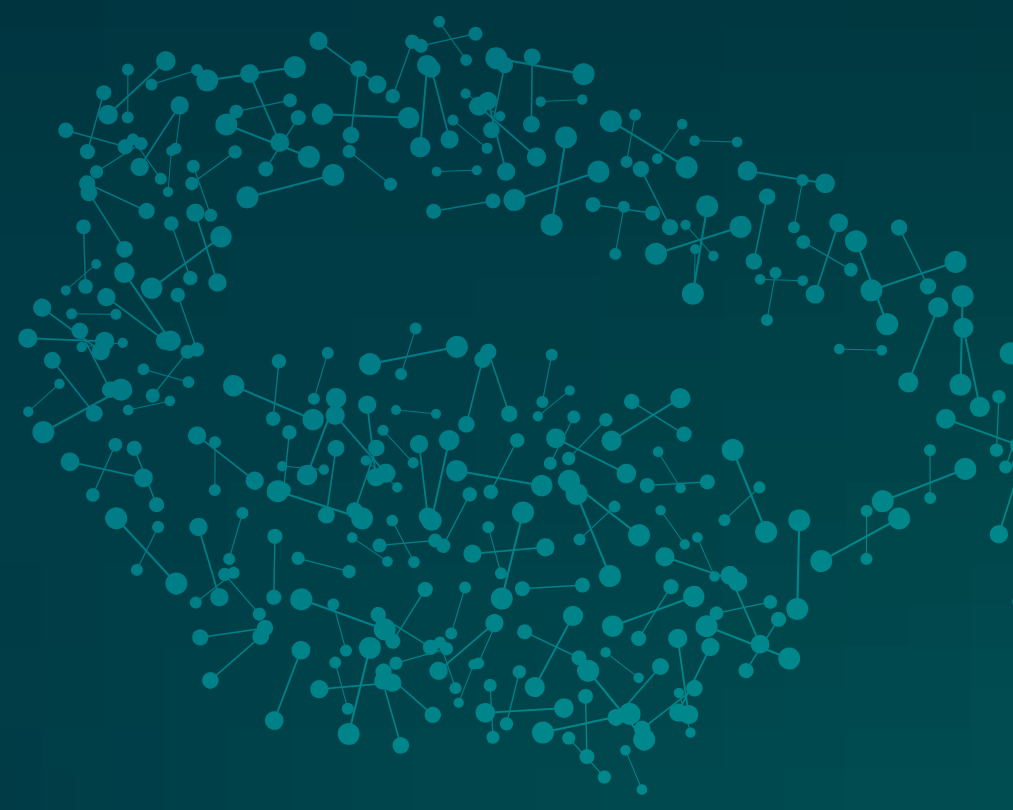

